\title{
Mapped finite element methods: High-order approximations of problems on domains with cracks and corners
}

\author{
Maurizio M. Chiaramonte ${ }^{1,2, *, \dagger(D), ~ Y o n g x i n g ~ S h e n ~}{ }^{3, *}, \dagger$ (D) and Adrian J. Lew ${ }^{2, *, \dagger}$ \\ ${ }^{1}$ Department of Civil and Environmental Engineering, Princeton University, Princeton, NJ 08544, USA \\ ${ }^{2}$ Department of Mechanical Engineering, Stanford University, Stanford, CA 94305, USA \\ ${ }^{3}$ State Key Laboratory of Metal Matrix Composites, University of Michigan-Shanghai Jiao Tong University Joint \\ Institute, Shanghai Jiao Tong University, Shanghai, 200240, China
}

\begin{abstract}
SUMMARY
Linear elasticity problems posed on cracked domains, or domains with re-entrant corners, yield singular solutions that deteriorate the optimality of convergence of finite element methods. In this work, we propose an optimally convergent finite element method for this class of problems. The method is based on approximating a much smoother function obtained by locally reparameterizing the solution around the singularities. This reparameterized solution can be approximated using standard finite element procedures yielding optimal convergence rates for any order of interpolating polynomials, without additional degrees of freedom or special shape functions. Hence, the method provides optimally convergent solutions for the same computational complexity of standard finite element methods. Furthermore, the sparsity and the conditioning of the resulting system is preserved. The method handles body forces and crack-face tractions, as well as multiple crack tips and re-entrant corners. The advantages of the method are showcased for four different problems: a straight crack with loaded faces, a circular arc crack, an L-shaped domain undergoing anti-plane deformation, and lastly a crack along a bimaterial interface. Optimality in convergence is observed for all the examples. A proof of optimal convergence is accomplished mainly by proving the regularity of the reparameterized solution. Copyright (C) 2016 John Wiley \& Sons, Ltd.
\end{abstract}

Received 5 April 2016; Revised 23 November 2016; Accepted 24 November 2016

KEY WORDS: solid; crack problem; re-entrant corner; singularity

\section{INTRODUCTION}

In elliptic boundary value problems, such as linear elasticity and the Poisson problem, which can describe steady-state heat conduction, steady-state diffusion, or electrostatics, singularities in the solution may arise from non-smooth boundaries, discontinuous coefficients, or abrupt changes in boundary conditions. Problems with cracked domains, or those containing re-entrant corners, fall in the first category. The singularity of the solutions to this class of problems plagues the accuracy and convergence rate of standard (Lagrange) finite element methods. Concretely, finite element approximations of linear elasticity solutions in cracked domains converge in $H^{1}$ with order $1 / 2$, regardless of the order of interpolating polynomials in each element. To alleviate the shortcomings of standard finite element methods, several techniques have been proposed to address these singularities. These often provide a more accurate solution, but very few of them are capable of obtaining optimal rates of convergence for an arbitrary order of interpolating polynomials, which, as we show here, substantially reduce the computational time for a given desired accuracy.

\footnotetext{
*Correspondence to: Maurizio M. Chiaramonte, Department of Civil and Environmental Engineering, Princeton University, Princeton, NJ 08544, USA; Yongxing Shen, University of Michigan-Shanghai Jiao Tong University Joint Institute, Shanghai Jiao Tong University, Shanghai, China; Adrian J. Lew, Department of Mechanical Engineering, Stanford University, Stanford, CA 94305, USA.

†Email: chiaramonte@ princeton.edu; yongxing.shen@sjtu.edu.cn; lewa@stanford.edu
} 
Overall speaking, the classes of finite element methods capable of achieving better accuracy than standard finite element methods at a given computational cost for crack and re-entrant corner problems can be arguably classified into four categories: A family of methods that enriches the finite dimensional space with additional basis functions, one that takes advantage of adaptive refinement of $h$ - and/or $p$-type, another that employs special singular elements, and lastly a family of methods, which is closely related to this work, that exploits mapping techniques to approximate a smoother function obtained from carefully mapping the singular solution. In the following paragraphs, we review the efforts invested in developing the aforementioned families of finite element methods.

Possibly, the most common methodology to enhance the accuracy of the solution and to achieve optimal convergence rates is through the addition of special basis functions to the finite element space. The work found in [1] was the precursor of the methodology, and more recently, [2-5] have re-energized this idea by including special basis functions exploiting the framework of the partition of unity. Within this framework, the methods proposed in [6-9] are among the early contributions that could achieve second-order convergence in the $L_{2}$-norm of the displacement. The literature on the topic is vast, and we refer the reader interested in a thorough review to [10,11].

In [12] and [13], the authors independently proposed a technique to construct eight-node quadrilateral elements whose shape functions resemble the $\sqrt{r}$ singularity encountered in linear elastic fracture mechanics, where $r$ denotes the distance to the crack tip. By placing the mid-side nodes at the quarter points, the mapped shape functions along the element edges exactly capture the radial singularity. Later, the method was improved in [14] where one of the element edges is collapsed to a single point so that the mapped shape functions capture the singularity in the interior of the element as well. This idea of edge collapse appeared in an earlier contribution [15] for the case of four-node quadrilateral elements. Although such degenerate elements constructed through singular isoparametric maps greatly enhance the accuracy of the solution, the convergence rates remain sub-optimal.

Another class of methods employs adaptive mesh refinement in the proximity of the singularity to enhance the convergence of the solution. Such specially designed graded meshes $[16,17]$ or adaptively determined polynomial order [18] can be shown to yield optimally convergent or otherwise much more accurate solutions.

Before we introduce the last class of methods, we discuss a different perspective on how to deal with singularities. Consider the singular solution $\boldsymbol{u}$ for an elasticity problem on a cracked domain $\Omega$, and define a function $\hat{\boldsymbol{u}}:=\boldsymbol{u} \circ \gamma$ on $\hat{\Omega}=\gamma^{-1}(\Omega)$, the result of composing $\boldsymbol{u}$ with a mapping $\gamma: \hat{\Omega} \rightarrow \Omega$. If, with a priori knowledge of the singularity, it were possible to construct a mapping $\gamma$ such that $\hat{\boldsymbol{u}}$ were much smoother that $\boldsymbol{u}$, then one could shift the focus to constructing approximations to $\hat{\boldsymbol{u}}$ instead of to $\boldsymbol{u}$. We show here that this is in fact possible. Why is approximating $\hat{\boldsymbol{u}}$ different than approximating $\boldsymbol{u}$ ? Because under very general conditions, $\hat{\boldsymbol{u}}$ is very smooth, and hence, standard finite element methods and quasiuniform family of meshes over $\hat{\Omega}$ can be used to approximate it with orders of convergence bounded only by the smoothness of $\hat{\boldsymbol{u}}$. This higher order of approximation is not lost when composing the approximation of $\hat{\boldsymbol{u}}$ with $\boldsymbol{\gamma}$ to construct an approximation of $\boldsymbol{u}$. This approach to deal with singularities is the core of the method we introduce here.

Ideas close to what was described earlier have been explored in the literature. What distinguishes our method from earlier work is that, by constructing a mapping $\gamma$ completely independent of the domain discretization, we create an entirely different problem from the original one, which we then proceed to discretize. This view is in contrast with the use of mapping techniques to effectively generate special shape functions resulting from map composition. Reformulating the problem entirely, alongside specially constructed mappings $\gamma$, circumvents many of the limitations of earlier works where the cumbersome meshing required, the limited capabilities in handling complex crack geometries (including multiple cracks and cracks arbitrarily close to domain features), alongside the lack of applications to the more commonly employed $h$-version of finite element methods, have likely prevented this type of approach from being widely adopted. To further elaborate on this, we briefly review previous effort expenditures and later highlight the novelties of our approach.

The origin of this class of methods is the work found in [19], where, through conformal mappings and a careful construction of the discretization of the parametric domain $\hat{\Omega}$, the authors obtained better approximations to the straight crack problem. Albeit their approach proved innovative, the method, which the authors referred to as the mapped elements method, suffered several limitations. 
In fact only a limited family of crack geometries were dealt with (only straight cracks), the mesh generation process was rather cumbersome, and the non-local mapping limited the applicability of the method to single cracks in simple domains. At a later time, one of the authors proposed [20] a similar technique that employs mappings to generate singular crack tip elements. Analogous to the work found in [12-14], such singular elements greatly enhance the accuracy of the solution, but the convergence rates remain sub-optimal.

In [21], the authors exploited the same underlying idea and named their approach the method of auxiliary mapping. Their method was primarily targeted to $p$-finite elements [22] and $h p$-finite elements. Similarly to [19], they reparameterized the solution restricted to a circular sector around the singularity and separately meshed this sector and the remaining part of the computational domain. The discretized circular sector was then mapped with the inverse mapping to the parametric domain (the domain over which the singularity is eliminated), and then, standard polynomial shape functions were built over this parametric domain. This interwoven nature of the mapping and the domain discretization render the meshing procedure restrictive and cumbersome: The approximation functions near the boundary between the circular sector and the remaining region have to be constructed using the blending mapping technique [23] to ensure conformity. With such construction, for the $p$-and $h p$-version of finite elements, they showed exponential rates of convergence. The method was later extended to address boundary singularities for a larger family of elliptic operators in [24], elasticity problems in [25], and three-dimensional domains in [26]. Recently, it was exploited in the context of isogeometric finite element methods [27].

We introduce here a method targeted to $h$-finite elements of arbitrary order but that should also work with $p$-finite elements, where the novelty lies in the fact that we construct an entirely new problem via a mapping that is independent of the choice of the numerical method and spatial discretization. Hence, standard numerical methods of any order could be adopted to approximate this new problem. Additional novel features of the method consist of

- The mappings are local, allowing the handling in a trivial manner of multiple singularities, and of singularities at interfaces or domain boundaries.

- The construction of high-order approximations significantly reduces the computational time needed to obtain numerical solutions with a given error, as we show here.

- A large family of mappings are proposed [not a single way of constructing a smooth solution, cf. (9)]. We provide precise conditions on the minimum power of the asymptotic behavior of the mapping near the singularity to sufficiently smoothen it to obtain optimal rates of convergence for a given order of the $h$-version of finite element method.

- The family of mappings contain many mappings that are $C^{\infty}(\Omega)$. One of them is adopted here for the numerical examples.

- The asymptotic behavior of the particular mapping we adopt here is such that we do not need to a priori know either the asymptotic expression or even the precise power of the singularity, being that of a corner, a crack, or a crack at an interface, to recover optimal order of convergence of the method.

- Both the enhanced regularity of the mapped solution and the optimality of the convergence rate of the resulting method are proved.

We highlight that the computational cost of the proposed method for a given number of degrees of freedom is the same as that of standard finite element methods, no ill-conditioning issues arise, several types of singularities can be successfully dealt with (including oscillatory ones), the method is trivially implementable, and moving singularities are easily handled (e.g., propagating cracks and moving dislocations).

Although we refer to this approach at dealing with singularities as a 'method', in reality, it is nothing but a reformulation of the problem statement such that the reformulated problem can be easily solved using standard methods over $\hat{\Omega}$. In particular, in the case of adopting finite element methods for this last approximation, pushing forward the shape functions from $\hat{\Omega}$ to $\Omega$ effectively builds a non-standard finite element space over $\Omega$. Motivated by this observation, and in line with what was originally proposed in [19], we will refer to our method with the acronym MFEM, for mapped finite element method. 
The paper is organized as follows. In Section 2, we provide a bird's-eye view of the method. We save its details for Section 3 where we provide a self-contained summary of it for the reader less interested in the detailed analysis. In Section 4, we use numerical examples to showcase the convergence properties of the method. Then, in Section 5, we analyze the method, showing the enhanced regularity of $\hat{\boldsymbol{u}}$ and the optimal convergence rates at which $\hat{\boldsymbol{u}}$ and $\boldsymbol{u}$ are approximated.

We conclude the introduction with some remarks on notation. We denote by $H^{k}\left(\Omega, \mathbb{R}^{d}\right)$ the Sobolev space $W^{k, 2}\left(\Omega, \mathbb{R}^{d}\right)$ and we let $\|\square\|_{k, \Omega}$ and $|\square|_{k, \Omega}$ denote the Sobolev norm and seminorm, respectively, over $\Omega$. We let $\nabla \cdot$ and $\nabla$ denote the divergence and gradient operators, respectively. We reserve the symbol $\boldsymbol{n}$ to denote outward normals to boundaries. We will denote by $\mathbf{1}:=\delta_{i j} \mathbf{e}_{i} \otimes \mathbf{e}_{j}$ the second-order identity tensor and by

$$
\mathbb{I}=\frac{1}{2}\left(\delta_{i k} \delta_{j l}+\delta_{i l} \delta_{j k}\right) \mathbf{e}_{i} \otimes \mathbf{e}_{j} \otimes \mathbf{e}_{k} \otimes \mathbf{e}_{l}
$$

the fourth-order identity tensor on symmetric second-order tensors, where $\left\{\mathbf{e}_{i}\right\}$ is an orthonormal basis.

\section{OVERVIEW OF THE METHOD}

We begin with a brief overview of the key ideas behind the method. Details of the formulation and the analysis are saved for Sections 3 and 5, respectively.

\subsection{The challenge}

Non-smooth boundaries yield singular solutions to certain elliptic boundary value problems such as linear elasticity. In this work, we consider a class of problems whose solution can be expressed as the sum of a singular part and a smooth part. For example, in a domain $\Omega$ with a re-entrant corner of angle $\Theta>\pi$ (Figure 1), the solution of the elastic field $u: \Omega \rightarrow \mathbb{R}^{2}$ can be written as the sum of singular functions, $\left\{\boldsymbol{f}_{i}\right\}$, plus a smooth part $\boldsymbol{u}_{R}$ such as

$$
\boldsymbol{u}=\sum_{i=1}^{\ell} \boldsymbol{f}_{i}+\boldsymbol{u}_{R} .
$$

Here, $\boldsymbol{f}_{i}:=r^{\lambda_{i}} \boldsymbol{\psi}_{i}(\theta) \in H^{1}\left(\Omega, \mathbb{R}^{2}\right),(r, \theta)$ are polar coordinates with the re-entrant corner as the origin, $1 / 2 \leqslant \min \left\{\lambda_{i}\right\}_{i=1}^{\ell} \leqslant 1, \boldsymbol{\psi}_{i}(\theta)$ is $C^{\infty}$ in the appropriate range of $\theta$, and $\boldsymbol{u}_{R} \in H^{k}\left(\Omega, \mathbb{R}^{2}\right)$ for some $k>1$ corresponding to $\max \left\{\lambda_{i}\right\}_{i=1}^{\ell}$. This particular form of the solution results in a singularity

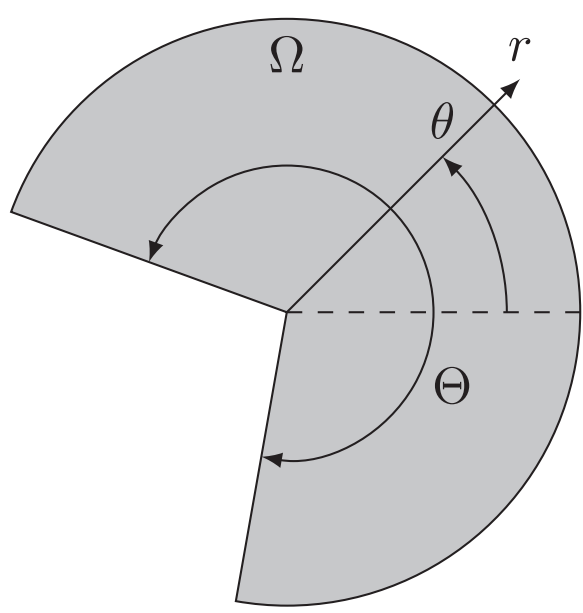

Figure 1. Domain with a re-entrant corner. 
as $u \notin H^{2}\left(\Omega, \mathbb{R}^{2}\right)$. In the particular case of fracture mechanics, $\Theta=2 \pi$ and $\lambda_{1}=1 / 2$. If $\ell=1$ is chosen, then $k=2$, a well known result obtained by Grisvard [28, 29].

The lack of regularity of the solution is reflected in poor convergence rates when standard Lagrange finite element methods are used to solve the problem. Generally, for second-order elliptic problems, the convergence rate of standard finite element methods in the $H^{1}$-seminorm is bounded by $h^{\min \{p, s-1\}}$, where $h$ is the maximum size of the elements, $p$ denotes the order of complete polynomials in the basis functions, and $s$ is such that the elastic solution $\boldsymbol{u} \in H^{s}\left(\Omega, \mathbb{R}^{2}\right)$. As a result, a necessary condition for optimal convergence is $p \leqslant s-1$. For example, for $p=1$, the minimum possible choice, $s$ must be at least 2 for optimal convergence. However, for the problem at hand, it can be shown that $\boldsymbol{u} \in H^{3 / 2-\delta}\left(\Omega, \mathbb{R}^{2}\right)$ for any $\delta>0$ but $\boldsymbol{u} \notin H^{3 / 2}\left(\Omega, \mathbb{R}^{2}\right)$. Thus, the error in the solution of the displacement gradient is of order $\mathcal{O}\left(h^{1 / 2}\right)$ (e.g., Figure 7(a)), independent of $p$, the order of the polynomial interpolant. That is to say, no matter how high the degree of the polynomial basis functions is, the convergence rate is the same and is always suboptimal.

\subsection{Our solution}

Given the known form of singularity of the solution (1), and considering that finite element solutions converge rather rapidly to the exact one if the latter is sufficiently regular, the underlying idea of the method is to construct a smooth solution by appropriately scaling the singular one. Namely, we would like to construct a bijective map $\gamma: \mathbb{R}^{2} \rightarrow \mathbb{R}^{2}$ such that $\gamma: \hat{\Omega} \rightarrow \Omega$ and that $\hat{\boldsymbol{u}}:=\boldsymbol{u} \circ \gamma \in H^{k}\left(\hat{\Omega}, \mathbb{R}^{2}\right)$, for some $k \geqslant 2$. Moreover, it will be shown later that $\hat{\boldsymbol{u}}$ satisfies a differential equation of the same type as that satisfied by $\boldsymbol{u}$, with different coefficients. Once we have constructed such a map, we

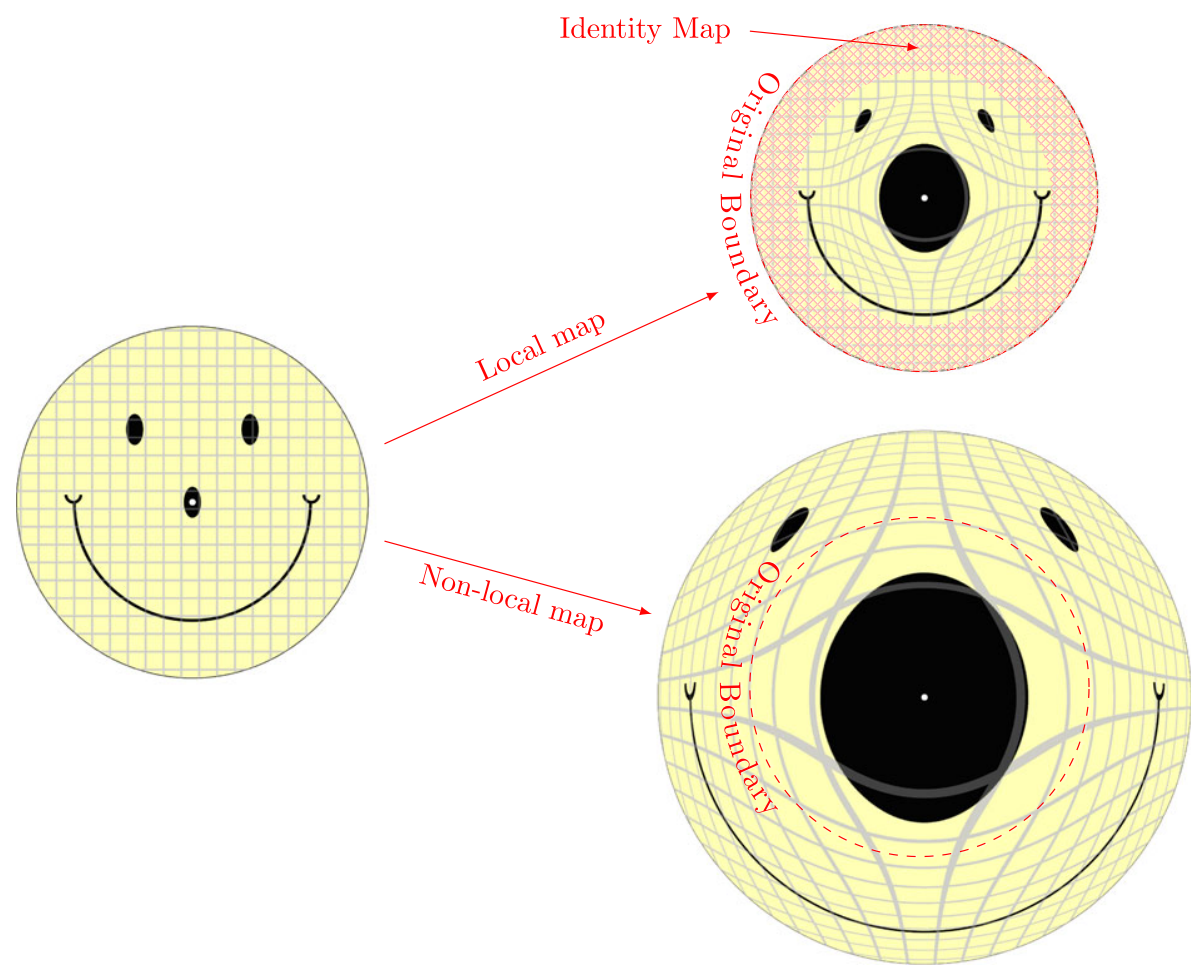

Figure 2. To 'remove' a singularity in the elasticity solution, assumed to be placed at the 'nose' in the figure, we construct a map from the original domain (left) to a parametric one (either one on the right). A general map would deform the boundary of the domain (bottom right figure) as well as deform the neighborhood of other possible singularities, such as when in the presence of multiple crack tips. Instead, the map we adopt is localized around each singularity (top right figure), which means that it equals the identity beyond a certain distance from the singularity (indicated by the cross-hatched area). This property simplifies the enforcement of boundary conditions in many situations and allows for multiple crack tips to be handled simultaneously (cf. Figure 3(b) and Section 3.1.3). 
can solve for $\hat{\boldsymbol{u}}$ using standard finite element methods, recovering optimal convergence rates of the error in $\hat{\boldsymbol{u}}$ over the parametric domain $\hat{\Omega}$. It will be later shown in Section 5 that the optimality of convergence also holds on $\Omega$ for the error in $\boldsymbol{u}$.

We are thus faced with the challenge of constructing a map $\gamma$ such that the composed solution $\hat{\boldsymbol{u}}=\boldsymbol{u} \circ \boldsymbol{\gamma}$ is sufficiently regular. The minimum regularity requirement will be dictated by the order of the interpolating polynomial.

To demonstrate the idea, consider a problem with one re-entrant corner; see Figure 1, with a crack tip as a special case. Let $(r, \theta)$ and $(\hat{r}, \hat{\theta})$ be polar coordinates associated with the re-entrant corner over $\Omega$ and $\hat{\Omega}$, respectively (e.g., Figure 3 ). The map constructed as

(a)
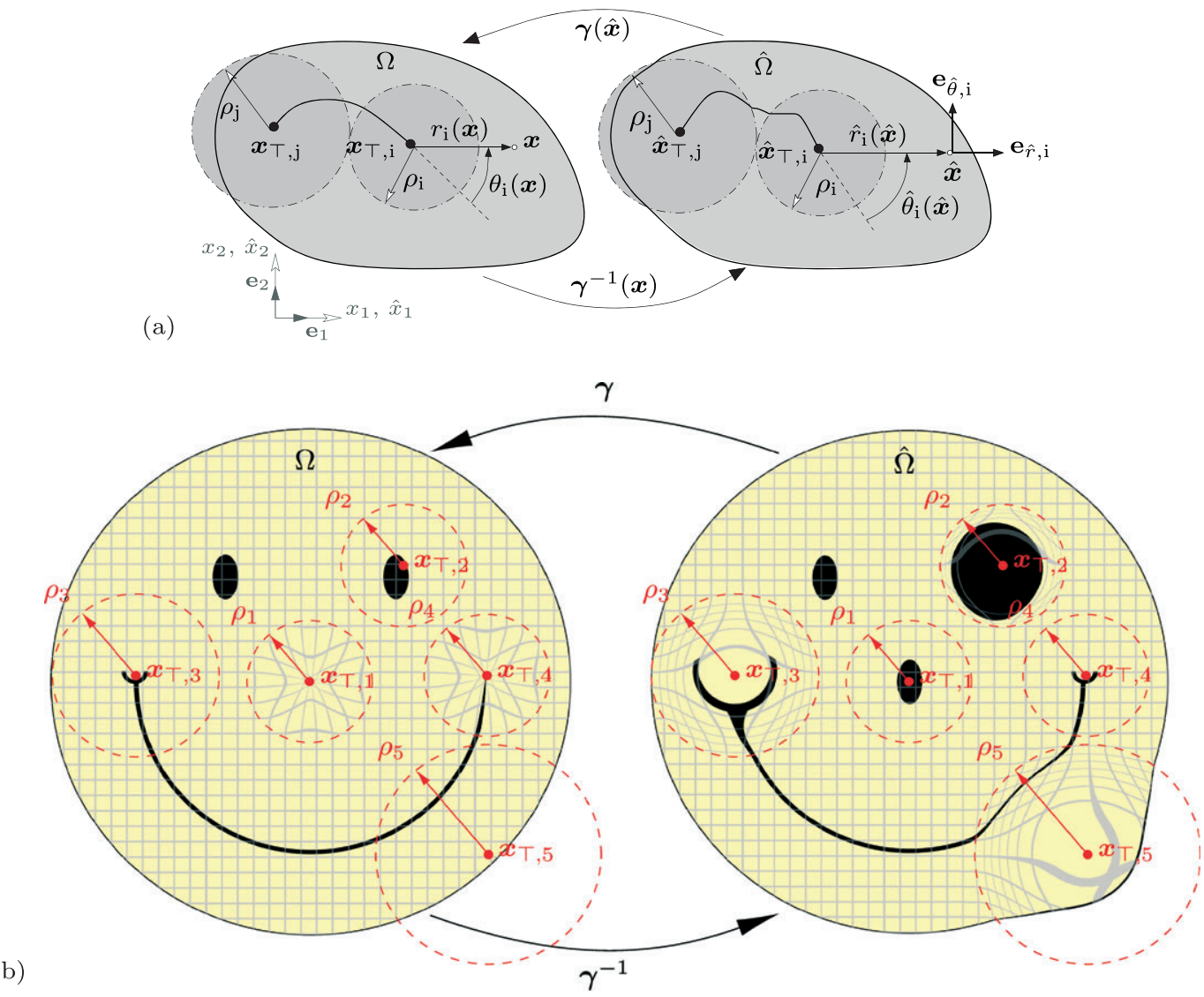

Figure 3. Physical and parametric domains, and action of the mapping and its inverse on them. While in the text, the location of the crack tips coincide;for clarity in the figure, the two domains are depicted translated with respect to each other. (a) The problem is recast from a domain $\Omega$ to a domain $\hat{\Omega}$. The two domains coincide outside balls of radius $\rho_{\mathrm{j}}$ centered at the position of the $j$ th crack tip, $\boldsymbol{x}_{\mathrm{T}, \mathrm{j}}$. (b) Illustration of the action of the mapping $\gamma$. The image on the left shows the physical domain $\Omega$, while the right figure shows the parametric domain $\hat{\Omega}$, namely, the image of the physical domain under the action of the inverse mapping $(\hat{\Omega}=$ $\gamma^{-1}(\Omega)$ ). We label with $x_{\mathrm{T}, \mathrm{i}}, i=1 \ldots 5$ the singularities around which we like to perform a parameterization. Several aspects of the mapping are worthwhile highlighting: (1) the mapping, as detailed in Section 3.1.3, is such that the parameterization is localized to the ball of radius $\rho_{\mathrm{i}}$ centered at $\boldsymbol{x}_{\mathrm{T}, \mathrm{i}}, B_{\rho_{\mathrm{i}}}\left(\boldsymbol{x}_{\mathrm{T}, \mathrm{i}}\right)$, such that $B_{\rho_{\mathrm{i}}}\left(\boldsymbol{x}_{\mathrm{T}, \mathrm{i}}\right) \cap$ $B_{\rho_{\mathrm{j}}}\left(\boldsymbol{x}_{\mathrm{T}, \mathrm{j}}\right)=\varnothing$ for all $i \neq j$, allowing a simple way to handle multiple singularities; (2) the boundary of the parametric domain $(\partial \hat{\Omega})$ and that of the physical domain $(\partial \Omega)$ do not necessarily coincide, as boundary conditions can be easily handled through map composition and proper integral scaling (cf. (12a) and (14b)); (3) moving from the domain $\Omega$ to $\hat{\Omega}$, points close to the singularity are 'pulled away' (effectively allowing the singular function to possess lower gradients when mapped to $\hat{\Omega}$ ); and (4) moving from the domain $\hat{\Omega}$ to the domain $\Omega$ the points farther from the singularity are brought closer to the singularity (effectively allowing for a smooth function defined over $\hat{\Omega}$ to possess sharp features over $\Omega$ ). This last observation is responsible for the 'disappearance' of the nose and the right dimple in $\hat{\Omega}$ when mapped to $\Omega$. 


$$
\gamma:(\hat{r}, \hat{\theta}) \mapsto\left(r=\hat{r}^{4}, \theta=\hat{\theta}\right)
$$

transforms the function $r^{\lambda_{1}}$ over $\Omega$ to $\hat{r}^{4 \lambda_{1}}$ over $\hat{\Omega}$. Recall that because $\lambda_{1} \geqslant 1 / 2$, it follows that $\hat{r}^{4 \lambda_{1}}$ is in $H^{2}(\hat{\Omega})$ (Section 5). As a result, if $P^{1}$ shape functions are used to solve for $\hat{\boldsymbol{u}}$, then optimal convergence is expected. In the actual method, we will localize the map via a cutoff function so that multiple singularities can be independently handled. Moreover, we will build the map according to the order of interpolating polynomials to ensure optimal convergence. Figure 2 showcases the effects of localizing the map around a specific point, in contrast to reparameterizing the entire domain. More details will follow in Section 3.1.3, as well as in Figure 3.

In the following section, we provide a detailed description of the construction of the smooth problem, as well as its numerical solution via standard Lagrange finite element methods. It will become apparent to the reader that the method can be easily implemented with minor alterations to any standard finite element program, effectively yielding optimal convergence rates with minimal computational and implementation overburden.

\section{THE METHOD}

With the overview of the method in mind from Section 2, we proceed next to present a detailed construction of the problem over the parametric domain and its numerical solution.

We begin by stating in Section 3.1.1 the elasticity problem over the domain occupied by the body of interest, followed by, in Sectin 3.1.2, an equivalent weak formulation over a generic parametric domain $\hat{\Omega}$ (with some mild restriction). We then discuss in Section 3.1.3 the construction of the map $\gamma: \hat{\Omega} \rightarrow \Omega$ that ensures a sufficiently smooth solution of the problem over $\hat{\Omega}$. We lastly conclude the section with the introduction of the discrete problem statement in Section 3.2 and a summary of the method in Section 3.3.

\subsection{Constructing the problem in the parametric domain}

3.1.1. Problem statement in the physical domain. We consider a linear elasticity problem over a bounded open domain $\Omega \subset \mathbb{R}^{2}$ with an associated Cartesian coordinate system $x_{i}, i=1,2$. The problem reads: Find $\boldsymbol{u}: \Omega \rightarrow \mathbb{R}^{2}$ such that

$$
\begin{aligned}
\nabla \cdot(\mathbb{C}: \nabla \boldsymbol{u})+\boldsymbol{b} & =0, & & \text { in } \Omega, \\
\boldsymbol{u} & =\boldsymbol{g}, & & \text { on } \partial_{d} \Omega, \\
(\mathbb{C}: \nabla \boldsymbol{u}) \cdot \boldsymbol{n} & =\boldsymbol{t}, & & \text { on } \partial_{\tau} \Omega,
\end{aligned}
$$

where $\boldsymbol{n}$ denotes the unit outward normal to the boundary $\partial \Omega=\overline{\partial_{d} \Omega \cup \partial_{\tau} \Omega}, \partial_{d} \Omega \cap \partial_{\tau} \Omega=\emptyset, \partial_{d} \Omega$ has a positive length, $g: \partial_{d} \Omega \rightarrow \mathbb{R}^{2}$ denotes the prescribed boundary displacement, $\boldsymbol{t}: \partial_{\tau} \Omega \rightarrow \mathbb{R}^{2}$ denotes the prescribed boundary tractions and $\boldsymbol{b}: \Omega \rightarrow \mathbb{R}^{2}$ represents the body force field. For the case with cracks, the domain $\Omega$ is intended as the cracked domain (namely, the crack is considered a portion of the domain boundary), and we let $\mathscr{C}=\partial \Omega \backslash \partial \bar{\Omega}$ denote the crack and ensure that $\partial_{\tau} \Omega \supseteq \mathscr{C}$. The constitutive tensor $\mathbb{C}$ is defined as

$$
\mathbb{C}=\Lambda \mathbf{1} \otimes \mathbf{1}+2 \mu \mathbb{I}, \quad \Lambda= \begin{cases}\lambda, & \text { for plane strain, } \\ \frac{2 \lambda \mu}{\lambda+2 \mu}, & \text { for plane stress, }\end{cases}
$$

where $\lambda$ and $\mu$ are Lamé's first and second parameters, which we take as strictly positive, and $\mathbf{1}$ and $\mathbb{I}$ are the second order identity tensor and the fourth-order symmetric identity tensor, respectively, as defined in Section 1.

For the set of admissible displacements $\mathcal{V}$ and that of test functions $\mathcal{W}$ defined as

$$
\begin{gathered}
\mathcal{V}=\left\{\boldsymbol{u} \in H^{1}\left(\Omega, \mathbb{R}^{2}\right) \mid \boldsymbol{u}=\boldsymbol{g} \text { on } \partial_{d} \Omega\right\}, \\
\mathcal{W}=\left\{\boldsymbol{w} \in H^{1}\left(\Omega, \mathbb{R}^{2}\right) \mid \boldsymbol{w}=\mathbf{0} \text { on } \partial_{d} \Omega\right\},
\end{gathered}
$$


the weak form of (2) reads: Find $\boldsymbol{u} \in \mathcal{V}$ such that

$$
a(\boldsymbol{u}, \boldsymbol{w})=F(\boldsymbol{w}), \quad \forall \boldsymbol{w} \in \mathcal{W},
$$

where

$$
\begin{aligned}
a(\boldsymbol{u}, \boldsymbol{w}) & :=\int_{\Omega} \nabla \boldsymbol{u}: \mathbb{C}: \nabla \boldsymbol{w} d \Omega, \\
F(\boldsymbol{w}) & :=\int_{\Omega} \boldsymbol{b} \cdot \boldsymbol{w} d \Omega+\int_{\partial_{\tau} \Omega} \boldsymbol{t} \cdot \boldsymbol{w} d \Gamma .
\end{aligned}
$$

3.1.2. Problem statement in the parametric domain. Let $\gamma: \mathbb{R}^{2} \rightarrow \mathbb{R}^{2}$ be a diffeomorphism such that $\Omega=\gamma(\hat{\Omega})$ and that $\gamma=$ id outside a sufficiently large ball containing all crack tips and re-entrant corners, where id denotes the identity map. Additionally, we introduce for later use a set of Cartesian coordinates $\hat{x}_{i}$ associated with $\hat{\Omega}$ (cf. Figure 3(a)). With

$$
\hat{\mathcal{V}}:=\mathcal{V} \circ \gamma, \quad \hat{\mathcal{W}}:=\mathcal{W} \circ \gamma
$$

we have an equivalent formulation of (3) given by the following: Find $\hat{\boldsymbol{u}} \in \hat{\mathcal{V}}$ such that

$$
a_{\gamma}(\hat{\boldsymbol{u}}, \hat{\boldsymbol{w}})=\hat{F}(\hat{\boldsymbol{w}}), \quad \forall \hat{\boldsymbol{w}} \in \hat{\mathcal{W}},
$$

where

$$
\begin{aligned}
& a_{\gamma}(\hat{\boldsymbol{u}}, \hat{\boldsymbol{w}}):=\int_{\hat{\Omega}} \nabla \hat{\boldsymbol{u}}: \mathbb{M}: \nabla \hat{\boldsymbol{w}} d \hat{\boldsymbol{\Omega}},
\end{aligned}
$$

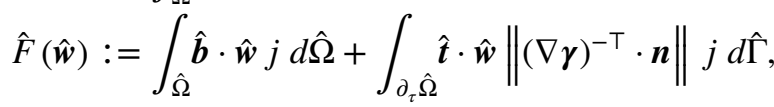

and $j:=\operatorname{det}(\nabla \gamma)$ denotes the Jacobian of the mapping $\gamma$.

The symbol^ ${ }^{\wedge}$ is here reserved for functions composed with $\boldsymbol{\gamma}$, for example, $\hat{\boldsymbol{u}}=\boldsymbol{u} \circ \boldsymbol{\gamma}$, and

$$
\mathbb{M}:=j(\nabla \gamma)^{-1} \cdot \mathbb{C} \cdot(\nabla \gamma)^{-\top} .
$$

We remark that $\mathbb{M}$ possesses the major symmetry but not necessarily the minor symmetries as it can be readily seen from its expanded form

$$
\mathbb{M}=\Lambda j(\nabla \gamma)^{-1} \otimes(\nabla \gamma)^{-\top}+2 \mu j(\nabla \gamma)^{-1} \cdot \mathbb{I} \cdot(\nabla \gamma)^{-\top} .
$$

The operator $a_{\gamma}$ was constructed so that

$$
a(\boldsymbol{u}, \boldsymbol{w})=a_{\gamma}(\hat{\boldsymbol{u}}, \hat{\boldsymbol{w}}),
$$

for $\hat{\boldsymbol{u}}=\boldsymbol{u} \circ \boldsymbol{\gamma}$ and $\hat{\boldsymbol{w}}=\boldsymbol{w} \circ \gamma$.

3.1.3. The map. The regularity of the solution of the problem stated in (4) is predicated on the appropriate construction of the mapping $\gamma$. In this section, we will discuss the construction of such mapping. For clarity, we first present the construction of $\gamma$ for the case of a single singularity (crack tip or re-entrant corner); then, we generalize it to handle multiple singularities.

3.1.3.1. The case of a single singularity. Let $\boldsymbol{x}_{\mathrm{T}}$ be the location of the singularity in the physical domain, and by construction, also the location of the singularity in the parametric domain, that is, $\boldsymbol{\gamma}\left(\boldsymbol{x}_{\top}\right)=\boldsymbol{x}_{\mathrm{\top}}$. We define $\hat{\boldsymbol{r}}=\hat{\boldsymbol{r}}(\hat{\boldsymbol{x}}):=\left\|\hat{\boldsymbol{x}}-\boldsymbol{x}_{\top}\right\|$, and construct the mapping as

$$
\gamma(\hat{\boldsymbol{x}})=\boldsymbol{x}_{\mathrm{\top}}+\frac{q(\eta)}{\eta}\left(\hat{\boldsymbol{x}}-\boldsymbol{x}_{\mathrm{T}}\right), \quad \eta=\eta(\hat{\boldsymbol{x}}):=\frac{\hat{r}(\hat{\boldsymbol{x}})}{\rho},
$$

for some $\rho \in \mathbb{R}^{+}$and

$$
q(\eta)= \begin{cases}\eta\left[1-\exp \left(1+\frac{1}{\eta^{2 m-1}-1}\right)\right], & \text { if } \eta<1, \\ \eta, & \text { otherwise }\end{cases}
$$


with $m \geqslant 2$. The value of $m$ is chosen so that $m \geqslant p+1$, where $p$ is the order of polynomials in the finite element space (see Section 3.2 for specifics). In this way, optimal convergence of the finite element approximation follows (see Section 5). For example, if piecewise quadratic polynomials to construct approximations over $\hat{\Omega}$ are selected, $p=2$, then we would set $m=3$.

To connect (6) with the discussion in Section 2.2, we first recall $r=r(\boldsymbol{x}):=\left\|\boldsymbol{x}-\boldsymbol{x}_{\mathrm{T}}\right\|$. From (6), we can conclude that

$$
\frac{r}{\rho}=\frac{1}{\rho}\left\|\gamma(\hat{\boldsymbol{x}})-\gamma\left(\boldsymbol{x}_{\mathrm{\top}}\right)\right\|=\frac{1}{\rho}\left\|\hat{\boldsymbol{x}}-\boldsymbol{x}_{\mathrm{\top}}\right\| \frac{q(\hat{r} / \rho)}{\hat{r} / \rho}=\frac{\hat{r}}{\rho} \frac{q(\hat{r} / \rho)}{\hat{r} / \rho}=q\left(\frac{\hat{r}}{\rho}\right),
$$

and hence that $q(\hat{r} / \rho)$ plays the role of 'stretching' $\hat{r}$ into $r$. As we shall see, the choice of $q(\eta)$ in (7) guarantees that

(i) We have $r \sim \hat{r}^{2 m}$ as $\hat{r} \searrow 0$, which is the condition stated in Section 2.2 to have $\hat{\boldsymbol{u}} \in H^{k}\left(\hat{\Omega}, \mathbb{R}^{2}\right)$, with $k \geqslant 2$.

(ii) The mapping $\gamma$ is bijective.

(iii) The mapping $\gamma$ is the identity outside a compact region containing the singularity.

By defining $\gamma$, we defined $\hat{\Omega}=\gamma^{-1}(\Omega)$ as well. As a result, if the singularity is located at the tip of a curved crack, the path of the crack will be different in $\hat{\Omega}$ than in $\Omega$. We illustrate this with examples later.

Remark 1 (Generalization)

The choice of $q(\eta)$ in (7) is not the only one. More generally, we can choose any function $q(\eta) \in \Pi_{k, m}$, with $m \geqslant k \geqslant 2$ and

$$
\begin{aligned}
\Pi_{k, m}:=\{ & \left\{\in C^{k}\left(\mathbb{R}_{0}^{+}\right) \mid q(\eta)=\eta \forall \eta>1 ;\right. \\
& q^{(j)}(0)=0, j=0, \ldots, k-1 ; \quad q^{\prime}(\eta) \geqslant 0 \forall \eta \geqslant 0 ; \\
& \left.\exists C_{1}, C_{2}, \eta_{0}>0 \quad \text { s.t. } q^{\prime}(\eta) \geqslant C_{1} \eta^{2 m-1} \text { and }\left|q^{(k)}(\eta)\right| \leqslant C_{2} \eta^{2 m-k} \forall \eta \in\left[0, \eta_{0}\right]\right\} .
\end{aligned}
$$

It is simple to build $q \in \Pi_{k, m}$ with polynomials, for example. Here, $k$ is the desired degree of smoothness of the mapped solution near the singularity, that is, $\hat{\boldsymbol{u}} \in H^{k}(\hat{\Omega})$. For the numerical approximation of the solution, the smoother the function, the higher the attainable order of convergence. Hence, in general, we will select $m=k=p+1$, where $p$ is the order of the polynomials in the finite element space.

The conditions stated in (9) imply that $0 \leqslant q(\eta) / \eta \leqslant 1$ for all $\eta \in \mathbb{R}_{0}^{+}$. Moreover, by integration, it is simple to see that they also constrain the behavior of $q$ near $\eta=0$. More precisely, there exist $C_{3}, C_{4}>0$ and $\eta_{0}>0$ such that $\forall \eta \in\left[0, \eta_{0}\right]$,

$$
\begin{gathered}
C_{3} \eta^{2 m} \leqslant q(\eta) \leqslant C_{4} \eta^{2 m}, \\
C_{3} \eta^{2 m-1} \leqslant q^{\prime}(\eta), \\
\left|q^{(j)}(\eta)\right| \leqslant C_{4} \eta^{2 m-j}, \quad j=1, \ldots, k .
\end{gathered}
$$

Together, (10a) and (8) show precisely that $r \sim \hat{r}^{2 m}$ near 0 , while (10c) guarantees that the derivatives of $\gamma$ do not grow more than the derivatives of $\hat{r}^{2 m}$ do. Equation (10b) ensures the growth of the gradient of $\gamma^{-1}$, which participates in $\mathbb{M}$; see Section 5, as well as the bijectivity of $\gamma$. The bijectivity of $\gamma$ is readily seen from (8). In fact, for $\hat{r} \in[0, \rho]$,

$$
\frac{d r}{d \hat{r}}=q^{\prime}\left(\frac{\hat{r}}{\rho}\right) \geqslant 0
$$

with the equal sign satisfied if and only if $\eta=0$, implying the bijectivity of $\gamma$. 
The discussion in Section 2.2 corresponds to a very simple $q$ that takes the form $q(\eta)=\eta^{4}$ for all $\eta \geqslant 0$. This $q$ satisfies all conditions in (9) with $k=m=2$, except $q(\eta)=\eta$ for $\eta>1$, and leads to simpler computations of the gradient of the map. However, it is convenient to choose a $q$ that coincides with the identity for $\eta$ large enough because it permits localizing the map to each singularity, an appealing feature when dealing with multiple singularities.

For the remainder of the manuscript, we use (7) as the function $q$ of choice. We do so because (7) is simple to evaluate, and it can be used to construct approximations of any order, given that it is $C^{\infty}$. The parameter $\rho$ should be chosen to be at least a few times the radius of curvature of $\partial \Omega$ at $\boldsymbol{x}_{\mathrm{T}}$, so that the mesh size required to resolve the near-tip behavior is not smaller than that needed to resolve the geometry.

3.1.3.2. Handling multiple singularities. Let $t \in \mathbb{N}$ denote the number of singularities, located at $\boldsymbol{x}_{\mathrm{T}, \tau} \in \bar{\Omega}, \tau=1, \ldots, t$. For each $\tau$, we set $r_{\tau}: \boldsymbol{x} \mapsto\left\|\boldsymbol{x}-\boldsymbol{x}_{\mathrm{T}, \tau}\right\|$; see Figure 3. In this particular case, we assume that the solution can be written as the sum of the asymptotic expansions about each singularity plus the regular part as

$$
\boldsymbol{u}=\sum_{\tau=1}^{t} \sum_{j=1}^{\ell_{\tau}} r_{\tau}^{\lambda_{j}} \boldsymbol{\psi}_{j, \tau}\left(\theta_{\tau}\right)+\boldsymbol{u}_{R},
$$

where $\theta_{\tau}$ is a polar angle associated with $\boldsymbol{x}_{\mathrm{\top}, \tau}$ and, as before, $\boldsymbol{\psi}_{j, \tau}$ and $\boldsymbol{u}_{R}$ are assumed to be sufficiently smooth.

To account for each singularity, we then construct the mapping $\gamma: \mathbb{R}^{2} \rightarrow \mathbb{R}^{2}$ as

$$
\boldsymbol{\gamma}(\hat{\boldsymbol{x}})=\hat{\boldsymbol{x}}-\sum_{\tau=1}^{t}\left(1-\frac{q\left(\eta_{\tau}\right)}{\eta_{\tau}}\right)\left(\hat{\boldsymbol{x}}-\boldsymbol{x}_{\mathrm{T}, \tau}\right),
$$

where $\eta_{\tau}=\eta_{\tau}(\hat{\boldsymbol{x}}):=\hat{r}_{\tau}(\hat{\boldsymbol{x}}) / \rho_{\tau}$, with $\hat{r}_{\tau}: \hat{\boldsymbol{x}} \mapsto\left\|\hat{\boldsymbol{x}}-\boldsymbol{x}_{\mathrm{T}}\right\|$. We choose $\left\{\rho_{\tau}\right\}_{\tau} \in\left(\mathbb{R}^{+}\right)^{t}$ within the sole restriction that $B_{\rho_{\tau}}\left(\boldsymbol{x}_{\mathrm{T}, \tau}\right) \cap B_{\rho_{\tau^{\prime}}}\left(\boldsymbol{x}_{\mathrm{T}, \tau^{\prime}}\right)=\emptyset, \forall \tau \neq \tau^{\prime}$, where $B_{\rho}(\mathbf{y})=\left\{\boldsymbol{x} \in \mathbb{R}^{2} \mid\|\boldsymbol{x}-\mathbf{y}\|<\rho\right\}$ is the ball of radius $\rho$ around $\mathbf{y}$; in other words, we require that the neighborhoods of the singularities being mapped are mutually disjoint. Hence, by construction, the mapping is local to each singularity and the mapped solution possesses the desired regularity. Figure 4 illustrates the construction of such mappings for a one-dimensional analog of the problem.

The expression of the gradient of the mapping $\gamma$ is provided in Appendix A.

\subsection{The numerical approximation}

In the following, we describe the construction of a numerical approximation to $\hat{\boldsymbol{u}}$ and from that an approximation to $\boldsymbol{u}$.

Let $\hat{\Omega}^{h}$ be an approximation of $\hat{\Omega}$ of the appropriate order so that the error introduced by the approximation of the domain does not dominate the order of convergence of the method. For example, a standard isoparametric approximation of the domain would suffice. A similar consideration is assumed in constructing the approximation $\partial_{d} \hat{\Omega}^{h}$ of $\partial_{d} \hat{\Omega}=\gamma^{-1}\left(\partial_{d} \Omega\right)$, then setting $\partial_{\tau} \hat{\Omega}^{h}=\partial \hat{\Omega}^{h} \backslash \partial_{d} \hat{\Omega}^{h}$. This can be easily accomplished if, for example, $\partial_{d} \Omega=\partial \Omega$, in which case we set $\partial_{d} \hat{\Omega}^{h}=\partial \hat{\Omega}^{h}$, or otherwise if the end points of $\partial_{d} \hat{\Omega}^{h}$ coincide with the end points of $\partial_{d} \hat{\Omega}$, and they coincide with nodes in the mesh.

Let $\mathcal{T}^{h}$ denote a finite element mesh over $\hat{\Omega}^{h}$ (Figure 5) with standard conditions, namely, (1) $K \subset \hat{\Omega}^{h}$ for all elements $K \in \mathcal{T}^{h} ;(2) \overline{\hat{\Omega}^{h}}=\overline{\cup_{K \in \mathcal{T} h} K}$, where $K$ denotes both a typical element and the open set occupied by this element; and (3) for any $K_{1}, K_{2} \in \mathcal{T}^{h}, \overline{K_{1}} \cap \overline{K_{2}}$ can only be empty, a common vertex, or a complete common edge. Here, $h=\max _{K \in \mathcal{T} h} \operatorname{diam} K$, where $\operatorname{diam} K$ denotes the diameter of $K$.

Over $\mathcal{T}^{h}$, we build the finite element spaces

$$
\begin{gathered}
\hat{\mathcal{V}}^{h, p}=\left\{\hat{\boldsymbol{u}}^{h} \in H^{1}\left(\hat{\boldsymbol{\Omega}}^{h}, \mathbb{R}^{2}\right)\left|\hat{\boldsymbol{u}}^{h}\right|_{K} \in P^{p}\left(K, \mathbb{R}^{2}\right), \hat{\boldsymbol{u}}^{h}=\hat{\mathcal{I}}^{h}(\boldsymbol{g} \circ \gamma) \text { on } \partial_{d} \hat{\boldsymbol{\Omega}}^{h}\right\}, \\
\hat{\mathcal{W}}^{h, p}=\left\{\hat{\boldsymbol{w}}^{h} \in H^{1}\left(\hat{\boldsymbol{\Omega}}^{h}, \mathbb{R}^{2}\right)\left|\hat{\boldsymbol{w}}^{h}\right|_{K} \in P^{p}\left(K, \mathbb{R}^{2}\right), \hat{\boldsymbol{w}}^{h}=\mathbf{0} \text { on } \partial_{d} \hat{\boldsymbol{\Omega}}^{h}\right\} .
\end{gathered}
$$




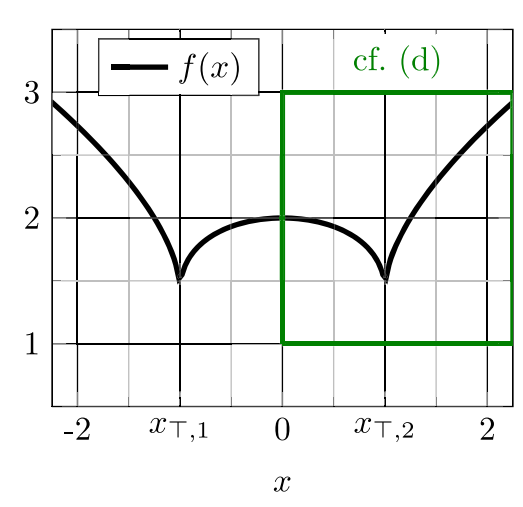

(a) The singular function.

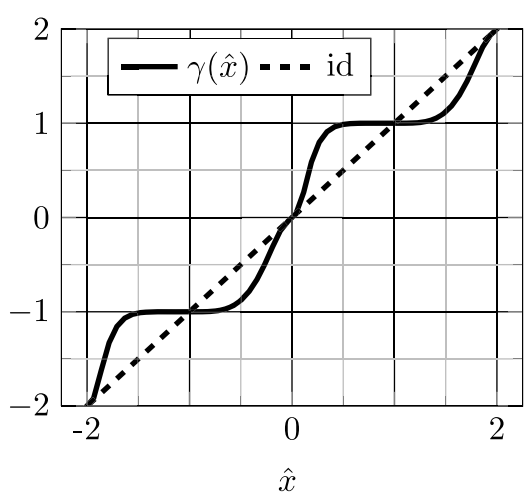

(c) The global map (c.f. Eq (11))

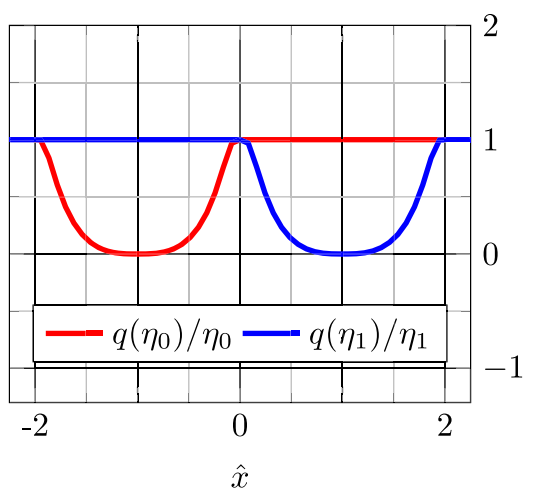

(b) Functions $q\left(\eta_{\tau}\right) / \eta_{\tau}$ with $m=2$ in Eq. (7)

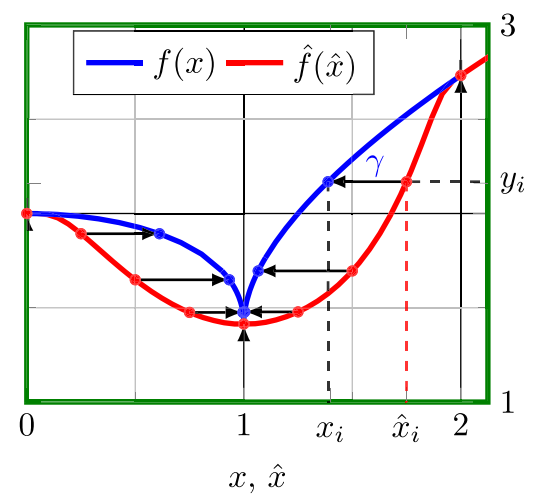

(d) The resulting mapped function over a subdomain as highlighted in (a). The markers show corresponding values of $x_{i}$ and $\hat{x}_{i}$ such that $f\left(x_{i}\right)=\hat{f}\left(\hat{x}_{i}\right)=y_{i}$.

Figure 4. One-dimensional analog of the mapping. We begin by considering in (a) the function $f(x)=\mid x-$ $\left.x_{\mathrm{T}, 1}\right|^{1 / 2}+\left|x-x_{\mathrm{T}, 2}\right|^{1 / 2}$, where $x_{\mathrm{T}, \tau}=(-1)^{\tau}, \tau=1,2$. We set $m=k=2, \rho_{\tau}=1$ and then construct in (b) the maps $q\left(\eta_{\tau}\right)$ with $\eta_{\tau}=\left|x-x_{\mathrm{T}, \tau}\right| / \rho_{\tau}$, for $\tau=1,2$. In (c), we show the global map similar to (11); and lastly in (d), we show the smooth function $\hat{f}=f \circ \gamma$ alongside the action of the mapping on a set of evenly spaced points $\left\{\hat{x}_{i}\right\}_{i}$.

Here, $P^{p}(K)$ denotes the family of complete polynomials of order up to $p \in \mathbb{N}$ over $K$, and $\hat{\mathcal{I}}^{h}$ is the interpolation operator over such elements. For Lagrange finite elements, the condition $\hat{\boldsymbol{u}}^{h}=\hat{\mathcal{I}}^{h}(\boldsymbol{g} \circ \boldsymbol{\gamma})$ on $\partial_{d} \hat{\boldsymbol{\Omega}}^{h}$ in (12a) is equivalent to $\hat{\boldsymbol{u}}^{h}=\boldsymbol{g} \circ \boldsymbol{\gamma}$ on nodes on $\partial_{d} \hat{\Omega}^{h}$. It is worthwhile noting that the piecewise polynomial functions are constructed over $\hat{\Omega}^{h}$; when composed with the mapping $\gamma$ they will no longer be polynomials.

The Galerkin form of the problem statement then reads: Find $\hat{\boldsymbol{u}}^{h} \in \hat{\mathcal{V}}^{h, p}$ such that

where

$$
a_{\gamma}^{h}\left(\hat{\boldsymbol{u}}^{h}, \hat{\boldsymbol{w}}^{h}\right)=F_{\gamma}^{h}\left(\hat{\boldsymbol{w}}^{h}\right), \quad \forall \hat{\boldsymbol{w}}^{h} \in \hat{\mathcal{W}}^{h, p},
$$

$$
\begin{gathered}
a_{\gamma}^{h}\left(\hat{\boldsymbol{u}}^{h}, \hat{\boldsymbol{w}}^{h}\right):=\int_{\hat{\boldsymbol{\Omega}}^{h}} \nabla \hat{\boldsymbol{u}}^{h}: \mathbb{M}: \nabla \hat{\boldsymbol{w}}^{h} d \hat{\boldsymbol{\Omega}}^{h}, \\
F_{\gamma}^{h}\left(\hat{\boldsymbol{w}}^{h}\right):=\int_{\hat{\boldsymbol{\Omega}}^{h}} \hat{\boldsymbol{b}} \cdot \hat{\boldsymbol{w}}^{h} j d \hat{\boldsymbol{\Omega}}^{h}+\int_{\partial_{\tau} \hat{\Omega}^{h}} \hat{\boldsymbol{w}}^{h} \cdot\left(\hat{\boldsymbol{t}}\left\|(\nabla \boldsymbol{\gamma})^{-\top} \cdot \boldsymbol{n}\right\| j\right) \circ \mathfrak{p} d \hat{\Gamma},
\end{gathered}
$$

Note that in the above, quantities in the domain integrals defined over $\hat{\Omega}$ (e.g., $\hat{\boldsymbol{b}}, j, \nabla \gamma$ ) are taken as their zero extensions to $\hat{\Omega}^{h}$, and $\mathfrak{p}: \partial \hat{\Omega}^{h} \rightarrow \partial \hat{\Omega}$ denotes the closest point projection of a point lying in $\partial \hat{\Omega}^{h}$ to $\partial \hat{\Omega}$. The mention of the extensions is purely formal, because in practice and as a result of the use of quadrature rules these extensions are (essentially) never evaluated. Finally, we obtain an 


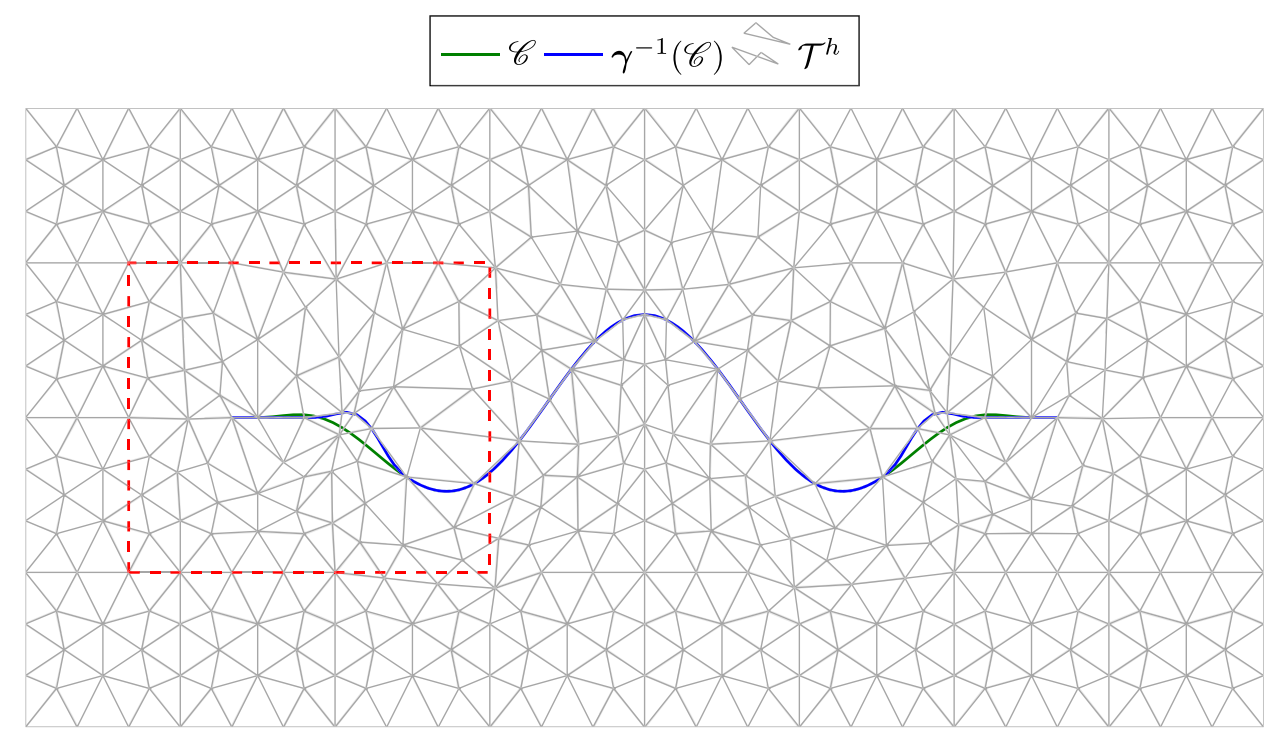

(a) Entire domain

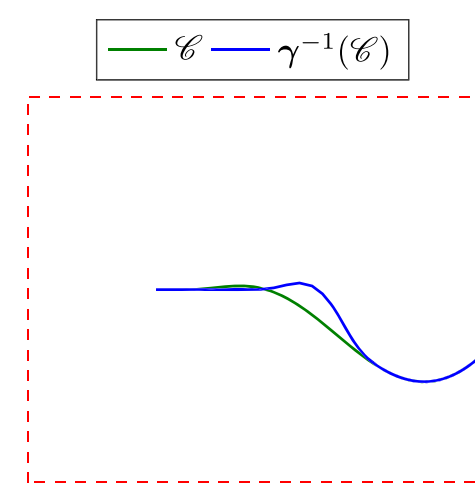

(b) Mapped boundary

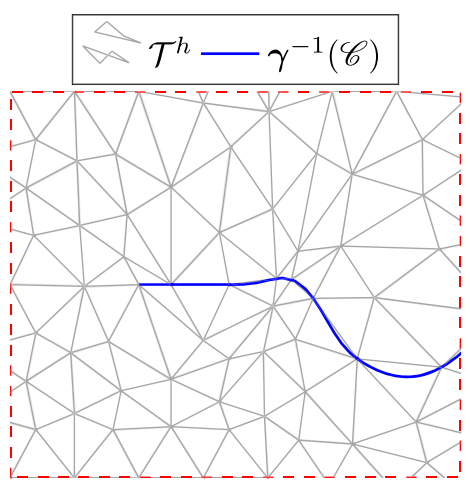

(c) Zoom in over parametric domain

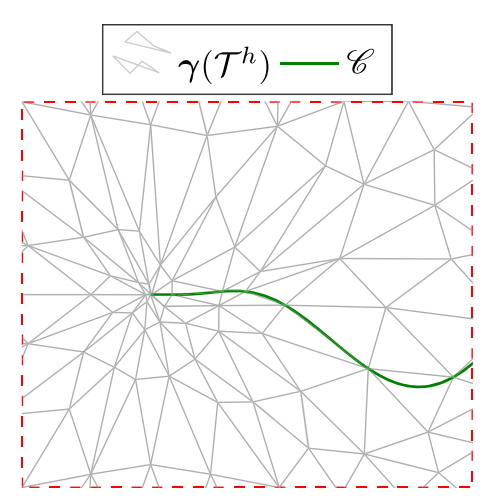

(d) Zoom in over physical domain

Figure 5. Example of a finite element mesh of $\hat{\Omega}=\gamma^{-1}(\Omega)$ with $\Omega=[-3,3] \times[-1.5,1.5] \backslash\left\{\left(x_{1}, x_{2}\right) \mid x_{1} \in\right.$ $\left.(-2,2), x_{2}=\cos \left(3 x_{1}\right) \exp \left[1 /\left(x_{1}^{2} / 4-1\right)+1\right]\right\}$. In (a), we showcase the discretization used for computations. In (b), we highlight that the boundary of the domain may not necessarily be preserved under the action of the mapping, namely, $\mathscr{C} \neq \gamma^{-1}(\mathscr{C})$. In (c), we zoom in around one of the crack tips to showcase the discretization of $\hat{\Omega}$. Lastly in (d), we showcase what the image of the discretization $\mathcal{T}^{h}$ of $\hat{\Omega}$ under the action of $\gamma$. It is worthwhile noting that $\gamma\left(\mathcal{T}^{h}\right)$ (subfigure (d)) does not serve any purpose from the computational perspective but it is solely provided to illustrate the reader the action of $\gamma$.

approximation to $\boldsymbol{u}$ as $\boldsymbol{u}^{h}=\hat{\boldsymbol{u}}^{h} \circ \gamma^{-1}$. For a more detailed description of the implementation of the method, we refer the reader to Appendix B.

\section{Remark 2 (The inverse map)}

Notice that this approximation needs a mesh over $\hat{\Omega}$ instead of over $\Omega$. To this end, an explicit expression of $\gamma^{-1}$ was derived to easily evaluate $\hat{\Omega}$ for its discretization. More precisely, the inverse map $\gamma^{-1}$ can be explicitly expressed as

for one singularity, and

$$
\boldsymbol{\gamma}^{-1}(\boldsymbol{x})=\boldsymbol{x}_{\mathrm{\top}}+\frac{q^{-1}(\hat{\eta})}{\hat{\eta}}\left(\boldsymbol{x}-\boldsymbol{x}_{\mathrm{T}}\right), \quad \hat{\eta}=\hat{\eta}(\boldsymbol{x}):=\frac{r(\boldsymbol{x})}{\rho},
$$

$$
\boldsymbol{\gamma}^{-1}(\boldsymbol{x})=\boldsymbol{x}-\sum_{\tau=1}^{t}\left(1-\frac{q^{-1}\left(\hat{\eta}_{\tau}\right)}{\hat{\eta}_{\tau}}\right)\left(\boldsymbol{x}-\boldsymbol{x}_{\mathrm{T}, \tau}\right), \quad \hat{\eta}_{\tau}=\hat{\eta}_{\tau}(\boldsymbol{x}):=\frac{r_{\tau}(\boldsymbol{x})}{\rho_{\tau}}
$$

for multiple singularities, where $q^{-1}(\hat{\eta})$ is the inverse function of $q(\eta)$. 
Remark 3 (Comparing against a method to approximate a problem with no auxiliary map) In the upcoming sections, we will recurrently contrast the approximations of $\boldsymbol{u}$ obtained with and without the auxiliary map. To be precise, we will compare the results obtained by setting $\gamma$ to (6) and (11), termed MFEM, with that obtained by setting $\gamma$ to be the identity map, which recovers a standard finite element approximation with Lagrange finite elements. For convenience, we will use the shorter acronym FEM to refer to the latter.

Notice that the MFEM does not introduce additional degrees of freedom or special shape functions over the FEM (the shape functions in the MFEM are also polynomials, but over the parametric domain).

\section{Remark 4 (Numerical computation of the bilinear form and functional)}

The bilinear form and the functional of (14a) and (14b), respectively, are performed in Section 4 using standard Gauss quadrature over a subdivision of $\hat{\Omega}^{h}$, as traditionally carried out for finite element methods.

\section{Remark 5 (Choice of $\rho \tau$ )}

In order for the results to be accurate, the radius $\rho_{\tau}$ shall be chosen to be a sufficiently large multiple of the mesh size. Of course, for convergence, once chosen, the value of $\rho_{\tau}$ should not be changed as the mesh size is refined. We observed that $\rho_{\tau} \geqslant 5 h_{0}$, where $h_{0}$ is the coarsest mesh under consideration, already yielded the asymptotic behavior where the rates of convergence approached optimal values.

\subsection{Summary of the method}

We conclude this section by providing in Box 3.1 a recapitulation of the method. We emphasize that the crucial steps are (1) the construction of the map; (2) the subdivision of the parametric domain; and (3) the generation of finite element arrays accounting for the metric changes.

\section{Box 3.1 Summary of the method.}

Step 1. For each of the $t$ singularities let $\left\{\rho_{\tau}\right\}_{\tau}$ such that $B_{\rho_{\tau}}\left(\boldsymbol{x}_{\mathrm{\top}, \tau}\right) \cap B_{\rho_{\tau^{\prime}}}\left(\boldsymbol{x}_{\mathrm{\top}, \tau^{\prime}}\right)=\emptyset, \forall \tau \neq \tau^{\prime}$.

Step 2. Select the polynomial degree $p$ of the interpolating space $\hat{\mathcal{V}}^{h, p}$.

Step 3. Choose $m \geqslant k \geqslant p+1$ (generally $m=k=p+1$ ) and generate the map $\gamma$ as

$$
\boldsymbol{\gamma}(\hat{\boldsymbol{x}})=\hat{\boldsymbol{x}}-\sum_{\tau=1}^{t}\left(1-\frac{q\left(\eta_{\tau}\right)}{\eta_{\tau}}\right)\left(\hat{\boldsymbol{x}}-\boldsymbol{x}_{\mathrm{\top}, \tau}\right),
$$

where $\eta_{\tau}:=\left\|\hat{\boldsymbol{x}}-\boldsymbol{x}_{\mathrm{T}, \tau}\right\| / \rho_{\tau}$, and

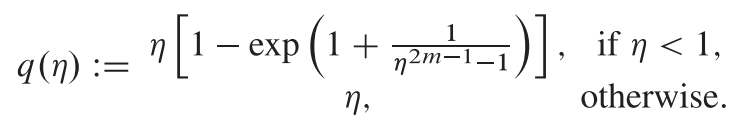

The inverse map $\gamma^{-1}$ is

$$
\boldsymbol{\gamma}^{-1}(\boldsymbol{x})=\boldsymbol{x}-\sum_{\tau=1}^{t}\left(1-\frac{q^{-1}\left(\hat{\eta}_{\tau}\right)}{\hat{\eta}_{\tau}}\right)\left(\boldsymbol{x}-\boldsymbol{x}_{\mathrm{\top}, \tau}\right),
$$

where $\hat{\eta}_{\tau}:=\left\|\boldsymbol{x}-\boldsymbol{x}_{\top, \tau}\right\| / \rho_{\tau}$.

Step 4. Generate a subdivision $\mathcal{T}^{h}$ of the approximation $\hat{\Omega}^{h}$ of $\hat{\Omega}=\gamma^{-1}(\Omega)$.

Step 5. Construct the finite element space $\hat{\mathcal{V}}^{h, p}$ over $\mathcal{T}^{h}$ with isoparametric elements.

Step 6. Solve the following problem: Find $\hat{\boldsymbol{u}}^{h} \in \hat{\mathcal{V}}^{h, p}$ such that

$$
a_{\boldsymbol{\gamma}}\left(\hat{\boldsymbol{u}}^{h}, \hat{\boldsymbol{w}}^{h}\right)=F_{\boldsymbol{\gamma}}^{h}(\hat{\boldsymbol{w}}), \quad \forall \hat{\boldsymbol{w}}^{h} \in \hat{\mathcal{W}}^{h, p} .
$$

Step 7. Compose $\hat{\boldsymbol{u}}^{h}$ with $\boldsymbol{\gamma}^{-1}$ to obtain an optimally convergent approximation to $\boldsymbol{u}$. 


\section{NUMERICAL EXAMPLES}

We next showcase the convergence of the MFEM by comparing the computed results to the analytical solutions of benchmark problems. We will further contrast the accuracy and computational complexity of the MFEM with the common FEM as introduced in Remark 3.

We considered four benchmark problems to showcase the properties of the MFEM: the first problem consists of a straight crack with loaded crack faces, the second is that of a circular arc crack, the third is that of anti-plane elastic deformations in an L-shaped domain, and lastly we consider the problem of a crack at a bi-material interface. For all problems, we show the convergence behavior of the MFEM in comparison with the FEM. We systematically provide convergence plots alongside tables with errors and computed convergence rates, for which finer meshes were obtained by successively and uniformly subdividing the coarsest mesh, which will be illustrated for each of the examples. We further provide error comparisons as functions of the problem size $N$ (namely, the number of degrees of freedom or the size of the linear system, which includes the degrees of freedom with Dirichlet boundary conditions) and CPU time taken for the assembly and the solution of the linear system, including some minor overhead tasks which take orders of magnitude less time and are independent of refinement (all the simulations were performed on a Unix based machine with $1.4 \mathrm{GHz}$ Intel Core i5 processor with $4 \mathrm{~GB} 1600 \mathrm{MHz}$ DDR3 of memory). As expected, we observe optimal convergence rates for the MFEM and sub-optimal rates for the FEM. Furthermore, we see considerable gains in accuracy for equivalent problem sizes and run times.

In what follows, we will reserve the superscript ${ }^{e}$ for the analytical solution of the boundary value problem under consideration. Furthermore, unless otherwise noted, we always let $\partial_{d} \Omega=\partial \bar{\Omega}, \boldsymbol{g}=\boldsymbol{u}^{e}$ (c.f. Equation (2)), and $\boldsymbol{b}=\mathbf{0}$. In all examples, we chose $m=k=p+1$, where $p$ is the order of the polynomials in the finite element space over the parametric configuration.

In all cases, the error $\left\|\boldsymbol{u}^{e}-\boldsymbol{u}^{h}\right\|_{0, \Omega}=\left\|\boldsymbol{u}^{e}-\hat{\boldsymbol{u}}^{h} \circ \gamma^{-1}\right\|_{0, \Omega}$ we approximated by numerical integration over $\hat{\Omega}$, namely,

$$
\left\|\boldsymbol{u}^{e}-\boldsymbol{u}^{h}\right\|_{0, \Omega} \approx\left(\left.\left.\sum\left\|\boldsymbol{u}^{e} \circ \boldsymbol{\gamma}-\hat{\boldsymbol{u}}^{h}\right\|^{2}\right|_{x_{g}} w_{g} j\right|_{x_{g}}\right)^{1 / 2}
$$

Additionally, throughout the following examples, we present further numerical results, beyond the convergence of the $L^{2}(\Omega)$-norm of the solution, that highlight additional features of the method. Namely, in Section 4.1, we discuss the conditioning of the resulting system of equation; in Section 4.2 , we discuss the convergence of the stress intensity factors; and lastly, in Section 4.4, we showcase the optimality of convergence of the derivatives of the solution measured as the $H^{1}(\Omega)$-norm $\left(\|\bullet\|_{1, \Omega}\right)$.
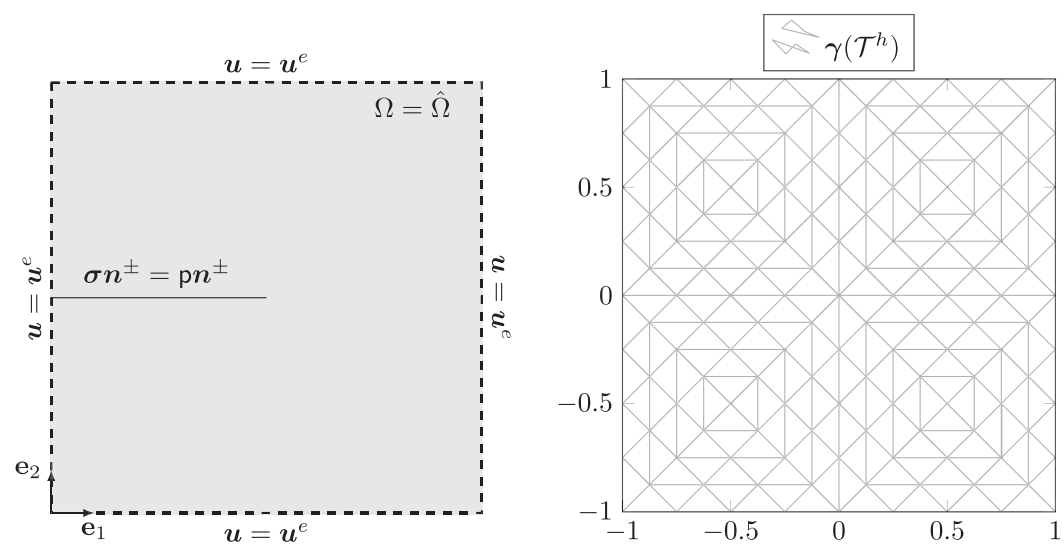

(a) Modeled subdomain with boundary conditions (b) Sample mesh corresponding to the first subdivision

Figure 6. Straight crack problem. (a) Modeled subdomain with boundary conditions; (b) sample mesh corresponding to the first subdivision. 


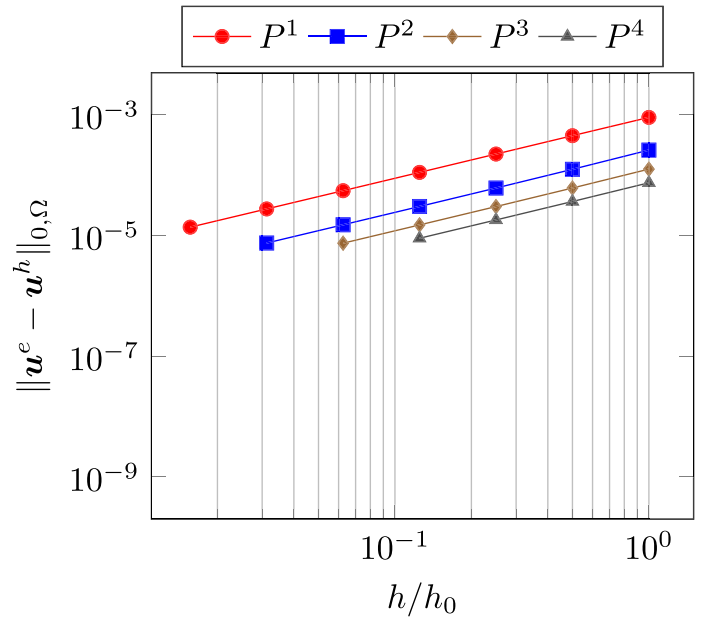

(a) Convergence in mesh size for FEM

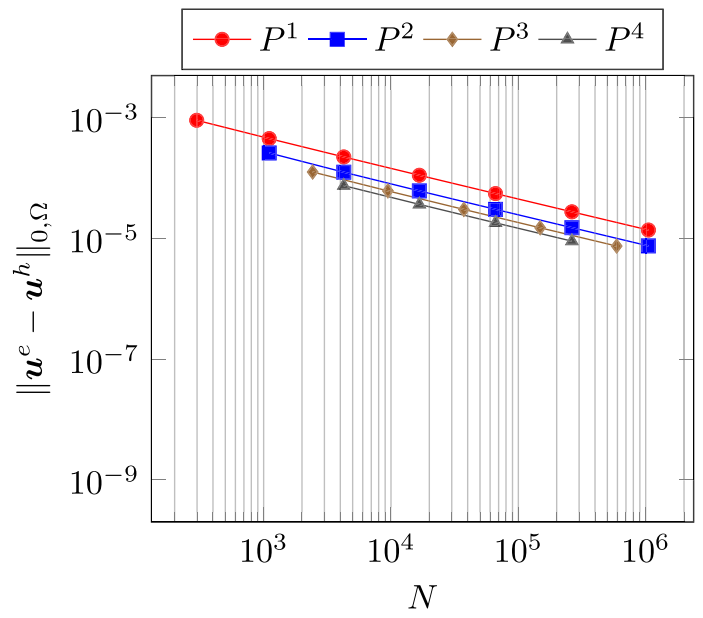

(c) Convergence in system size for FEM

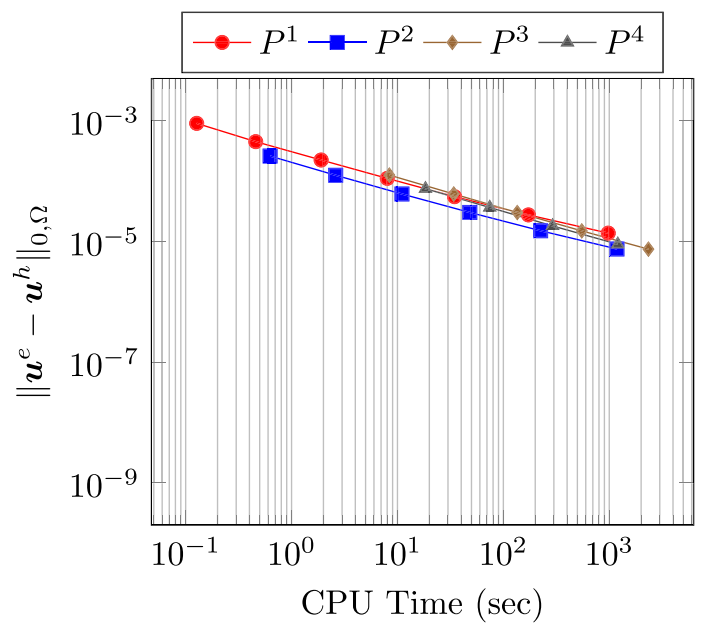

(e) Convergence in CPU time for FEM

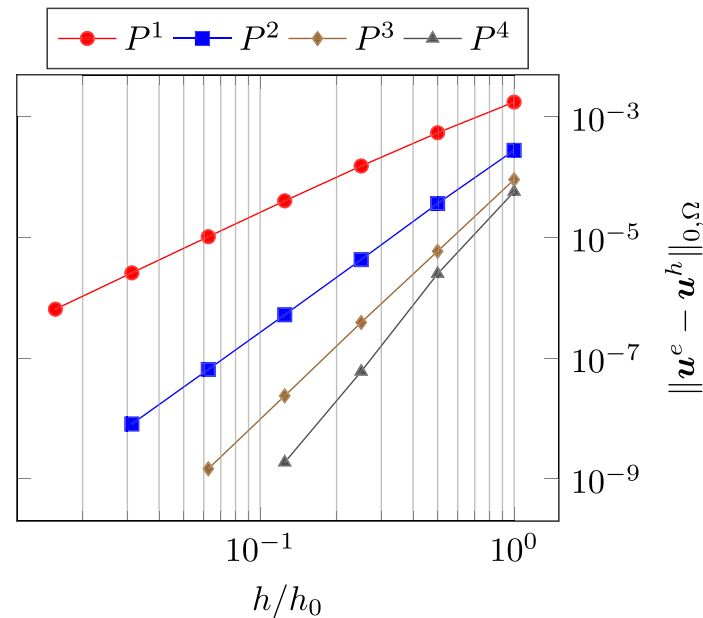

(b) Convergence in mesh size for MFEM

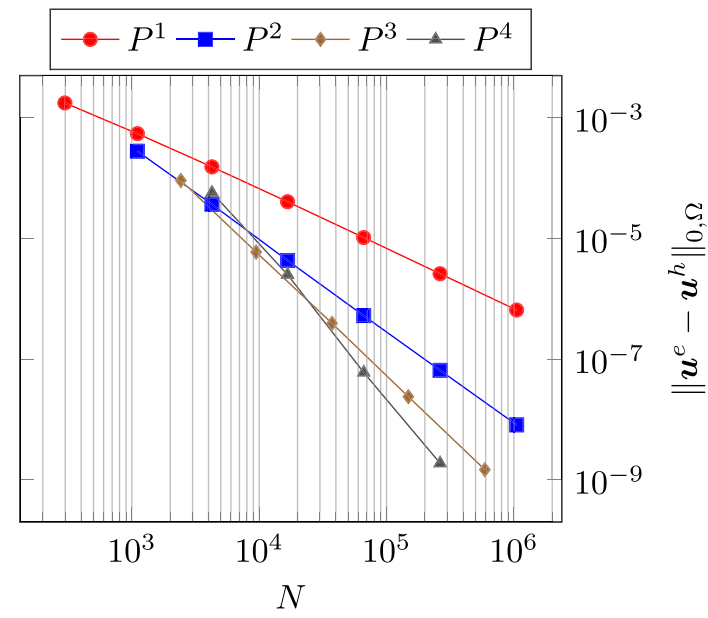

(d) Convergence in system size for MFEM

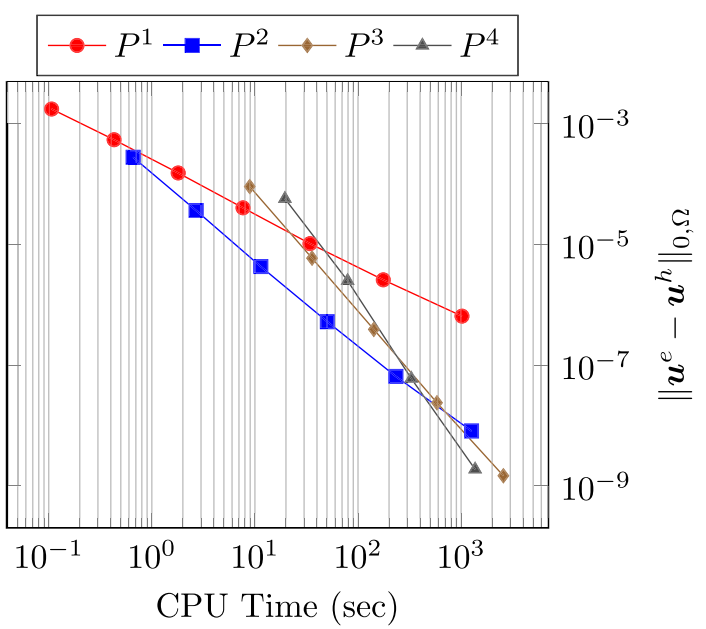

(f) Convergence in CPU time for MFEM

Figure 7. Convergence of the elasticity solution for a straight crack. (a) Convergence in mesh size for FEM; (b) convergence in mesh size for MFEM; (c) convergence in system size for FEM; (d) convergence in system size for MFEM; (e) convergence in CPU time for FEM; (f) convergence in CPU time for MFEM. 
Both triangular and quadrilateral elements are used in the presentation to showcase the independence of the optimality of convergence on the choice of the finite elements.

\subsection{Straight crack}

The first problem is the one of a semi-infinite straight crack in an infinite medium subject to mixed-mode loading. An analytical solution for the problem can be found in [30, 31]. We further superimpose a uniform pressure field to verify the application of boundary tractions in the mapped region. If we let $\boldsymbol{u}^{\mathrm{I}, \mathrm{II}}$ denote the solution field as found in [30, 31], then the solution of the example will be given by

$$
\boldsymbol{u}^{e}=\boldsymbol{u}^{\mathrm{I}, \mathrm{II}}+\boldsymbol{u}^{\mathrm{p}}
$$

where $\boldsymbol{u}^{\mathfrak{p}}$ is the displacement field associated with the uniform pressure. Assuming a plane strain state, we have

$$
\boldsymbol{u}^{\mathrm{p}}=\mathrm{p} \frac{\left(x_{1} \mathbf{e}_{1}+x_{2} \mathbf{e}_{2}\right)}{2(\Lambda+\mu)}
$$

with $p=1$ being the uniform in-plane pressure.

We model a subdomain of the infinite medium $\Omega=(-1,1)^{2} \backslash\left\{\left(x_{1}, x_{2}\right) \mid x_{2}=0, x_{1} \leqslant 0\right\}$. We then have $\mathscr{C}=\left\{\left(x_{1}, x_{2}\right) \mid x_{2}=0,-1<x_{1} \leqslant 0\right\}$. We impose essential boundary conditions on $\partial_{d} \Omega=\partial \bar{\Omega}$ given by $\boldsymbol{g}=\boldsymbol{u}^{e}$. On $\mathscr{C}=\partial_{\tau} \Omega$, we apply the tractions associated with the uniform pressure field, namely, $\boldsymbol{t}=\mathrm{p} \boldsymbol{n}$. In Figure 6, we show a sketch of the modeled problem with boundary conditions alongside the subdivision $\mathcal{T}^{h}$ of $\hat{\Omega}$ and the same mesh mapped with $\gamma$. Note that, as $\Omega=\hat{\Omega}$, we use the same subdivision $\mathcal{T}^{h}$ for both the MFEM and the FEM. The radius of support of the cutoff function is taken as $\rho=1$. We assume a plain strain state and the material constants are $\Lambda=1.5$ and $\mu=1$.

We investigated the $h$-convergence for $\hat{\mathcal{V}}^{h, p}, p=1,2,3,4$, by uniformly subdividing each triangle in the mesh into four similar ones. We showcase in Figure 7(a) a comparison of the convergence of the MFEM against the FEM. The error and the rates of convergence are provided in Table I. We observe optimal convergence rates for the MFEM and sub-optimal rates for FEM. In Figure 7, we also provide comparison of the errors as functions of the problem size $N$ and CPU time. It appears

\begin{tabular}{|c|c|c|c|c|c|c|c|c|}
\hline \multirow[b]{2}{*}{$h_{0} / h$} & \multicolumn{2}{|c|}{$P^{1}$} & \multicolumn{2}{|c|}{$P^{2}$} & \multicolumn{2}{|c|}{$P^{3}$} & \multicolumn{2}{|c|}{$P^{4}$} \\
\hline & Error & $\mathcal{O}$ & Error & $\mathcal{O}$ & Error & $\mathcal{O}$ & Error & $\mathcal{O}$ \\
\hline
\end{tabular}

Table I. Errors $\left(\left\|\boldsymbol{u}^{e}-\boldsymbol{u}^{h}\right\|_{0, \Omega}\right)$ and convergence rates for the straight crack problem.

\begin{tabular}{|c|c|c|c|c|c|c|c|c|}
\hline 1 & $9.1 \cdot 10^{-4}$ & - & $2.6 \cdot 10^{-4}$ & - & $1.3 \cdot 10^{-4}$ & - & $7.5 \cdot 10^{-5}$ & - \\
\hline 2 & $4.5 \cdot 10^{-4}$ & 1.0 & $1.3 \cdot 10^{-4}$ & 1.1 & $6.1 \cdot 10^{-5}$ & 1.0 & $3.7 \cdot 10^{-5}$ & 1.0 \\
\hline 4 & $2.2 \cdot 10^{-4}$ & 1.0 & $6.1 \cdot 10^{-5}$ & 1.0 & $3.0 \cdot 10^{-5}$ & 1.0 & $1.8 \cdot 10^{-5}$ & 1.0 \\
\hline 8 & $1.1 \cdot 10^{-4}$ & 1.0 & $3.0 \cdot 10^{-5}$ & 1.0 & $1.5 \cdot 10^{-5}$ & 1.0 & $9.0 \cdot 10^{-6}$ & 1.0 \\
\hline 16 & $5.5 \cdot 10^{-5}$ & 1.0 & $1.5 \cdot 10^{-5}$ & 1.0 & $7.5 \cdot 10^{-6}$ & 1.0 & - & - \\
\hline 32 & $2.8 \cdot 10^{-5}$ & 1.0 & $7.5 \cdot 10^{-6}$ & 1.0 & - & - & - & 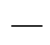 \\
\hline 64 & $1.4 \cdot 10^{-5}$ & 1.0 & - & - & - & - & - & - \\
\hline \multicolumn{9}{|c|}{ (b) MFEM } \\
\hline 1 & $1.8 \cdot 10^{-3}$ & - & $2.8 \cdot 10^{-4}$ & - & $9.1 \cdot 10^{-5}$ & - & $5.7 \cdot 10^{-5}$ & - \\
\hline 2 & $5.4 \cdot 10^{-4}$ & 1.7 & $3.7 \cdot 10^{-5}$ & 2.9 & $5.9 \cdot 10^{-6}$ & 3.9 & $2.5 \cdot 10^{-6}$ & 4.5 \\
\hline 4 & $1.5 \cdot 10^{-4}$ & 1.8 & $4.3 \cdot 10^{-6}$ & 3.1 & $3.9 \cdot 10^{-7}$ & 3.9 & $6.0 \cdot 10^{-8}$ & 5.4 \\
\hline 8 & $4.1 \cdot 10^{-5}$ & 1.9 & $5.3 \cdot 10^{-7}$ & 3.0 & $2.4 \cdot 10^{-8}$ & 4.0 & $1.9 \cdot 10^{-9}$ & 5.0 \\
\hline 16 & $1.0 \cdot 10^{-5}$ & 2.0 & $6.5 \cdot 10^{-8}$ & 3.0 & $1.5 \cdot 10^{-9}$ & 4.0 & - & - \\
\hline 32 & $2.6 \cdot 10^{-6}$ & 2.0 & $8.1 \cdot 10^{-9}$ & 3.0 & - & - & - & - \\
\hline 64 & $6.5 \cdot 10^{-7}$ & 2.0 & - & - & - & - & - & 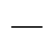 \\
\hline
\end{tabular}

(a) FEM 


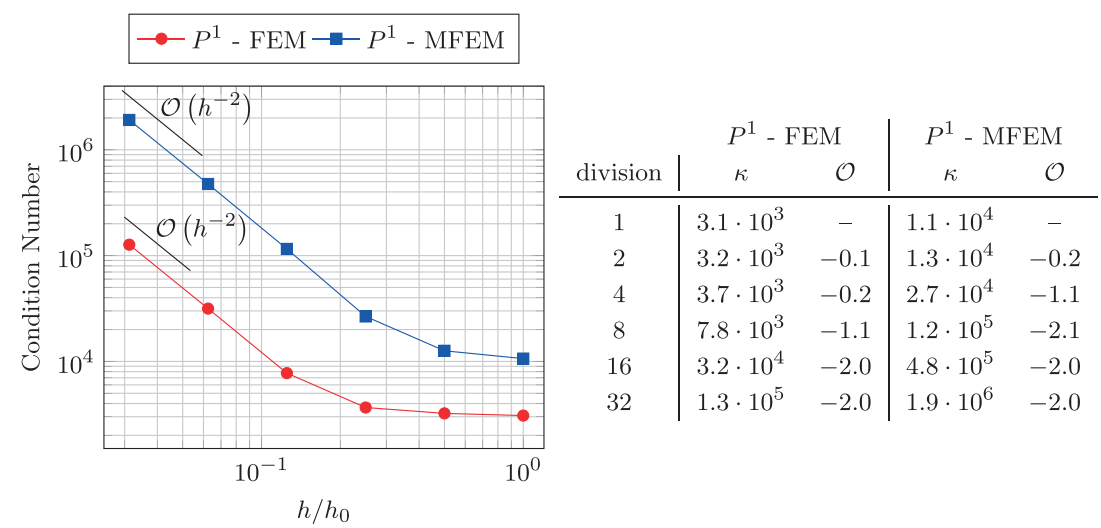

Figure 8. Scaling of the condition number $\kappa$ for the straight crack problem.

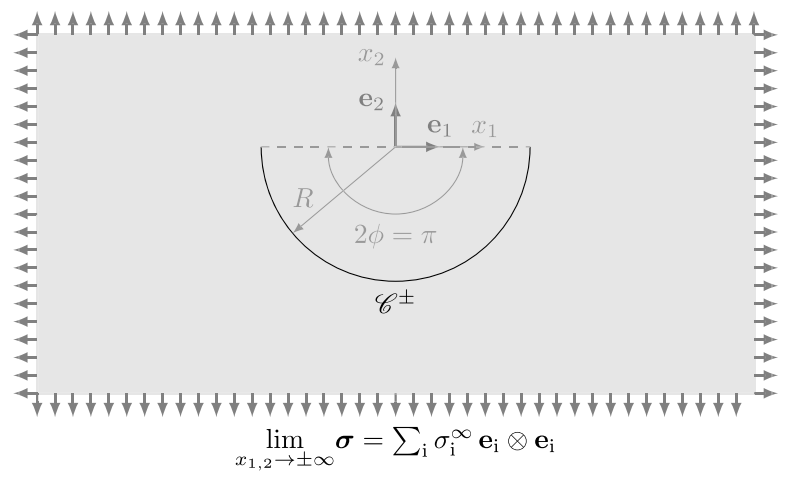

(a) The circular arc crack problem

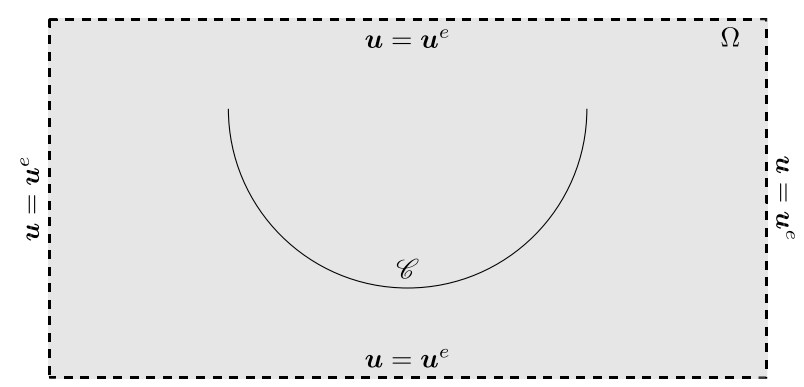

(b) Modeled subdomain with boundary conditions

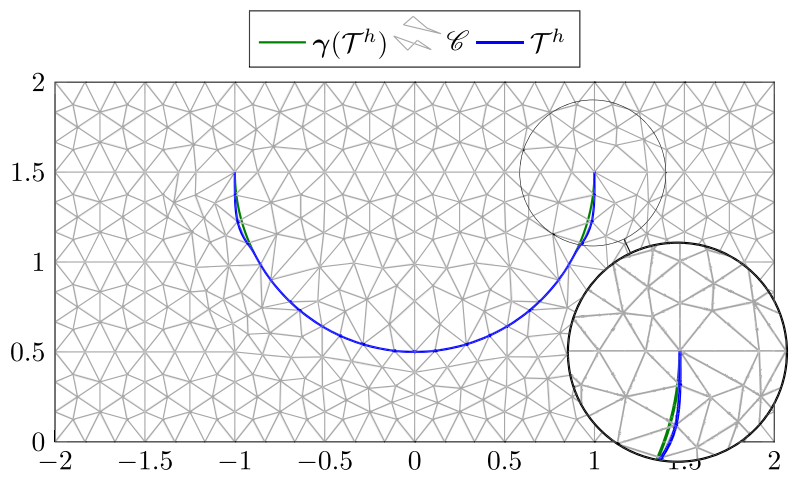

(c) Sample mesh corresponding to the first subdivision

Figure 9. Circular arc crack problem. (a) The circular arc crack problem; (b) modeled subdomain with boundary conditions; (c) sample mesh corresponding to the first subdivision. 


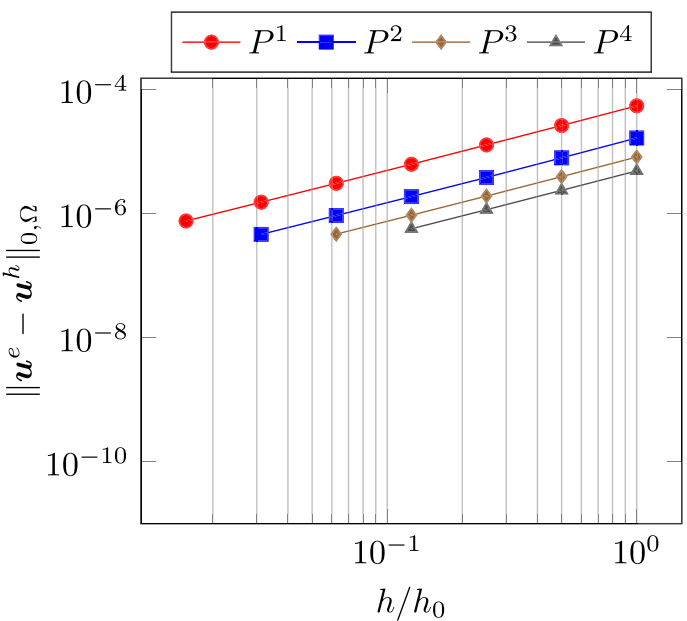

(a) Convergence in mesh size for FEM

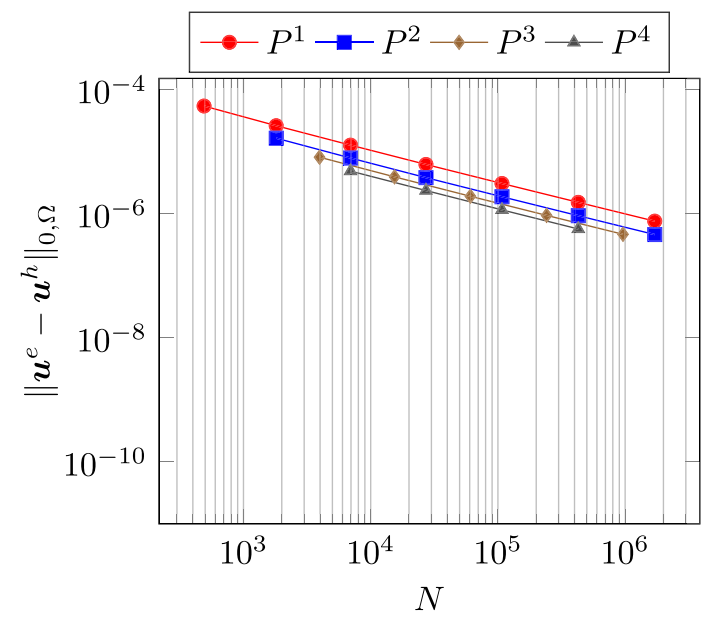

(c) Convergence in system size for FEM

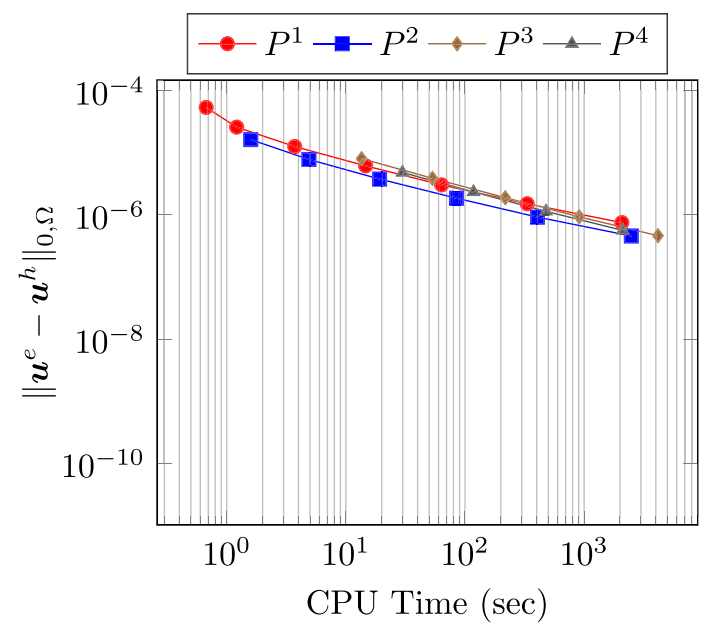

(e) Convergence in CPU time for FEM

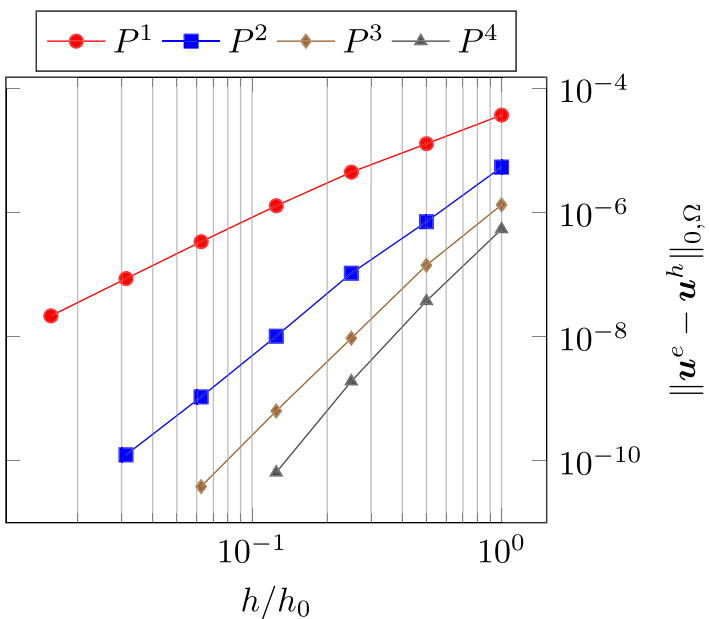

(b) Convergence in mesh size for MFEM

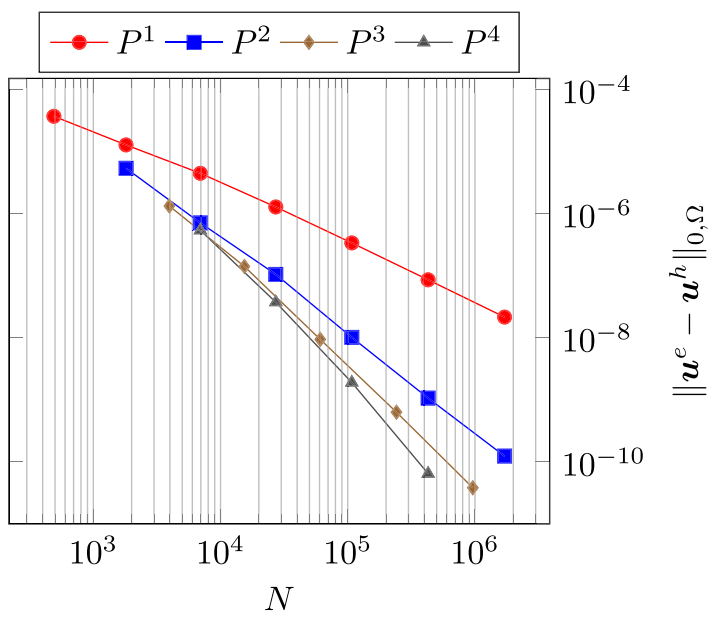

(d) Convergence in system size for MFEM

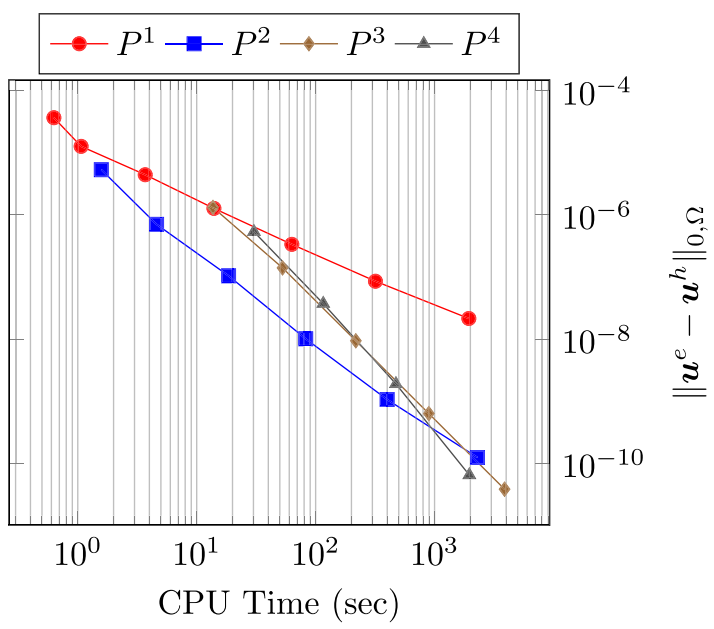

(f) Convergence in CPU time for MFEM

Figure 10. Convergence of the elasticity solution for a circular arc crack. (a) Convergence in mesh size for FEM; (b) convergence in mesh size for MFEM; (c) convergence in system size for FEM; (d) convergence in system size for MFEM; (e) convergence in CPU time for FEM; (f) convergence in CPU time for MFEM. 
that the choice of $p=2$ best balances computing time and error. Notice that with $p=2$ the computing times for an error of $10^{-5}$ differ by around two orders of magnitude, in favor of the MFEM.

One of the key features that unleashes the computational time savings for the MFEM is that, for the auxiliary map presented here, the condition number of the resulting system of equations maintains the same scaling with respect to mesh refinements than standard finite element methods. This is in contrast with graded mesh refinement strategies without proper scaling of the basis functions. More precisely, the condition number of the method was observed to scale as $\mathcal{O}\left(h^{-2}\right)$, just as in standard finite element methods, compare with [32, Chapter 9]. Figure 8 shows the aforementioned behavior. This behavior arises because the bilinear form of the new problem is coercive and continuous with continuity and coercivity constants independent of $h$. Crucial to this fact is that the map itself is defined independently of $h$.

\subsection{Circular arc crack}

We now consider the problem of a circular arc crack in an infinite medium loaded by remote stress, as shown in Figure 9(a). An analytical solution for the problem is available in Muskhelishvili [33], and a Python[34] implementation of the solution is available as part of the Supporting Information in [35]. The objective of the example is to showcase the method in the context of multiple crack tips and curved cracks.

The values of $\sigma_{i}^{\infty}, i=1,2$, were chosen such that mode I stress intensity factor is unity $\left(K_{I}=1\right)$ and mode II stress intensity factor is exactly zero $\left(K_{I I}=0\right)$. The radius of the crack is taken as $R=1$, and the angle subdued by the crack is taken as $2 \phi=\pi$. In Figure 9(b) and (c), we show a sketch of the modeled subdomain given by $\Omega=[-2,2] \times[-1,1]$ with boundary conditions and the coarsest mesh, respectively. The radius of support of the cutoff functions for both crack tips was chosen as $\rho_{\tau}=0.5, \tau=1,2$. Lastly, we assumed a plane strain state with material constants $\Lambda=1.5$, $\mu=1$.

We investigated the $h$-convergence for $\hat{\mathcal{V}}^{h, p}, p=1,2,3,4$. We showcase in Figure 10 a comparison of the convergence of the MFEM against the FEM. The error and the rates of convergence are provided in Table II. As in the previous section, we observe optimal convergence rates for the MFEM and sub-optimal convergence for the FEM.

Table II. Errors $\left(\left\|\boldsymbol{u}^{e}-\boldsymbol{u}^{h}\right\|_{0, \Omega}\right)$ and convergence rates for the circular arc crack problem.

\begin{tabular}{|c|c|c|c|c|c|c|c|c|}
\hline \multirow[b]{2}{*}{$h_{0} / h$} & \multicolumn{2}{|l|}{$P^{1}$} & \multicolumn{2}{|l|}{$P^{2}$} & \multicolumn{2}{|l|}{$P^{3}$} & \multicolumn{2}{|l|}{$P^{4}$} \\
\hline & Error & $\mathcal{O}$ & Error & $\mathcal{O}$ & Error & $\mathcal{O}$ & Error & $\mathcal{O}$ \\
\hline \multicolumn{9}{|c|}{ (a) FEM } \\
\hline 1 & $5.4 \cdot 10^{-5}$ & - & $1.6 \cdot 10^{-5}$ & - & $8.1 \cdot 10^{-6}$ & - & $4.8 \cdot 10^{-6}$ & - \\
\hline 2 & $2.6 \cdot 10^{-5}$ & 1.1 & $7.8 \cdot 10^{-6}$ & 1.1 & $3.9 \cdot 10^{-6}$ & 1.1 & $2.3 \cdot 10^{-6}$ & 1.0 \\
\hline 4 & $1.3 \cdot 10^{-5}$ & 1.0 & $3.8 \cdot 10^{-6}$ & 1.1 & $1.9 \cdot 10^{-6}$ & 1.0 & $1.1 \cdot 10^{-6}$ & 1.0 \\
\hline 8 & $6.2 \cdot 10^{-6}$ & 1.0 & $1.9 \cdot 10^{-6}$ & 1.0 & $9.3 \cdot 10^{-7}$ & 1.0 & $5.6 \cdot 10^{-7}$ & 1.0 \\
\hline 16 & $3.0 \cdot 10^{-6}$ & 1.0 & $9.2 \cdot 10^{-7}$ & 1.0 & $4.6 \cdot 10^{-7}$ & 1.0 & - & - \\
\hline 32 & $1.5 \cdot 10^{-6}$ & 1.0 & $4.6 \cdot 10^{-7}$ & 1.0 & - & - & - & - \\
\hline 64 & $7.5 \cdot 10^{-7}$ & 1.0 & - & - & - & - & - & - \\
\hline \multicolumn{9}{|c|}{ (b) MFEM } \\
\hline 1 & $3.7 \cdot 10^{-5}$ & - & $5.4 \cdot 10^{-6}$ & - & $1.3 \cdot 10^{-6}$ & - & $5.4 \cdot 10^{-7}$ & - \\
\hline 2 & $1.3 \cdot 10^{-5}$ & 1.5 & $7.1 \cdot 10^{-7}$ & 2.9 & $1.4 \cdot 10^{-7}$ & 3.2 & $3.7 \cdot 10^{-8}$ & 3.8 \\
\hline 4 & $4.4 \cdot 10^{-6}$ & 1.5 & $1.0 \cdot 10^{-7}$ & 2.8 & $9.4 \cdot 10^{-9}$ & 3.9 & $1.9 \cdot 10^{-9}$ & 4.3 \\
\hline 8 & $1.3 \cdot 10^{-6}$ & 1.8 & $1.0 \cdot 10^{-8}$ & 3.4 & $6.3 \cdot 10^{-10}$ & 3.9 & $6.4 \cdot 10^{-11}$ & 4.9 \\
\hline 16 & $3.4 \cdot 10^{-7}$ & 1.9 & $1.1 \cdot 10^{-9}$ & 3.2 & $3.8 \cdot 10^{-11}$ & 4.0 & - & - \\
\hline 32 & $8.5 \cdot 10^{-8}$ & 2.0 & $1.2 \cdot 10^{-10}$ & 3.1 & - & - & - & - \\
\hline 64 & $2.1 \cdot 10^{-8}$ & 2.0 & - & - & - & - & - & - \\
\hline
\end{tabular}




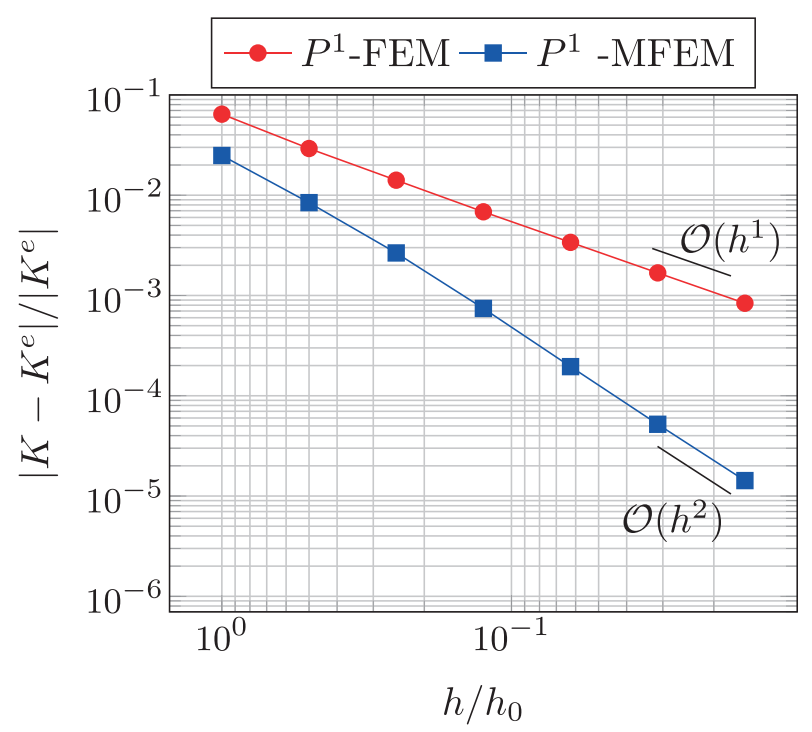

Figure 11. Convergence of the stress intensity factors for a circular arc crack. We highlight that the rate of convergence of the stress intensity factors is twice the rates of convergence of the derivatives of the solution (where $\left\|\nabla \boldsymbol{u}^{h}-\nabla \boldsymbol{u}^{e}\right\|_{0, \Omega} \leqslant \mathcal{O}\left(h^{k}\right)$ with $k=0.5$ for FEM and $k=p$ for MFEM, cf. Section 4.4, Figure 16, and Table V).
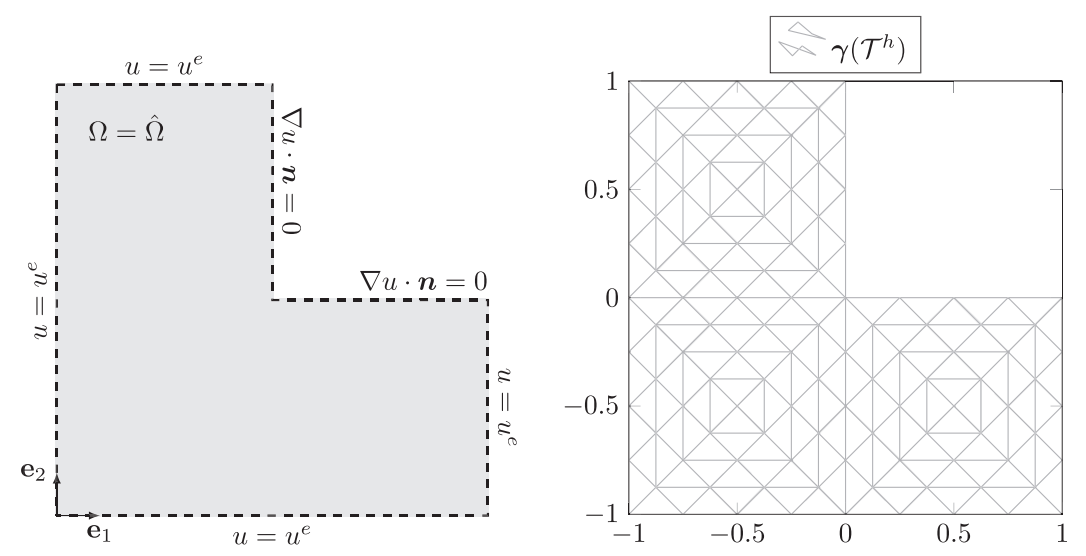

(a) Modeled subdomain with boundary conditions (b) Sample mesh corresponding to the first subdivision

Figure 12. Anti-plane elasticity over an L-shaped domain. (a) Modeled subdomain with boundary conditions; (b) sample mesh corresponding to the first subdivision.

The importance of the optimality in the convergence of the derivative becomes apparent, for example, when considering simulations of crack propagation. The evolution of a crack is dictated by two scalar coefficients known as the stress intensity factors (SIFs). The SIFs can be computed by evaluating a continuous linear functional of the derivative of the computed solution (e.g., [36]) known as the interaction integral functional. It can be shown that such functionals converge at twice the rate of their argument. Namely, if $I[\cdot]$ denotes the Interaction Integral functional, then we have $\left\|I\left[\nabla \boldsymbol{u}^{e}\right]-I\left[\nabla \boldsymbol{u}^{h}\right]\right\| \leqslant \mathcal{O}\left(h^{2 k}\right)$ where $\left\|\boldsymbol{u}^{e}-\boldsymbol{u}^{h}\right\|_{1, \Omega} \leqslant \mathcal{O}\left(h^{k}\right)$. This behavior is indeed observed in the evaluation of the SIFs for a circular arc crack and illustrated in Figure 11, where we contrast the convergence of the SIFs from a solution obtained using FEM with that of using MFEM. In the former $k=0.5$, and hence the SIFs converge as $\mathcal{O}\left(h^{1}\right)$, while in the latter $k=p$, and hence the SIFs converge as $\mathcal{O}\left(h^{2 p}\right)$. Convergence curves for the derivatives are provided for a different example in Section 4.4. 


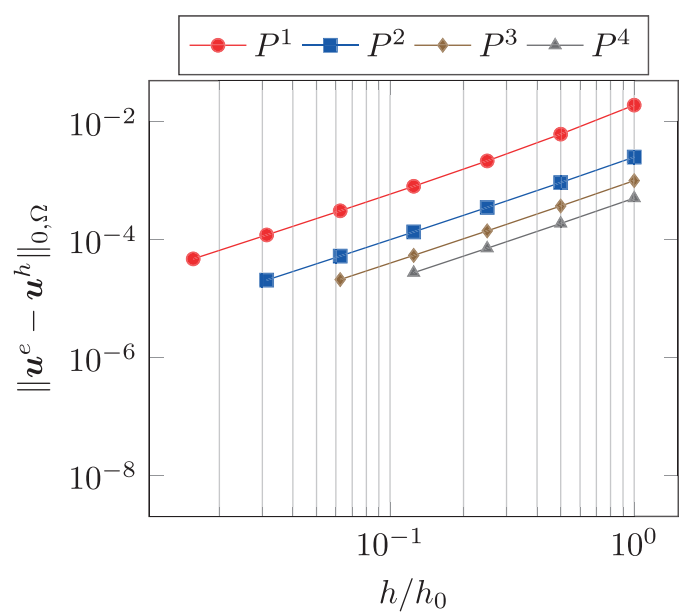

(a) Convergence in mesh size for FEM

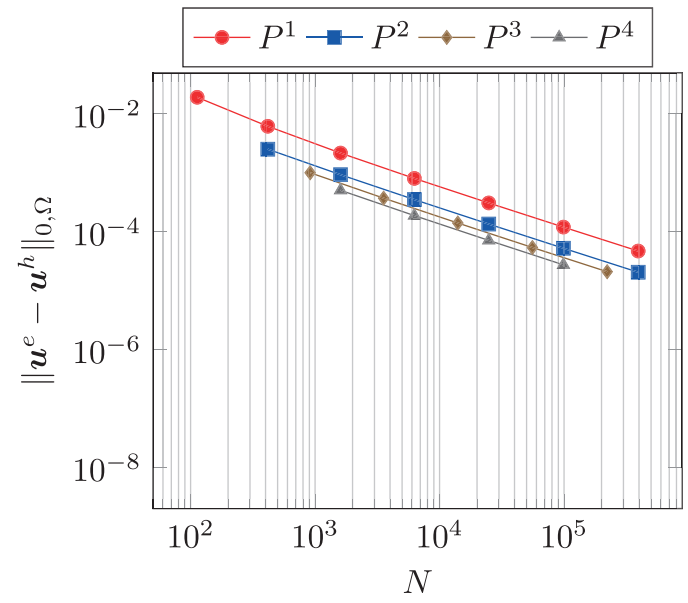

(c) Convergence in system size for FEM

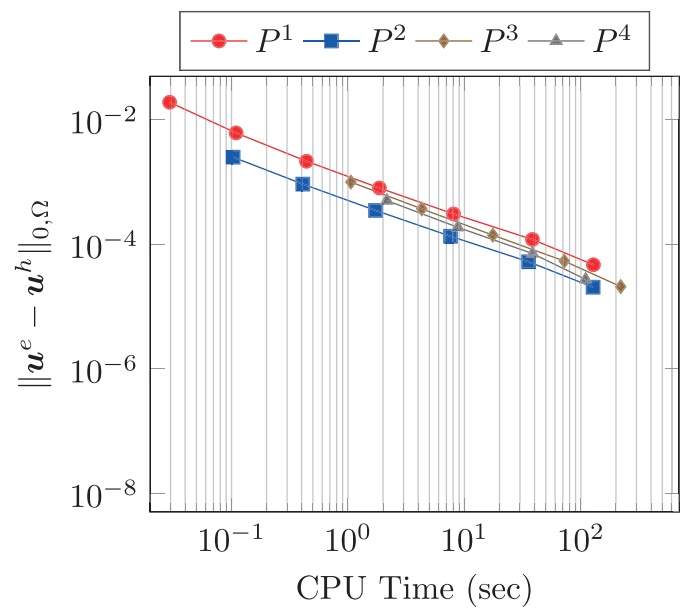

(e) Convergence in CPU time for FEM

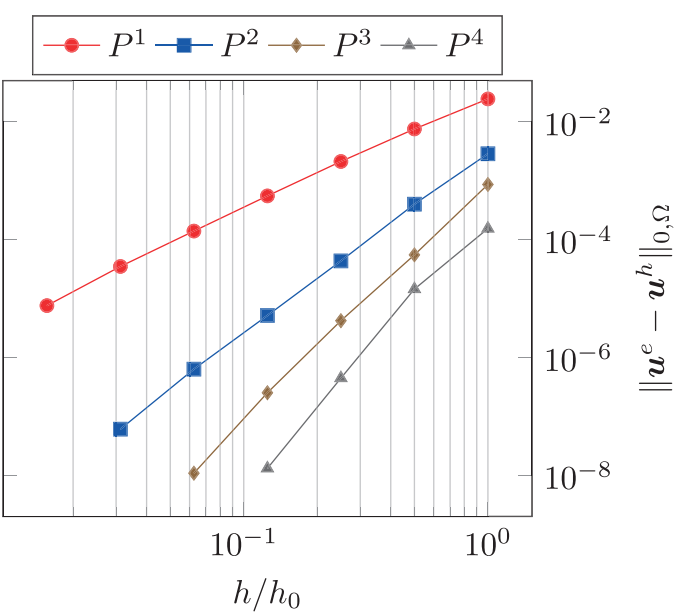

(b) Convergence in mesh size for MFEM

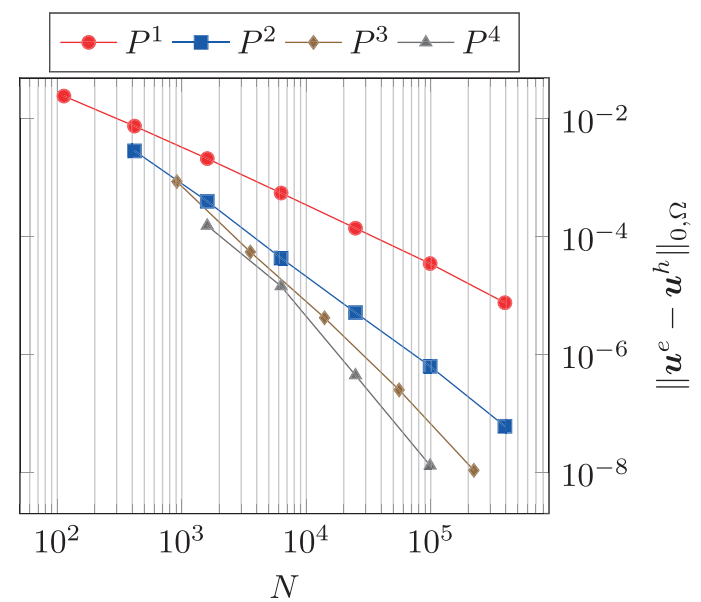

(d) Convergence in system size for MFEM

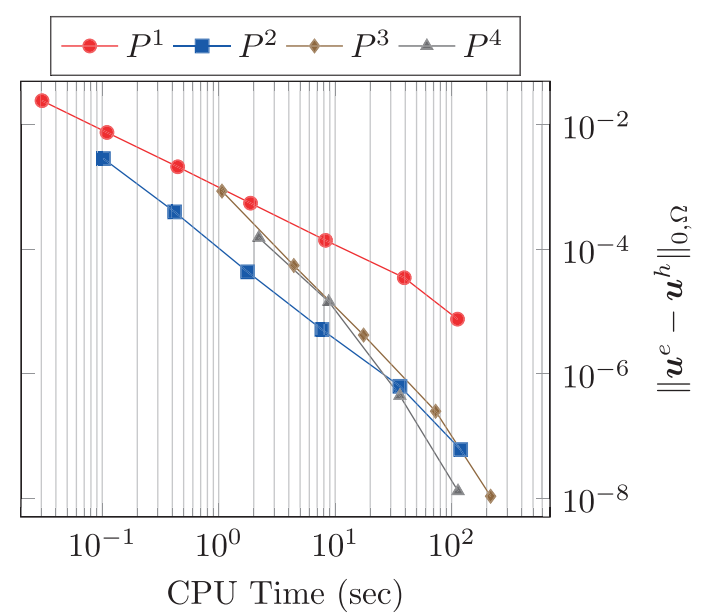

(f) Convergence in CPU time for MFEM

Figure 13. Convergence of the anti-plane elasticity solution for an L-shaped domain. (a) Convergence in mesh size for FEM; (b) convergence in mesh size for MFEM; (c) convergence in system size for FEM; (d) convergence in system size for MFEM; (e) convergence in CPU time for FEM; (f) convergence in CPU time for MFEM. 


\subsection{Anti-plane elasticity over an L-shaped domain}

The next example we present is aimed at showcasing the versatility of the MFEM for problems with re-entrant corners. The problem of interest is the anti-plane deformation of an L-shaped domain, as shown in Figure 12. As we remarked earlier in the manuscript, the boundary value problem becomes solving $u: \Omega \rightarrow \mathbb{R}$ such that

$$
\begin{aligned}
\nabla \cdot(\mu \nabla u)+b & =0, & & \text { in } \Omega, \\
u & =g, & & \text { on } \partial_{d} \Omega, \\
\mu \nabla u \cdot \boldsymbol{n} & =t, & & \text { on } \partial_{\tau} \Omega .
\end{aligned}
$$

It is rather straightforward to follow the steps of Section 3 to derive the Garlerkin form over $\hat{\Omega}$ of the aforementioned boundary value problem. We provide details of the bilinear form and other relevant constructs in Appendix C.

The domain is $\Omega=(-1,1)^{2} \backslash[0,1]^{2}$. We then let $\partial_{d} \Omega=\partial \Omega \backslash[(\{0\} \times[0,1]) \cup([0,1] \times\{0\})]$. We take $t=0, b=2\left(x_{1}^{2}+x_{2}^{2}\right)$ and set

$$
g=r^{2 / 3} \cos (2 \theta / 3)+x_{1}^{2} x_{2}^{2}
$$

where the $x_{1}$-axis coincides with the ray $\theta=0$. The solution to the aforementioned boundary value problem is given by

$$
u^{e}=r^{2 / 3} \cos (2 \theta / 3)+x_{1}^{2} x_{2}^{2} .
$$

The power of the singularity is $2 / 3$ rather than $1 / 2$ as in the case of a cracked domain. We could very well replace the condition $m \geqslant k \geqslant p+1$ by a milder one and still maintain $u^{e} \circ \gamma \in H^{k}(\hat{\Omega})$ and recover optimal rates of convergence. Alternatively, given that the solution to the L-shaped domain is less singular than that of the cracked domain, it will not be necessary to alter the method at all. In fact using a mapping $\gamma$ given by (11) with the original condition $m \geqslant k \geqslant p+1$ will yield an even smoother solution. Therefore, for the example, the mapping remains the same as (11).

The radius of the cutoff function was taken as $\rho=1$, and the material parameters were chosen once more as $\mu=1$.

We provide convergence plots for four polynomial degrees in Figure 13. Once again, alongside the convergence in the mesh size, we showcase comparisons of error evolution as a function of the problem size and computing time. It is worthwhile noting that for this particular example the solution of FEM converges faster than for the cracked domain, but still suboptimally, namely, $\left\|u^{e}-u^{h}\right\| \leqslant$ $\mathcal{O}\left(h^{4 / 3}\right)$. The above is attributed to the higher regularity of the solution, namely, $u^{e} \in H^{5 / 3-\delta}(\Omega)$ for any $\delta>0$ [37].

Consistent with what was observed before, the solution computed with MFEM converges optimally. Table III shows the error and the computed rates of convergence. Once more quadratic polynomial interpolates provide the best compromise between run-time and accuracy for the range of errors here presented.

\subsection{Interface crack}

The last problem we present is the one of a crack along a bilateral interface. The material properties are chosen such that $\Lambda_{1}=1.5, \mu_{1}=1, \Lambda_{2}=10 \Lambda_{1}$, and $\mu_{2}=10 \mu_{1}$. The analytical solution for the problem can be found in [38]. The objective of the example is to showcase the ability of the same method to obtain higher-order approximations of the elasticity fields in the presence of different asymptotic behaviors. More precisely, the displacement field in medium $M, M=1,2$, scales as

$$
\boldsymbol{u} \sim r^{1 / 2}\left[\boldsymbol{\psi}_{M a}(\theta) \cos (\varepsilon \ln r)+\boldsymbol{\psi}_{M b}(\theta) \sin (\varepsilon \ln r)\right],
$$

where $\boldsymbol{\psi}_{M a}$ and $\boldsymbol{\psi}_{M b}$ are $C^{\infty}$ functions of $\theta$, and $\varepsilon$ depends on the elastic constants of the two materials, which vanishes if $\Lambda_{1}=\Lambda_{2}$ and $\mu_{1}=\mu_{2}$. In this example, $\varepsilon=18 / 77 \doteq 0.2338$. This asymptotic behavior in the physical domain leads to, in the mapped domain with $m=k$, 
Table III. Errors $\left(\left\|\boldsymbol{u}^{e}-\boldsymbol{u}^{h}\right\|_{0, \Omega}\right)$ and convergence rates for the anti-plane elasticity problem on and L-shaped domain.

\begin{tabular}{|c|c|c|c|c|c|c|c|c|}
\hline \multirow[b]{2}{*}{$h_{0} / h$} & \multicolumn{2}{|c|}{$P^{1}$} & \multicolumn{2}{|c|}{$P^{2}$} & \multicolumn{2}{|c|}{$P^{3}$} & \multicolumn{2}{|c|}{$P^{4}$} \\
\hline & Error & $\mathcal{O}$ & Error & $\mathcal{O}$ & Error & $\mathcal{O}$ & Error & $\mathcal{O}$ \\
\hline
\end{tabular}

(a) FEM

\begin{tabular}{lcccccccc}
1 & $1.9 \cdot 10^{-2}$ & - & $2.5 \cdot 10^{-3}$ & - & $1.0 \cdot 10^{-3}$ & - & $5.1 \cdot 10^{-4}$ & - \\
2 & $6.2 \cdot 10^{-3}$ & 1.6 & $9.3 \cdot 10^{-4}$ & 1.4 & $3.7 \cdot 10^{-4}$ & 1.4 & $1.9 \cdot 10^{-4}$ & 1.4 \\
4 & $2.2 \cdot 10^{-3}$ & 1.5 & $3.5 \cdot 10^{-4}$ & 1.4 & $1.4 \cdot 10^{-4}$ & 1.4 & $7.1 \cdot 10^{-5}$ & 1.4 \\
8 & $8.0 \cdot 10^{-4}$ & 1.4 & $1.4 \cdot 10^{-4}$ & 1.4 & $5.4 \cdot 10^{-5}$ & 1.4 & $2.7 \cdot 10^{-5}$ & 1.4 \\
16 & $3.1 \cdot 10^{-4}$ & 1.4 & $5.2 \cdot 10^{-5}$ & 1.4 & $2.1 \cdot 10^{-5}$ & 1.4 & - & - \\
32 & $1.2 \cdot 10^{-4}$ & 1.4 & $2.1 \cdot 10^{-5}$ & 1.4 & - & - & - & - \\
64 & $4.7 \cdot 10^{-5}$ & 1.4 & - & - & - & - & - & - \\
\multicolumn{7}{c}{} & \multicolumn{7}{c}{ (b) MFEM } & & & \\
1 & $2.4 \cdot 10^{-2}$ & - & $2.9 \cdot 10^{-3}$ & - & $8.6 \cdot 10^{-4}$ & - & $1.5 \cdot 10^{-4}$ & - \\
2 & $7.5 \cdot 10^{-3}$ & 1.7 & $4.0 \cdot 10^{-4}$ & 2.8 & $5.5 \cdot 10^{-5}$ & 4.0 & $1.4 \cdot 10^{-5}$ & 3.4 \\
4 & $2.1 \cdot 10^{-3}$ & 1.8 & $4.3 \cdot 10^{-5}$ & 3.2 & $4.2 \cdot 10^{-6}$ & 3.7 & $4.5 \cdot 10^{-7}$ & 5.0 \\
8 & $5.5 \cdot 10^{-4}$ & 1.9 & $5.2 \cdot 10^{-6}$ & 3.1 & $2.5 \cdot 10^{-7}$ & 4.1 & $1.3 \cdot 10^{-8}$ & 5.1 \\
16 & $1.4 \cdot 10^{-4}$ & 2.0 & $6.3 \cdot 10^{-7}$ & 3.0 & $1.1 \cdot 10^{-8}$ & 4.5 & - & - \\
32 & $3.5 \cdot 10^{-5}$ & 2.0 & $6.1 \cdot 10^{-8}$ & 3.4 & - & - & - & - \\
64 & $7.6 \cdot 10^{-6}$ & 2.2 & - & - & - & - & - & - \\
\hline
\end{tabular}

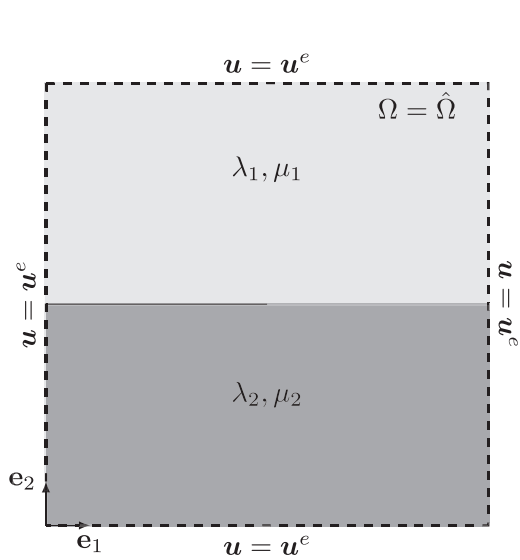

(a) Modeled subdomain with boundary conditions

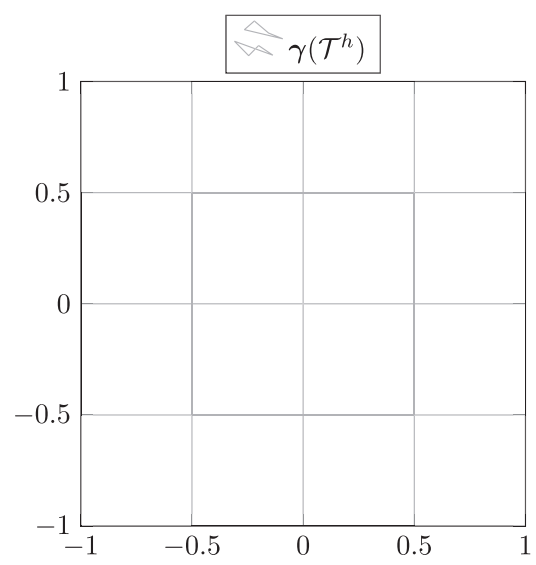

(b) Coarsest subdivision

Figure 14. Interface crack. (a) Modeled subdomain with boundary conditions; (b) coarsest subdivision.

$$
\hat{\boldsymbol{u}} \sim \hat{r}^{k}\left[\hat{\boldsymbol{\psi}}_{M a}(\hat{\theta}) \cos (2 k \varepsilon \ln \hat{r})+\hat{\boldsymbol{\psi}}_{M b}(\hat{\theta}) \sin (2 k \varepsilon \ln \hat{r})\right] \in H^{k}\left(\hat{\Omega}, \mathbb{R}^{2}\right),
$$

where $\hat{\boldsymbol{\psi}}_{M a}$ and $\hat{\boldsymbol{\psi}}_{M b}$ are $C^{\infty}$ functions of $\hat{\theta}$. Hence, no alteration is needed to apply the method to this problem to achieve optimal convergence.

In comparison, in the case of extended finite element methods (XFEM), basis functions that span the different types of singularities need to be employed to attain at most first-order convergence in the stresses. An example of the latter, albeit with stresses converging as $h^{1 / 2}$, can be found in [39].

As the analytical solutions are available, we modeled a subdomain $\Omega=(-1,1)^{2} \backslash([-1,0] \times\{0\})$ containing only a single crack tip and prescribed displacements on the boundary of the domain, as illustrated in Figure 14(a). The coarsest mesh is shown in in Figure 14(b); and each mesh was 


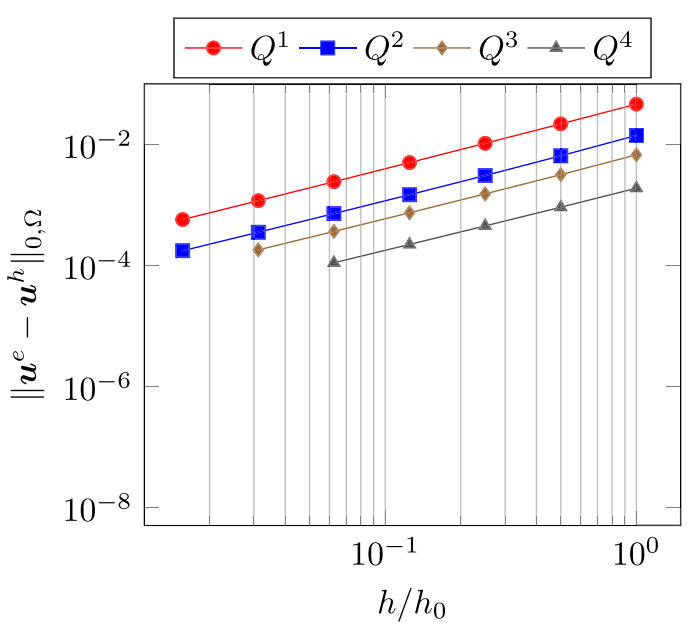

(a) Convergence in mesh size for FEM

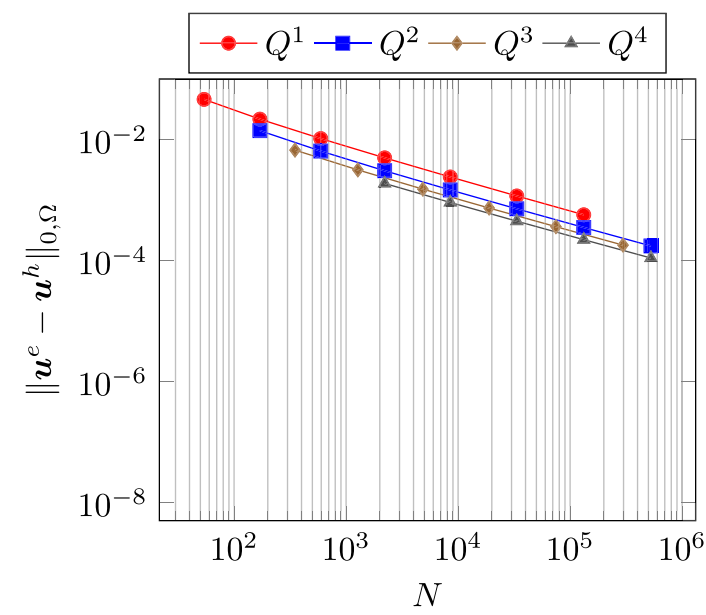

(c) Convergence in system size for FEM

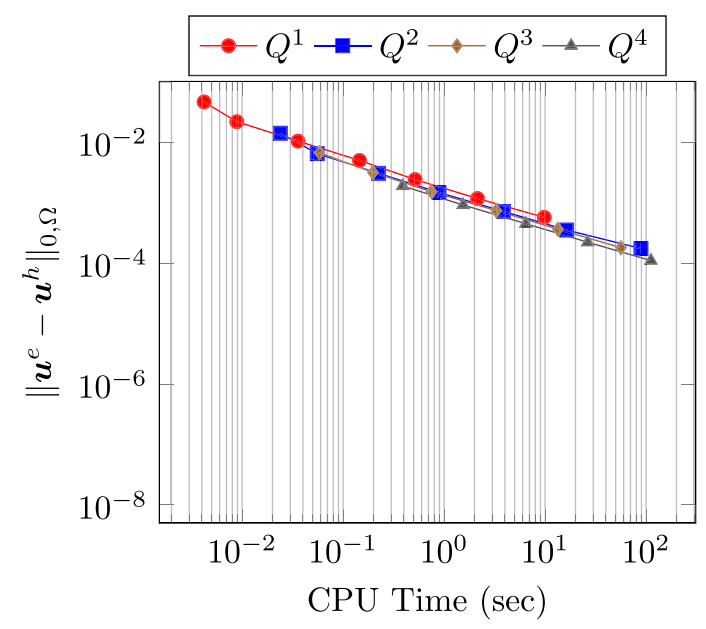

(e) Convergence in CPU time for FEM

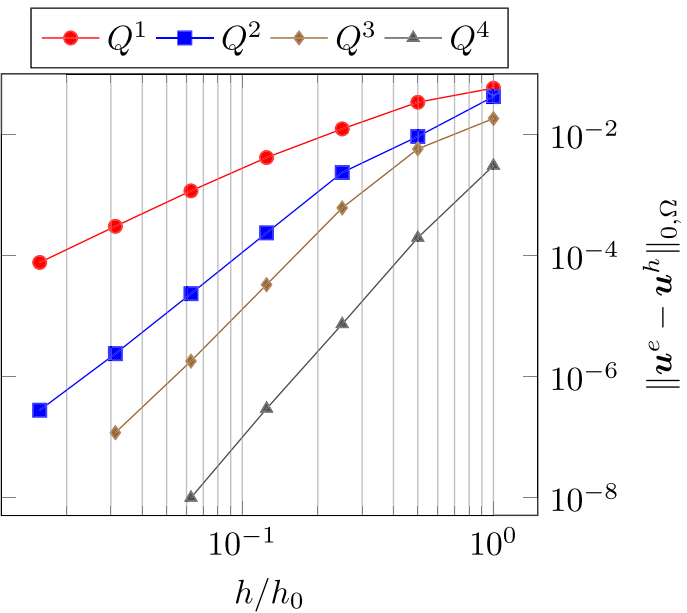

(b) Convergence in mesh size for MFEM

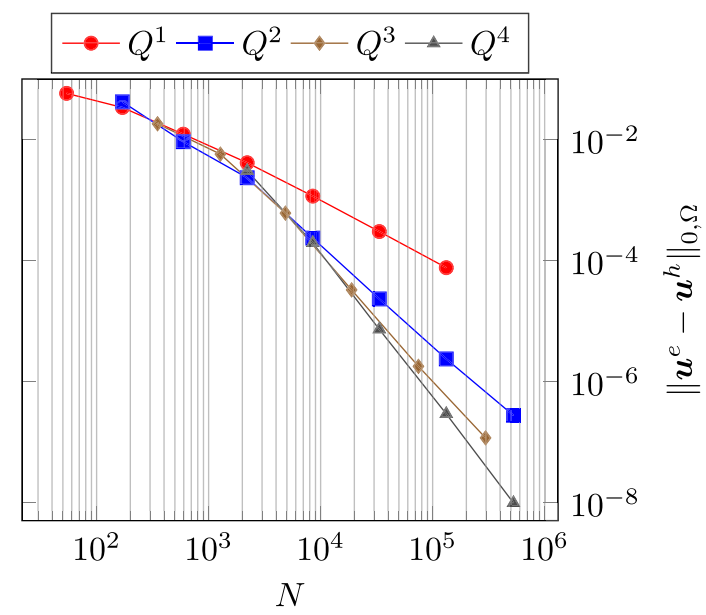

(d) Convergence in system size for MFEM

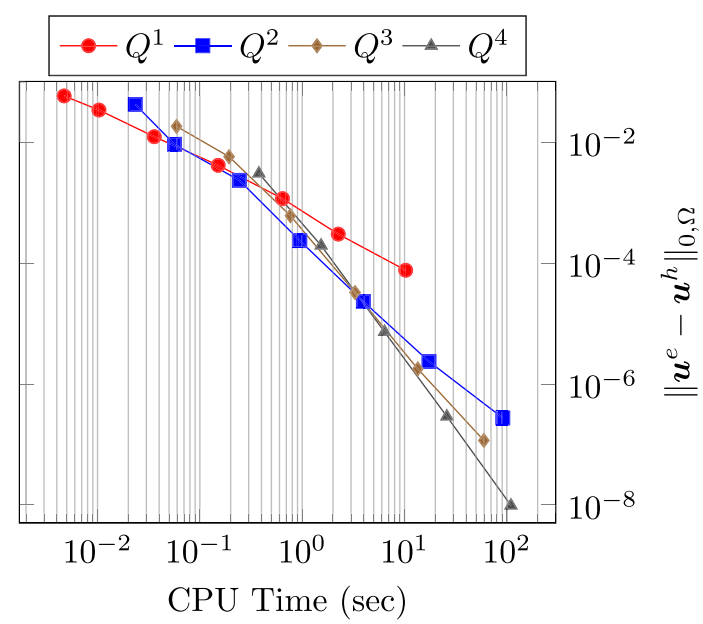

(f) Convergence in CPU time for MFEM

Figure 15. Convergence of the solution of the elasticity field of a crack at a bimaterial interface. (a) Convergence in mesh size for FEM; (b) convergence in mesh size for MFEM; (c) convergence in system size for FEM; (d) convergence in system size for MFEM; (e) convergence in CPU time for FEM; (f) Convergence in CPU time for MFEM. 
obtained by recursive subdivisions of the coarsest mesh. We remark that for this example we employed quadrilateral elements $Q^{k}$ to showcase the independence of the method on the type of finite element. The radius of support of the mapping is $\rho=1$. As before, we performed an $h$-convergence study for four degrees of the polynomial interpolate ranging from linear to quartic $\left(Q^{k}, k=1,2,3,4\right)$ in Figure 15. Table IV shows the error and the computed rates of convergence. Results similar to the previous examples are obtained.

We would like to remark that optimality in the convergence of the derivative, alongside the solutions themselves, was also observed. We expect the error in the derivative to converge optimally for MFEM, namely, $\left\|\boldsymbol{u}^{e}-\boldsymbol{u}^{h}\right\|_{1, \Omega} \leqslant \mathcal{O}\left(h^{p}\right)$ with $p$ being the order of the polynomial interpolate, while

Table IV. Errors $\left(\left\|\boldsymbol{u}^{e}-\boldsymbol{u}^{h}\right\|_{0, \Omega}\right)$ and convergence rates of the elasticity field of a crack at a bimaterial interface.

\begin{tabular}{|c|c|c|c|c|c|c|c|c|}
\hline \multirow[b]{2}{*}{$h_{0} / h$} & \multicolumn{2}{|c|}{$Q^{1}$} & \multicolumn{2}{|c|}{$Q^{2}$} & \multicolumn{2}{|c|}{$Q^{3}$} & \multicolumn{2}{|c|}{$Q^{4}$} \\
\hline & Error & $\mathcal{O}$ & Error & $\mathcal{O}$ & Error & $\mathcal{O}$ & Error & $\mathcal{O}$ \\
\hline
\end{tabular}

(a) FEM

\begin{tabular}{lllllllll}
1 & $4.6 \cdot 10^{-2}$ & - & $1.4 \cdot 10^{-2}$ & - & $6.7 \cdot 10^{-3}$ & - & $1.9 \cdot 10^{-3}$ & - \\
2 & $2.2 \cdot 10^{-2}$ & 1.1 & $6.4 \cdot 10^{-3}$ & 1.1 & $3.1 \cdot 10^{-3}$ & 1.1 & $9.1 \cdot 10^{-4}$ & 1.0 \\
4 & $1.0 \cdot 10^{-2}$ & 1.1 & $3.0 \cdot 10^{-3}$ & 1.1 & $1.5 \cdot 10^{-3}$ & 1.1 & $4.5 \cdot 10^{-4}$ & 1.0 \\
8 & $5.0 \cdot 10^{-3}$ & 1.1 & $1.5 \cdot 10^{-3}$ & 1.1 & $7.4 \cdot 10^{-4}$ & 1.0 & $2.2 \cdot 10^{-4}$ & 1.0 \\
16 & $2.4 \cdot 10^{-3}$ & 1.1 & $7.1 \cdot 10^{-4}$ & 1.0 & $3.6 \cdot 10^{-4}$ & 1.0 & $1.1 \cdot 10^{-4}$ & 1.0 \\
32 & $1.2 \cdot 10^{-3}$ & 1.0 & $3.5 \cdot 10^{-4}$ & 1.0 & $1.8 \cdot 10^{-4}$ & 1.0 & - & - \\
64 & $5.7 \cdot 10^{-4}$ & 1.0 & $1.7 \cdot 10^{-4}$ & 1.0 & - & - & - & - \\
& & & \multicolumn{7}{c}{$(\mathrm{b})$} & $\mathrm{MFEM}$ & & & & \\
1 & $5.8 \cdot 10^{-2}$ & - & $4.2 \cdot 10^{-2}$ & - & $1.8 \cdot 10^{-2}$ & - & $3.0 \cdot 10^{-3}$ & - \\
2 & $3.4 \cdot 10^{-2}$ & 0.8 & $9.2 \cdot 10^{-3}$ & 2.2 & $5.7 \cdot 10^{-3}$ & 1.7 & $1.9 \cdot 10^{-4}$ & 4.0 \\
4 & $1.2 \cdot 10^{-2}$ & 1.5 & $2.3 \cdot 10^{-3}$ & 2.0 & $6.1 \cdot 10^{-4}$ & 3.2 & $7.3 \cdot 10^{-6}$ & 4.7 \\
8 & $4.1 \cdot 10^{-3}$ & 1.6 & $2.3 \cdot 10^{-4}$ & 3.3 & $3.3 \cdot 10^{-5}$ & 4.2 & $2.9 \cdot 10^{-7}$ & 4.6 \\
16 & $1.2 \cdot 10^{-3}$ & 1.8 & $2.3 \cdot 10^{-5}$ & 3.3 & $1.8 \cdot 10^{-6}$ & 4.2 & $9.8 \cdot 10^{-9}$ & 4.9 \\
32 & $3.0 \cdot 10^{-4}$ & 1.9 & $2.4 \cdot 10^{-6}$ & 3.3 & $1.2 \cdot 10^{-7}$ & 3.9 & - & - \\
64 & $7.6 \cdot 10^{-5}$ & 2.0 & $2.7 \cdot 10^{-7}$ & 3.1 & - & - & - & - \\
\hline
\end{tabular}

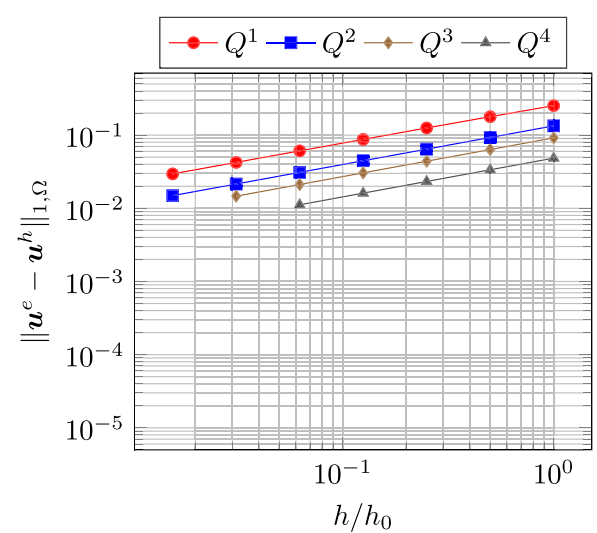

(a) Convergence in mesh size for FEM

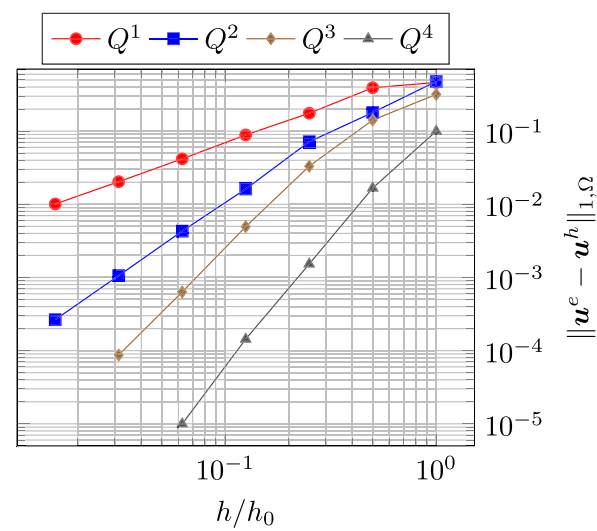

(b) Convergence in mesh size for MFEM

Figure 16. Convergence of the derivatives of the solution. In (a), we see suboptimal rates for FEM, namely, $\left\|\boldsymbol{u}^{e}-\boldsymbol{u}^{h}\right\|_{1, \Omega} \leqslant \mathcal{O}\left(h^{1 / 2}\right)$ independently of the polynomial interpolate, while we recover the optimal rates, $\| \boldsymbol{u}^{e}-$ $\boldsymbol{u}^{h} \|_{1, \Omega} \leqslant \mathcal{O}\left(h^{p+1}\right)$ for MFEM, with $p$ being the degree of the polynomial interpolate. (a) Convergence in mesh size for FEM; (b) convergence in mesh size for MFEM. 
Table V. Errors of the derivative of the solution $\left(\left\|\boldsymbol{u}^{e}-\boldsymbol{u}^{h}\right\|_{1, \Omega}\right)$ and convergence rates for the problem of a crack at a bimaterial interface.

\begin{tabular}{|c|c|c|c|c|c|c|c|c|}
\hline \multirow[b]{2}{*}{$h_{0} / h$} & \multicolumn{2}{|l|}{$Q^{1}$} & \multicolumn{2}{|l|}{$Q^{2}$} & \multicolumn{2}{|l|}{$Q^{3}$} & \multicolumn{2}{|l|}{$Q^{4}$} \\
\hline & Error & $\mathcal{O}$ & Error & $\mathcal{O}$ & Error & $\mathcal{O}$ & Error & $\mathcal{O}$ \\
\hline \multicolumn{9}{|c|}{ (a) FEM } \\
\hline 1 & $2.5 \cdot 10^{-1}$ & - & $1.3 \cdot 10^{-1}$ & - & $9.2 \cdot 10^{-2}$ & - & $4.9 \cdot 10^{-2}$ & - \\
\hline 2 & $1.8 \cdot 10^{-1}$ & 0.5 & $9.3 \cdot 10^{-2}$ & 0.5 & $6.4 \cdot 10^{-2}$ & 0.5 & $3.4 \cdot 10^{-2}$ & 0.5 \\
\hline 4 & $1.3 \cdot 10^{-1}$ & 0.5 & $6.4 \cdot 10^{-2}$ & 0.5 & $4.4 \cdot 10^{-2}$ & 0.5 & $2.3 \cdot 10^{-2}$ & 0.5 \\
\hline 8 & $8.7 \cdot 10^{-2}$ & 0.5 & $4.5 \cdot 10^{-2}$ & 0.5 & $3.1 \cdot 10^{-2}$ & 0.5 & $1.6 \cdot 10^{-2}$ & 0.5 \\
\hline 16 & $6.1 \cdot 10^{-2}$ & 0.5 & $3.1 \cdot 10^{-2}$ & 0.5 & $2.1 \cdot 10^{-2}$ & 0.5 & $1.1 \cdot 10^{-2}$ & 0.5 \\
\hline 32 & $4.2 \cdot 10^{-2}$ & 0.5 & $2.1 \cdot 10^{-2}$ & 0.5 & $1.5 \cdot 10^{-2}$ & 0.5 & - & - \\
\hline 64 & $3.0 \cdot 10^{-2}$ & 0.5 & $1.5 \cdot 10^{-2}$ & 0.5 & - & - & - & - \\
\hline \multicolumn{9}{|c|}{ (b) MFEM } \\
\hline 1 & $4.6 \cdot 10^{-1}$ & - & $4.8 \cdot 10^{-1}$ & - & $3.2 \cdot 10^{-1}$ & - & $1.0 \cdot 10^{-1}$ & - \\
\hline 2 & $3.9 \cdot 10^{-1}$ & 0.2 & $1.8 \cdot 10^{-1}$ & 1.4 & $1.4 \cdot 10^{-1}$ & 1.2 & $1.7 \cdot 10^{-2}$ & 2.6 \\
\hline 4 & $1.8 \cdot 10^{-1}$ & 1.2 & $7.2 \cdot 10^{-2}$ & 1.3 & $3.3 \cdot 10^{-2}$ & 2.1 & $1.5 \cdot 10^{-3}$ & 3.4 \\
\hline 8 & $8.9 \cdot 10^{-2}$ & 1.0 & $1.6 \cdot 10^{-2}$ & 2.1 & $4.9 \cdot 10^{-3}$ & 2.7 & $1.4 \cdot 10^{-4}$ & 3.4 \\
\hline 16 & $4.2 \cdot 10^{-2}$ & 1.1 & $4.3 \cdot 10^{-3}$ & 1.9 & $6.3 \cdot 10^{-4}$ & 3.0 & $1.0 \cdot 10^{-5}$ & 3.8 \\
\hline 32 & $2.0 \cdot 10^{-2}$ & 1.0 & $1.1 \cdot 10^{-3}$ & 2.0 & $8.7 \cdot 10^{-5}$ & 2.9 & - & - \\
\hline 64 & $1.0 \cdot 10^{-2}$ & 1.0 & $2.6 \cdot 10^{-4}$ & 2.0 & - & - & - & - \\
\hline
\end{tabular}

with FEM we observe $\left\|\boldsymbol{u}^{e}-\boldsymbol{u}^{h}\right\|_{1, \Omega} \leqslant \mathcal{O}\left(h^{s}\right)$, with $s=1 / 2$ for crack problems, independent of $p$. Figure 16 showcases the contrast between suboptimal rates for the $H^{1}$-norm for FEM in comparison with MFEM for the interface crack problem, and rates and errors are reported in Table V.

\section{ANALYSIS OF THE METHOD}

In this section, we prove the optimal convergence of the proposed method as the main result. More precisely, we provide an analysis of the method for the problem of finding $\boldsymbol{u}^{h}:=\hat{\boldsymbol{u}}^{h} \circ \boldsymbol{\gamma}^{-1}$, where $\hat{\boldsymbol{u}}^{h} \in \hat{\mathcal{V}}^{h, p}$ is such that (13) holds, with the map $\gamma$ introduced in (6) where $k \geqslant p+1$. We are mostly interested in the error over the physical domain, namely, in an estimate of $\left\|\boldsymbol{u}-\boldsymbol{u}^{h}\right\|_{s, \Omega}, s=0,1$. The proof of this last result is built on the convergence rates in the parametric domain and a norm equivalence between functions in the parametric and physical domains. Crucial to this approach is a proof of the enhanced regularity of the mapped solution $\hat{\boldsymbol{u}}$.

For clarity, we assume that $\Omega$ is open and has the cone property (to apply Sobolev's embedding theorem) and that an exact discretization of the domain $\hat{\Omega}$ is adopted, instead of, for example, an isoparametric approximation. Furthermore, we assume that the only singularity comes from the only crack tip of a Lipschitz-continuous edge crack (i.e., the image of a Lipschitz function $\mathbb{R} \rightarrow \mathbb{R}^{2}$ ) that does not cross itself and thus we consider a mapping of the form (6). The analysis for a re-entrant corner can be accounted for in a similar and simpler manner.

To simplify the statement of the regularity assumption, we let polar coordinates $(r, \theta)$ be continuous all over $\Omega$, i.e., within $\bar{\Omega}$ the value of $\theta$ is discontinuous only across $\mathscr{C}$. We then let $\Theta$ be the minimum real number such that $\theta \in(-\Theta, \Theta)$ for all points of $\Omega$. If $\mathscr{C}$ is straight, then $\Theta=\pi$.

Crack geometries inside $B_{\rho}\left(\boldsymbol{x}_{\top}\right)$ could be quite complex. For the purposes of the subsequent proof, it is useful to keep in mind the image of $B_{\rho}\left(\boldsymbol{x}_{\top}\right)$ with a straight crack emanating from its center. In general, however, a rather mild hypothesis that we shall make is to assume that there exists an extension of the crack that cuts $B_{\rho}\left(\boldsymbol{x}_{\mathrm{T}}\right)$ into two disjoint sets, $B_{+}$and $B_{-}$, such that $\left|\partial B_{ \pm} \cap \partial B_{\rho}\left(\boldsymbol{x}_{\top}\right)\right|>$ 0 , and that both sets satisfy the strong local Lipschitz condition; see Figure 17. This assumption will allow us to apply Poincaré's inequality, a trace inequality, and the divergence theorem on each set 


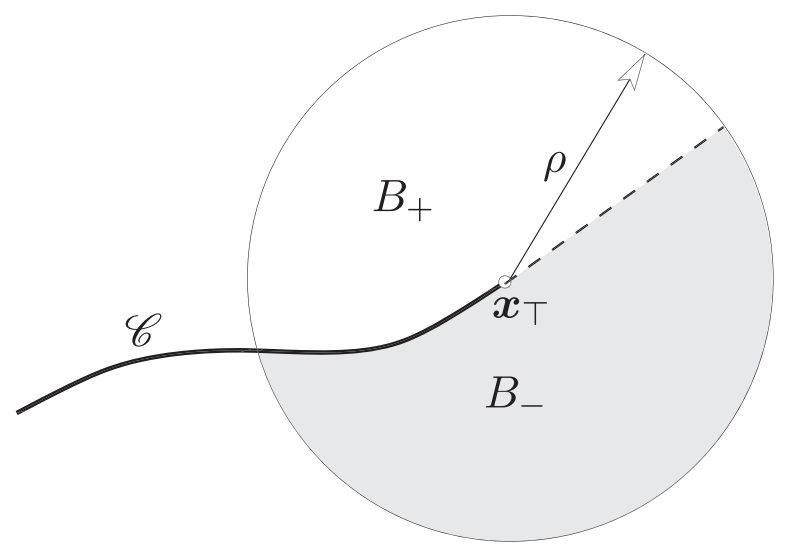

Figure 17. Schematic showing the extension of the crack (dashed line) that cuts $B_{\rho}\left(\boldsymbol{x}_{\top}\right)$ into two disjoint parts, $B_{+}$and $B_{-}$, such that $\left|\partial B_{ \pm} \cap \partial B_{\rho}\left(\boldsymbol{x}_{\top}\right)\right|>0$ and that both sets have the strong Lipschitz property.

(cf. [40]). If $\mathscr{C} \cap B_{\rho}\left(\boldsymbol{x}_{\mathrm{T}}\right)$ is straight, then this extension can be set as extending along the line that contains $\mathscr{C} \cap B_{\rho}\left(\boldsymbol{x}_{\top}\right)$.

We will adopt the following notation to indicate partial derivatives. Let multi-index $\boldsymbol{\alpha}=\left(\alpha_{1}, \alpha_{2}\right) \in$ $\left(\mathbb{N}_{0}\right)^{2}$ and $|\boldsymbol{\alpha}|:=\alpha_{1}+\alpha_{2}$. We then define the operator $D_{x}^{\alpha}:=\left(\partial / \partial x_{1}\right)^{\alpha_{1}}\left(\partial / \partial x_{2}\right)^{\alpha_{2}}$. Likewise, $D_{\hat{x}}^{\alpha}:=$ $\left(\partial / \partial \hat{x}_{1}\right)^{\alpha_{1}}\left(\partial / \partial \hat{x}_{2}\right)^{\alpha_{2}}$, and $D_{(\hat{r}, \hat{\theta})}^{\alpha}:=(\partial / \partial \hat{r})^{\alpha_{1}}(\partial / \partial \hat{\theta})^{\alpha_{2}}$. To avoid cluttering of symbols, we set $\mathscr{D}^{\alpha}:=$ $D_{(\hat{r}, \hat{\theta})}^{\alpha}$.

In the sequel, we first lay out the regularity assumption of the solution and then proceed to the proof. Note that within $\S$ Section 5 the symbol $C$ denotes a generic positive constant independent of the solution $\boldsymbol{u}$ and the mesh size $h$. The value of $C$ may differ at different occurrences.

Assumption 5.1 (Regularity of the solution)

We assume that there exists $k \in \mathbb{N}, k \geqslant 2$, such that $\boldsymbol{b} \in H^{k-2}(\Omega), \boldsymbol{g} \in H^{k-1 / 2}\left(\partial_{d} \Omega\right), \boldsymbol{t} \in H^{k-3 / 2}\left(\partial_{\tau} \Omega\right)$, and that the solution to (2) can be written as

$$
\boldsymbol{u}=\sum_{i=1}^{k-1} r^{\lambda_{i}} \boldsymbol{\psi}_{i}(\theta)+\boldsymbol{u}_{R}
$$

where $\lambda_{i}=i-1 / 2, \boldsymbol{\psi}_{i} \in C^{\infty}[-\Theta, \Theta], \boldsymbol{u}_{R} \in H^{k}\left(\Omega, \mathbb{R}^{2}\right)$. Moreover, there exists $C$ independent of $\boldsymbol{b}$, $\boldsymbol{g}$, and $\boldsymbol{t}$ such that

$$
\sum_{i=1}^{k-1}\left\|\boldsymbol{\psi}_{i}\right\|_{k,(-\Theta, \Theta)}^{2}+\left\|\boldsymbol{u}_{R}\right\|_{k, \Omega}^{2} \leqslant C\left(\|\boldsymbol{b}\|_{k-2, \Omega}^{2}+\|\boldsymbol{g}\|_{k-1 / 2, \partial_{d} \Omega}^{2}+\|\boldsymbol{t}\|_{k-3 / 2, \partial_{\tau} \Omega}^{2}\right) .
$$

We remark that the so-called stress intensity factors are incorporated in the function $\boldsymbol{\psi}_{1}(\theta)$.

Under Assumption 5.1, our main result is summarized in Theorems 5.1 and 5.2.

Theorem 5.1 (Regularity of the mapped solution)

Let $\boldsymbol{u} \in \mathcal{V}$ be the solution to (2) with $k \in \mathbb{N}, k \geqslant 2$ given by Assumption 5.1. Let $\gamma$ be as defined in (6) with $q \in \Pi_{k, m}$ for some $m \geqslant k$. Then $\hat{\boldsymbol{u}}:=\boldsymbol{u} \circ \boldsymbol{\gamma} \in H^{k}\left(\hat{\Omega}, \mathbb{R}^{2}\right)$; moreover, there exists $C>0$ independent of $\boldsymbol{b}, \boldsymbol{g}$, and $\boldsymbol{t}$ such that

$$
\|\hat{\boldsymbol{u}}\|_{k, \hat{\Omega}} \leqslant C\left(\|\boldsymbol{b}\|_{k-2, \Omega}+\|\boldsymbol{g}\|_{k-1 / 2, \partial_{d} \Omega}+\|\boldsymbol{t}\|_{k-3 / 2, \partial_{\tau} \Omega}\right) .
$$

Theorem 5.2 (Optimality of convergence)

Let the assumptions of Theorem 5.1 hold. Let $p \in \mathbb{N}$ with $p \leqslant k-1$, and $\left\{\mathcal{T}^{h}\right\}$ be a quasi-uniform family of subdivisions of $\hat{\Omega}=\gamma^{-1}(\Omega)$ over which finite element spaces $\hat{\mathcal{V}}^{h, p}$ and $\hat{\mathcal{W}}^{h, p}$ are constructed 
following (12). Let $\hat{\boldsymbol{u}}^{h} \in \hat{\mathcal{V}}^{h, p}$ be such that (13) holds, and set $\boldsymbol{u}^{h}:=\hat{\boldsymbol{u}}^{h} \circ \gamma^{-1}$. Then there exists a positive constant $C$ independent of $\boldsymbol{u}$ and $h$ such that

$$
\left|\boldsymbol{u}-\boldsymbol{u}^{h}\right|_{s, \Omega} \leqslant C h^{p+1-s}|\boldsymbol{u} \circ \gamma|_{p+1, \hat{\Omega}}, \quad s=0,1 .
$$

\section{Corollary 5.3}

Let the assumptions of Theorem 5.2 hold. Then there exists a positive constant $C$ independent of $\boldsymbol{u}$ and $h$ such that

$$
\left|\boldsymbol{u}-\boldsymbol{u}^{h}\right|_{s, \Omega} \leqslant C h^{p+1-s}\left(\|\boldsymbol{b}\|_{p-1, \Omega}+\|\boldsymbol{g}\|_{p+1 / 2, \partial_{d} \Omega}+\|\boldsymbol{t}\|_{p-1 / 2, \partial_{\tau} \Omega}\right), \quad s=0,1 .
$$

To prove these main results, we first prove a few lemmas.

Lemma 5.4 (Bivariate chain rule of an arbitrary order)

Let multi-index $\boldsymbol{\alpha}=\left(\alpha_{1}, \alpha_{2}\right) \in\left(\mathbb{N}_{0}\right)^{2}$ and functions $f \in C^{|\boldsymbol{\alpha}|}\left(\mathbb{R}^{2}\right), \boldsymbol{g}=\left(g_{1}, g_{2}\right)$, with $g_{1}, g_{2} \in C^{\boldsymbol{\alpha}}\left(\mathbb{R}^{2}\right)$. Construct the composition of functions

$$
h(\boldsymbol{x})=f\left[g_{1}(\boldsymbol{x}), g_{2}(\boldsymbol{x})\right], \quad \boldsymbol{x}=\left(x_{1}, x_{2}\right) .
$$

Then the partial derivative $D_{x}^{\alpha} h$ is given by

$$
D_{x}^{\alpha} h=\sum_{1 \leqslant|\boldsymbol{\beta}| \leqslant|\boldsymbol{\alpha}|} D_{g}^{\beta} f\left[g_{1}(\boldsymbol{x}), g_{2}(\boldsymbol{x})\right] \sum_{s=1}^{|\alpha|} \sum_{p_{s}(\boldsymbol{\alpha}, \boldsymbol{\beta})}\left(\alpha_{1} ! \alpha_{2} !\right) \prod_{j=1}^{s} \frac{\left(D_{x}^{l_{j}} g_{1}(\boldsymbol{x})\right)^{k_{j}^{(1)}}\left(D_{x}^{l_{j}} g_{2}(\boldsymbol{x})\right)^{k_{j}^{(2)}}}{\left[k_{j}^{(1)} ! k_{j}^{(2)} !\right]\left[l_{j}^{(1)} ! l_{j}^{(2)} !\right]^{\left|\boldsymbol{k}_{j}\right|}},
$$

where $D_{\boldsymbol{g}}^{\beta} f=\left(\partial / \partial g_{1}\right)^{\beta_{1}}\left(\partial / \partial g_{2}\right)^{\beta_{2}} f$, and for $j=1, \ldots, s, \boldsymbol{k}_{j}=\left(k_{j}^{(1)}, k_{j}^{(2)}\right), \boldsymbol{l}_{j}=\left(l_{j}^{(1)}, l_{j}^{(2)}\right)$. Finally,

$$
\begin{gathered}
p_{s}(\boldsymbol{\alpha}, \boldsymbol{\beta})=\left\{\left(\boldsymbol{k}_{1}, \ldots, \boldsymbol{k}_{s} ; \boldsymbol{l}_{1}, \ldots, \boldsymbol{l}_{s}\right)|| \boldsymbol{k}_{i}|>0,| \boldsymbol{l}_{i} \mid>0, i=1, \ldots, s ;\right. \\
\left.\boldsymbol{l}_{1}, \ldots, \boldsymbol{l}_{s} \text { are distinct; } \sum_{i=1}^{s} \boldsymbol{k}_{i}=\boldsymbol{\beta} ; \sum_{i=1}^{s}\left|\boldsymbol{k}_{i}\right| \boldsymbol{l}_{i}=\boldsymbol{\alpha}\right\} .
\end{gathered}
$$

Proof

This is a special case of the main result of [41].

Example applications of Lemma 5.4 for some $\boldsymbol{\alpha}$ 's are given as follows, where the standard partial derivative formulas are recovered:

- $\boldsymbol{\alpha}=(1,0)$, then the only contributions are from $\boldsymbol{\beta}=(0,1)$ or $(1,0)$. We note that

$$
\begin{aligned}
& p_{1}((1,0),(0,1))=\left\{\left(\boldsymbol{k}_{1}=(0,1) ; \boldsymbol{l}_{1}=(1,0)\right)\right\}, \\
& p_{1}((1,0),(1,0))=\left\{\left(\boldsymbol{k}_{1}=(1,0) ; \boldsymbol{l}_{1}=(1,0)\right)\right\} .
\end{aligned}
$$

As a result,

$$
\frac{\partial h}{\partial x_{1}}=\frac{\partial f}{\partial g_{2}}\left[g_{1}(\boldsymbol{x}), g_{2}(\boldsymbol{x})\right] \frac{\partial g_{2}}{\partial x_{1}}+\frac{\partial f}{\partial g_{1}}\left[g_{1}(\boldsymbol{x}), g_{2}(\boldsymbol{x})\right] \frac{\partial g_{1}}{\partial x_{1}} .
$$

- $\boldsymbol{\alpha}=(1,1)$, then the only contributions are from $\boldsymbol{\beta}=(0,1),(1,0),(0,2),(1,1)$, or $(2,0)$. We next find out the $p_{s}$ 's:

$$
\begin{aligned}
p_{1}((1,1),(0,1)) & =\left\{\left(\boldsymbol{k}_{1}=(0,1) ; \boldsymbol{l}_{1}=(1,1)\right)\right\}, \\
p_{1}((1,1),(1,0)) & =\left\{\left(\boldsymbol{k}_{1}=(1,0) ; \boldsymbol{l}_{1}=(1,1)\right)\right\}, \\
p_{2}((1,1),(0,2)) & =\left\{\left(\boldsymbol{k}_{1}=\boldsymbol{k}_{2}=(0,1) ; \boldsymbol{l}_{1}=(0,1), \boldsymbol{l}_{2}=(1,0)\right)\right\}, \\
p_{2}((1,1),(1,1)) & =\left\{\left(\boldsymbol{k}_{1}=(0,1), \boldsymbol{k}_{2}=(1,0) ; \boldsymbol{l}_{1}=(0,1), \boldsymbol{l}_{2}=(1,0)\right),\right. \\
\left(\boldsymbol{k}_{1}\right. & \left.\left.=(1,0), \boldsymbol{k}_{2}=(0,1) ; \boldsymbol{l}_{1}=(0,1), \boldsymbol{l}_{2}=(1,0)\right)\right\}, \\
p_{2}((1,1),(2,0)) & =\left\{\left(\boldsymbol{k}_{1}=\boldsymbol{k}_{2}=(1,0) ; \boldsymbol{l}_{1}=(0,1), \boldsymbol{l}_{2}=(1,0)\right)\right\} .
\end{aligned}
$$


From this, we obtain

$$
\begin{aligned}
\frac{\partial^{2} h}{\partial x_{1} \partial x_{2}}= & \frac{\partial f}{\partial g_{2}}\left[g_{1}(\boldsymbol{x}), g_{2}(\boldsymbol{x})\right] \frac{\partial^{2} g_{2}}{\partial x_{1} \partial x_{2}}+\frac{\partial f}{\partial g_{1}}\left[g_{1}(\boldsymbol{x}), g_{2}(\boldsymbol{x})\right] \frac{\partial^{2} g_{1}}{\partial x_{1} \partial x_{2}}+\frac{\partial^{2} f}{\partial g_{2}^{2}}\left[g_{1}(\boldsymbol{x}), g_{2}(\boldsymbol{x})\right] \frac{\partial g_{2}}{\partial x_{2}} \frac{\partial g_{2}}{\partial x_{1}} \\
& +\frac{\partial^{2} f}{\partial g_{1} \partial g_{2}}\left[g_{1}(\boldsymbol{x}), g_{2}(\boldsymbol{x})\right]\left(\frac{\partial g_{2}}{\partial x_{2}} \frac{\partial g_{1}}{\partial x_{1}}+\frac{\partial g_{1}}{\partial x_{2}} \frac{\partial g_{2}}{\partial x_{1}}\right)+\frac{\partial^{2} f}{\partial g_{1}^{2}}\left[g_{1}(\boldsymbol{x}), g_{2}(\boldsymbol{x})\right] \frac{\partial g_{1}}{\partial x_{2}} \frac{\partial g_{1}}{\partial x_{1}} .
\end{aligned}
$$

Lemma 5.5 (Basic properties of the map $\gamma$ )

Let $\boldsymbol{\gamma}$ be given by (6) with $q \in \Pi_{k, m}$ and $j=\operatorname{det}(\nabla \gamma)$. Let $\hat{\boldsymbol{x}} \in B_{\rho}\left(\boldsymbol{x}_{\top}\right)$ and $\hat{\boldsymbol{r}}=\left\|\hat{\boldsymbol{x}}-\boldsymbol{x}_{\top}\right\|, r=$ $\left\|\boldsymbol{\gamma}(\hat{\boldsymbol{x}})-\boldsymbol{x}_{\mathrm{T}}\right\|$. Then $\boldsymbol{\gamma} \in C^{k}\left(\mathbb{R}^{2}, \mathbb{R}^{2}\right)$, and there exist $C_{1}, C_{2}, C_{3}>0$ independent of $\hat{\boldsymbol{x}}$ such that for all $\hat{r} \in(0, \rho]$,

$$
\begin{gathered}
\left|\frac{d^{s} r}{d \hat{r}^{j}}\right| \leqslant C_{2} \hat{r}^{2 m-s}, \quad s \in \mathbb{N}, 0 \leqslant s \leqslant k, \\
\left\|D_{\hat{x}}^{\alpha} \gamma\right\| \leqslant C_{2} \hat{r}^{2 m-|\alpha|} \leqslant C_{3} r^{1-|\alpha| /(2 m)}, \quad \alpha \in\left(\mathbb{N}_{0}\right)^{2}, 0 \leqslant|\alpha| \leqslant k \\
C_{1} r^{-1+1 /(2 m)} \leqslant\left\|(\nabla \gamma)^{-1}\right\| \leqslant C_{2} r^{-1+1 /(2 m)}, \\
C_{1} r^{-2+1 / m} \leqslant j^{-1} \leqslant C_{2} r^{-2+1 / m} .
\end{gathered}
$$

Proof

These inequalities directly follow from (10) and (8). The fact that $\gamma \in C^{k}\left(\mathbb{R}^{2}, \mathbb{R}^{2}\right)$ follows after the behavior of the derivatives of $\boldsymbol{\gamma}$ near $\boldsymbol{x}_{\mathrm{T}}$ is bounded by (17b).

As a special case, taking $\boldsymbol{\alpha}=(0,0)$ in (17b) yields that there exist $C_{1}, C_{2}>0$ independent of $\hat{\boldsymbol{x}}$ such that for all $\hat{r} \in(0, \rho]$,

$$
C_{1} \hat{r}^{2 m} \leqslant r \leqslant C_{2} \hat{r}^{2 m}
$$

\section{Lemma 5.6}

Let the assumptions of Theorem 5.1 hold. Let $v \in H^{k}(\Omega)$ and $\hat{v}=v \circ \gamma$. Then $\hat{v} \in H^{k}(\hat{\Omega})$ and there exists $C$ independent of $v$ such that

Proof

$$
\|\hat{v}\|_{k, \hat{\Omega}} \leqslant C\|v\|_{k, \Omega}
$$

We first prove that

$$
\|\hat{v}\|_{0, \hat{\Omega}} \leqslant C\|v\|_{2, \Omega} \text {. }
$$

Because $\Omega$ has the cone property, applying the Sobolev embedding theorem yields that $v \in C_{B}^{0}(\Omega)$, and that $\|v\|_{0, \infty, \Omega} \leqslant C\|v\|_{2, \Omega}$. As a result, $\hat{v} \in C_{B}^{0}(\Omega)$ and $\|\hat{v}\|_{0, \hat{\Omega}} \leqslant C\|\hat{v}\|_{0, \infty, \hat{\Omega}}=C\|v\|_{0, \infty, \Omega} \leqslant$ $C\|v\|_{2, \Omega}$, where we have used the bijectivity and continuity of $\gamma$.

We next define, for each $\boldsymbol{\alpha}$ such that $1 \leqslant|\boldsymbol{\alpha}| \leqslant k$, a provisional expression for $D_{\hat{x}}^{\alpha} \hat{v}$ given by

$$
\tilde{D}_{\hat{\boldsymbol{x}}}^{\alpha} \hat{v}=\sum_{1 \leqslant|\beta| \leqslant|\boldsymbol{\alpha}|} D_{\boldsymbol{x}}^{\beta} v\left[\gamma_{1}(\hat{\boldsymbol{x}}), \gamma_{2}(\hat{\boldsymbol{x}})\right] \sum_{s=1}^{|\boldsymbol{\alpha}|} \sum_{p_{s}(\boldsymbol{\alpha}, \boldsymbol{\beta})}\left(\alpha_{1} ! \alpha_{2} !\right) \prod_{j=1}^{s} \frac{\left(D_{\hat{\boldsymbol{x}}}^{l_{j}} \gamma_{1}(\hat{\boldsymbol{x}})\right)^{k_{j}^{(1)}}\left(D_{\hat{\boldsymbol{x}}}^{\boldsymbol{l}_{j}} \gamma_{2}(\hat{\boldsymbol{x}})\right)^{k_{j}^{(2)}}}{\left[k_{j}^{(1)} ! k_{j}^{(2)} !\right]\left[l_{j}^{(1)} ! l_{j}^{(2)} !\right]^{\left|\boldsymbol{k}_{j}\right|}} .
$$

We now show that for all $\boldsymbol{\alpha}$ such that $1 \leqslant|\alpha| \leqslant k$,

$$
\left\|\tilde{D}_{\hat{x}}^{\alpha} \hat{v}\right\|_{0, \hat{\Omega}} \leqslant C\|v\|_{k, \Omega}
$$

and then that $\tilde{D}_{\hat{x}}^{\alpha} \hat{v}$ is in fact the $\alpha$-weak derivative of $\hat{v}$. 
To this end, we first note the finiteness finite number of terms in $p_{s}(\boldsymbol{\alpha}, \boldsymbol{\beta})$ and that $\boldsymbol{\gamma}$ is the identity in $\hat{\Omega} \backslash B_{\rho}\left(\boldsymbol{x}_{\top}\right)$; hence, we just need to prove that for each member of $p_{s}(\boldsymbol{\alpha}, \boldsymbol{\beta})$,

$$
\int_{B_{\rho}\left(\boldsymbol{x}_{\top}\right) \cap \Omega}\left(D_{x}^{\beta} v\right)^{2}\left[\prod_{j=1}^{s}\left(D_{\hat{x}}^{l_{j}} \gamma_{1}\right)^{2 k_{j}^{(1)}}\left(D_{\hat{x}}^{l_{j}} \gamma_{2}\right)^{2 k_{j}^{(2)}}\right] \circ \gamma^{-1} j^{-1} d \Omega \leqslant C\|v\|_{k, B_{\rho}\left(\boldsymbol{x}_{\top}\right) \cap \Omega}^{2} .
$$

To proceed, we apply (16), (17b), and (17d) to obtain

$$
\begin{aligned}
& \int_{B_{\rho}\left(x_{\top}\right) \Omega \Omega}\left(D_{x}^{\beta} v\right)^{2}\left[\prod_{j=1}^{s}\left(D_{\hat{x}}^{l_{j}} \gamma_{1}\right)^{2 k_{j}^{(1)}}\left(D_{\hat{x}}^{l_{j}} \gamma_{2}\right)^{2 k_{j}^{(2)}}\right] \circ \gamma^{-1} j^{-1} d \Omega \\
\leqslant & C \int_{B_{\rho}\left(x_{\top}\right) \Omega \Omega} r^{-2+1 / m} r^{2|\beta|-|\alpha| / m}\left\|D_{x}^{\beta} v\right\|^{2} d \Omega \\
\leqslant & C \int_{B_{\rho}\left(x_{\top}\right) \Omega \Omega} r^{2(|\beta|-1)-(|\alpha|-1) / m}\left\|D_{x}^{\beta} v\right\|^{2} d \Omega .
\end{aligned}
$$

If $|\boldsymbol{\beta}| \geqslant 2$, then $2(|\boldsymbol{\beta}|-1)-(|\boldsymbol{\alpha}|-1) / m>0$, and (21) holds; otherwise, for $|\boldsymbol{\beta}|=1$, we invoke the divergence theorem ${ }^{\ddagger}$ on the last term above to write

$$
\begin{aligned}
& \left(2-\frac{|\boldsymbol{\alpha}|-1}{m}\right) \int_{B_{\rho}\left(\boldsymbol{x}_{\top}\right) \cap \Omega} r^{-(|\alpha|-1) / m}\left\|D_{x}^{\beta} v\right\|^{2} d \Omega \\
= & \int_{B_{\rho}\left(\boldsymbol{x}_{\top}\right) \cap \Omega} \operatorname{div}\left[r^{1-(|\boldsymbol{\alpha}|-1) / m} \mathbf{e}_{r}\right]\left\|D_{x}^{\beta} v\right\|^{2} d \Omega \\
= & -\int_{B_{\rho}\left(\boldsymbol{x}_{\top}\right) \cap \Omega} r^{1-(|\boldsymbol{\alpha}|-1) / m} \mathbf{e}_{r} \cdot \nabla\left(\left\|D_{x}^{\beta} v\right\|^{2}\right) d \Omega \\
& +\int_{\partial\left[B_{\rho}\left(\boldsymbol{x}_{\top}\right) \cap \Omega\right]}^{r^{1-(|\alpha|-1) / m} \mathbf{e}_{r} \cdot n\left\|D_{x}^{\beta} v\right\|^{2} d \partial \Omega} \\
\leqslant & C\left|\int_{B_{\rho}\left(\boldsymbol{x}_{\top}\right) \cap \Omega}\left(D_{x}^{\beta} v\right) \cdot \frac{\partial}{\partial r}\left(D_{x}^{\beta} v\right) d \Omega\right|+C \int_{\partial\left[B_{\rho}\left(\boldsymbol{x}_{\top}\right) \cap \Omega\right]}\left\|D_{x}^{\beta} v\right\|^{2} d \Gamma \\
\leqslant & C\|v\|_{2, \Omega}^{2},
\end{aligned}
$$

where we have invoked the Cauchy-Schwarz inequality and the trace inequality in the last step. As a result, (21) is also true for the case of $|\boldsymbol{\beta}|=1$.

It remains to prove that the locally integrable function $\tilde{D}_{\hat{x}}^{\alpha} \hat{v}$ is the weak derivative of $\hat{v}$ with index $\alpha$, which amounts to prove that, for any test function $\phi \in \tilde{C}_{c}^{\infty}(\hat{\Omega})$,

$$
(-1)^{|\alpha|} \int_{\hat{\Omega}} \hat{v} D_{\hat{x}}^{\alpha} \phi d \hat{\Omega}-\int_{\hat{\Omega}} \phi \tilde{D}_{\hat{x}}^{\alpha} \hat{v} d \hat{\Omega}=0 .
$$

To this end, for $\varepsilon>0$, we let $J_{\varepsilon}$ be a mollifier function with $\operatorname{supp}\left(J_{\varepsilon}\right) \subset B_{\varepsilon}(\mathbf{0})$, and $v_{\varepsilon}:=J_{\varepsilon} * v$, where $*$ denotes convolution over $\mathbb{R}^{2}$ :

$$
v_{\varepsilon}(\boldsymbol{x})=\int_{\mathbb{R}^{2}} J_{\varepsilon}(\boldsymbol{x}-\boldsymbol{y}) v(\boldsymbol{y}) d \boldsymbol{\Omega}(\boldsymbol{y}),
$$

with $v$ extended by zero over $\mathbb{R}^{2} \backslash \Omega$. With the same arguments that yield (19), it is clear that $v_{\varepsilon} \circ \gamma \in$ $L^{2}(\hat{\Omega})$. Moreover, from Lemma 5.4,

$$
(-1)^{|\alpha|} \int_{\hat{\Omega}} v_{\varepsilon} \circ \gamma D_{\hat{x}}^{\alpha} \phi d \hat{\Omega}=\int_{\hat{\Omega}} \phi \tilde{D}_{\hat{x}}^{\alpha}\left(v_{\varepsilon} \circ \gamma\right) d \hat{\Omega}
$$

\footnotetext{
The use of the divergence theorem is justified by the strong local Lipschitz condition of $B^{ \pm}$, which implies that $W^{1,1}\left(B^{ \pm}\right)$ is dense in $C^{\infty}\left(\overline{B^{ \pm}}\right)$.
} 
Using (24) on the left-hand side of (23) and applying the Cauchy-Schwarz inequality, (19), and (20) (with $v$ replaced by $v-v_{\varepsilon}$ ) yields

$$
\begin{aligned}
& \left|(-1)^{|\alpha|} \int_{\hat{\Omega}} \hat{v} D_{\hat{x}}^{\alpha} \phi d \hat{\Omega}-\int_{\hat{\Omega}} \phi \tilde{D}_{\hat{x}}^{\alpha} \hat{v} d \hat{\Omega}\right| \\
\leqslant & \left|\int_{\hat{\Omega}}\left(\hat{v}-v_{\varepsilon} \circ \gamma\right) D_{\hat{x}}^{\alpha} \phi d \hat{\Omega}\right|+\left|\int_{\hat{\Omega}} \phi\left[\tilde{D}_{\hat{x}}^{\alpha} \hat{v}-\tilde{D}_{\hat{x}}^{\alpha}\left(v_{\varepsilon} \circ \gamma\right)\right] d \hat{\Omega}\right| \\
\leqslant & \|\phi\|_{k, \hat{\Omega}}\left\|\hat{v}-v_{\varepsilon} \circ \gamma\right\|_{0, \hat{\Omega}}+\|\phi\|_{0, \hat{\Omega}}\left\|\tilde{D}_{\hat{x}}^{\alpha}\left(\hat{v}-v_{\varepsilon} \circ \gamma\right)\right\|_{0, \hat{\Omega}} \\
\leqslant & C\left\|\hat{v}-v_{\varepsilon} \circ \gamma\right\|_{0, \hat{\Omega}}+C\left\|\hat{v}-v_{\varepsilon} \circ \gamma\right\|_{k, \hat{\Omega}} \\
\leqslant & C\left\|v-v_{\varepsilon}\right\|_{k, \Omega} .
\end{aligned}
$$

Passing to the limit $\varepsilon \searrow 0$ and applying [42, Lemma 3.16] yields (23).

Lemma 5.7 (Relation of seminorms)

Let $w \in H^{1}(\Omega)$ and $\hat{w}=w \circ \gamma$ under the assumptions of Theorem 5.1. Then there exists $C$ independent of $w$ such that

$$
C^{-1}|\hat{w}|_{1, \hat{\Omega}} \leqslant|w|_{1, \Omega} \leqslant C|\hat{w}|_{1, \hat{\Omega}}
$$

Proof

The inequality $C^{-1}|\hat{w}|_{1, \hat{\Omega}} \leqslant|w|_{1, \Omega}$ follows from a special case of (22), which was proved when we proved Lemma 5.6.

To prove $|w|_{1, \Omega} \leqslant C|\hat{w}|_{1, \hat{\Omega}}$, we start from Cauchy-Schwarz inequality to obtain

$$
|w|_{1, \Omega}^{2}=\int_{\hat{\Omega}}\left|\nabla \hat{w} \cdot(\nabla \gamma)^{-1}\right|^{2} j d \hat{\Omega} \leqslant \int_{\hat{\Omega}}|\nabla \hat{w}|^{2}\left|(\nabla \gamma)^{-1}\right|^{2} j d \hat{\Omega} .
$$

Hence, it remains to show that there exists $C>0$ such that for all $\hat{\boldsymbol{x}} \in \hat{\Omega},\left|(\nabla \gamma)^{-1}\right|^{2} j \leqslant C$, which follows from (17c) and (17d).

\section{Corollary 5.8}

The operator $a_{\gamma}(\cdot, \cdot)$ is continuous and coercive in $H^{1}\left(\hat{\Omega} ; \mathbb{R}^{2}\right)$.

\section{Proof}

This corollary is a direct consequence of (5) and Lemma 5.7.

We now proceed to prove the main results.

\section{Proof of Theorem 5.1}

For any $\hat{\boldsymbol{x}} \in B_{\rho}\left(\boldsymbol{x}_{\mathrm{T}}\right) \cap \hat{\Omega}$, we let $\hat{r}$ and $r$ be given by the assumptions of Lemma 5.5. Applying the regularity assumption (15) yields

$$
\hat{\boldsymbol{u}}=\boldsymbol{u} \circ \boldsymbol{\gamma}=\sum_{i=1}^{k-1} \hat{\boldsymbol{u}}_{i}+\hat{\boldsymbol{u}}_{R}, \quad \hat{\boldsymbol{u}}_{i}:=r^{\lambda_{i}} \boldsymbol{\Psi}_{i}(\hat{\theta}), \quad \hat{\boldsymbol{u}}_{R}:=\boldsymbol{u}_{R} \circ \gamma,
$$

where, due to $\gamma, \hat{\theta}=\theta$. Applying Lemma 5.6 to $\boldsymbol{u}_{R}$ yields $\left\|\hat{\boldsymbol{u}}_{R}\right\|_{k, \hat{\Omega}} \leqslant C\left\|\boldsymbol{u}_{R}\right\|_{k, \Omega}$, and we conclude that the theorem holds if there exists $C$ independent of $\boldsymbol{u}$ such that

$$
\left\|\hat{\boldsymbol{u}}_{i}\right\|_{k, \hat{\Omega}} \leqslant C\left\|\boldsymbol{\psi}_{i}\right\|_{k,(-\Theta, \Theta)}, \quad i=1, \ldots, k-1 .
$$

To this end, we will prove that there exists $C>0$ such that for any multi-index $\boldsymbol{\alpha}=\left(\alpha_{1}, \alpha_{2}\right) \in\left(\mathbb{N}_{0}\right)^{2}$ with $0 \leqslant|\alpha| \leqslant k$,

$$
\left\|D_{\hat{x}}^{\alpha} \hat{u}_{i}\right\|_{0, \hat{\Omega}} \leqslant C\left\|\boldsymbol{\psi}_{i}\right\|_{|\alpha|,(-\Theta, \Theta)} .
$$


We first prove the case of $|\boldsymbol{\alpha}|=0$, that is, $\alpha=(0,0)$. In this case, from (18),

$$
\left\|\hat{\boldsymbol{u}}_{i}\right\|_{0, \hat{\Omega}}^{2} \leqslant \int_{0}^{\hat{r}_{\max }} r^{2 \lambda_{i} \hat{r}} d \hat{r} \int_{-\Theta}^{\Theta} \boldsymbol{\psi}_{i}^{2} d \hat{\theta} \leqslant C\left\|\boldsymbol{\psi}_{i}\right\|_{0,(-\Theta, \Theta)}^{2},
$$

where $\hat{r}_{\max }:=\sup _{\hat{\boldsymbol{x}} \in \hat{\Omega}}\left\|\hat{\boldsymbol{x}}-\boldsymbol{x}_{\top}\right\|$.

For the case of $1 \leqslant|\boldsymbol{\alpha}| \leqslant k$, we apply Lemma 5.4 to each component of $\hat{\boldsymbol{u}}_{i}[\hat{r}(\hat{\boldsymbol{x}}), \hat{\theta}(\hat{\boldsymbol{x}})]$ to write for any $\hat{\boldsymbol{x}} \neq \mathbf{0}$

$$
D_{\hat{\boldsymbol{x}}}^{\alpha} \hat{\boldsymbol{u}}_{i}=\sum_{1 \leqslant|\boldsymbol{\beta}| \leqslant|\boldsymbol{\alpha}|} \mathscr{D}^{\beta} \hat{\boldsymbol{u}}_{i}[\hat{r}(\hat{\boldsymbol{x}}), \hat{\theta}(\hat{\boldsymbol{x}})] \sum_{s=1}^{|\alpha|} \sum_{p_{s}(\boldsymbol{\alpha}, \boldsymbol{\beta})}\left(\alpha_{1} ! \alpha_{2} !\right) \prod_{j=1}^{s} \frac{\left(D_{\hat{\boldsymbol{x}}}^{\boldsymbol{l}_{j}} \hat{r}(\hat{\boldsymbol{x}})\right)^{k_{j}^{(1)}}\left(D_{\hat{\boldsymbol{x}}}^{\boldsymbol{l}_{j}} \hat{\theta}(\hat{\boldsymbol{x}})\right)^{k_{j}^{(2)}}}{\left[k_{j}^{(1)} ! k_{j}^{(2)} !\right]\left[l_{j}^{(1)} ! l_{j}^{(2)} !\right]^{\left|\boldsymbol{k}_{j}\right|}}
$$

where we have abused notations in regarding $\hat{\boldsymbol{u}}_{i}$ as a function of either $\hat{\boldsymbol{x}}=\left(\hat{x}_{1}, \hat{x}_{2}\right)$ or $(\hat{r}, \hat{\theta})$. Additionally, we have taciltly used the fact that functions $\hat{r}(\hat{\boldsymbol{x}})$ and $\hat{\theta}(\hat{\boldsymbol{x}})$ belong to $C^{\infty}\left(\mathbb{R}^{2} \backslash\{\boldsymbol{0}\}\right)$, needed for Lemma 5.4.

Next, we observe that, for each term in $p_{s}(\boldsymbol{\alpha}, \boldsymbol{\beta})$, by induction we have

$$
\left|D_{\hat{x}}^{l_{j}} \hat{r}\right| \leqslant C \hat{r}^{1-\left|l_{j}\right|}, \quad\left|D_{\hat{x}}^{l_{j}} \hat{\theta}\right| \leqslant C \hat{r}^{-\left|l_{j}\right|}
$$

Moreover, from the Faa di Bruno formula of one-dimension (see, again, [41]) combined with (18), we obtain

$$
\left\|\mathscr{D}^{\beta} \hat{\boldsymbol{u}}_{i}\right\| \leqslant C \hat{r}^{2 m \lambda_{i}-\beta_{1}}\left\|\frac{d^{\beta_{2}} \boldsymbol{\psi}_{i}}{d \hat{\theta}^{\beta_{2}}}\right\| .
$$

As a result,

$$
\left\|D_{\hat{x}}^{\alpha} \hat{\boldsymbol{u}}_{i}\right\|^{2} \leqslant C \sum_{\beta_{1}=0}^{|\alpha|} \hat{r}^{4 m \lambda_{i}-2 \beta_{1}+\sum_{j=1}^{s}\left[2 k_{j}^{(1)}\left(1-\left|l_{j}\right|\right)+2 k_{j}^{(2)}\left(-\left|l_{j}\right|\right)\right]} \sum_{\beta_{2}=0}^{|\alpha|}\left\|\frac{d^{\beta_{2}} \boldsymbol{\Psi}_{i}}{d \hat{\theta}^{\beta_{2}}}\right\|^{2} .
$$

Note that from (16),

$$
-2 \beta_{1}+\sum_{j=1}^{s}\left[2 k_{j}^{(1)}\left(1-\left|\boldsymbol{l}_{j}\right|\right)+2 k_{j}^{(2)}\left(-\left|\boldsymbol{l}_{j}\right|\right)\right]=\underbrace{-2 \beta_{1}+2 \sum_{j=1}^{s} k_{j}^{(1)}}_{=0}-2 \sum_{j=1}^{s}\left|\boldsymbol{k}_{j}\right|\left|\boldsymbol{l}_{j}\right|=-2|\boldsymbol{\alpha}| .
$$

Thus,

$$
\left\|D_{\hat{x}}^{\alpha} \hat{u}_{i}\right\|^{2} \leqslant C \hat{r}^{4 m \lambda_{i}-2|\alpha|} \sum_{\beta_{2}=0}^{|\alpha|}\left\|\frac{d^{\beta_{2}} \boldsymbol{\Psi}_{i}}{d \hat{\theta}^{\beta_{2}}}\right\|^{2} .
$$

Finally, because $\lambda_{i} \geqslant 1 / 2$ and $m \geqslant k \geqslant|\alpha|$, the theorem holds.

Proof of Theorem 5.2

The case of $s=1$ is straightforward. We first write

$$
\left|\boldsymbol{u}-\boldsymbol{u}^{h}\right|_{1, \Omega} \leqslant\left|\boldsymbol{u}-\boldsymbol{u}_{I}\right|_{1, \Omega}+\left|\boldsymbol{u}_{I}-\boldsymbol{u}^{h}\right|_{1, \Omega}
$$

where $\boldsymbol{u}_{I}:=\hat{\mathcal{I}}^{h} \hat{\boldsymbol{u}} \circ \gamma^{-1}$ and $\hat{\mathcal{I}}^{h}$ is the classical interpolation operator such that $\hat{\mathcal{I}}^{h} \hat{\boldsymbol{u}} \in \hat{\mathcal{V}}^{h, p}$ and that $\hat{\mathcal{I}}^{h} \hat{\boldsymbol{u}}$ and $\hat{\boldsymbol{u}}$ coincide at all nodes of $\mathcal{T}^{h}$. By construction, $\boldsymbol{u}_{I}-\boldsymbol{u}^{h}=0$ on $\partial_{d} \Omega$, and thus, $\boldsymbol{u}_{I}-\boldsymbol{u}^{h} \in$ $\hat{\mathcal{W}}^{h, p} \circ \gamma^{-1}$.

From the coercivity of $a(\cdot, \cdot)$ and the Galerkin orthogonality of $\boldsymbol{u}^{h}$,

$$
\begin{aligned}
& \quad\left|\boldsymbol{u}_{I}-\boldsymbol{u}^{h}\right|_{1, \Omega}^{2} \leqslant C a\left(\boldsymbol{u}_{I}-\boldsymbol{u}^{h}, \boldsymbol{u}_{I}-\boldsymbol{u}^{h}\right)=C a\left(\boldsymbol{u}_{I}-\boldsymbol{u}, \boldsymbol{u}_{I}-\boldsymbol{u}^{h}\right)+\underbrace{C a\left(\boldsymbol{u}-\boldsymbol{u}^{h}, \boldsymbol{u}_{I}-\boldsymbol{u}^{h}\right)}_{=0} \\
& \leqslant C\left|\boldsymbol{u}-\boldsymbol{u}_{I}\right|_{1, \Omega}\left|\boldsymbol{u}_{I}-\boldsymbol{u}^{h}\right|_{1, \Omega},
\end{aligned}
$$


or $\left|\boldsymbol{u}_{I}-\boldsymbol{u}^{h}\right|_{1, \Omega} \leqslant C\left|\boldsymbol{u}-\boldsymbol{u}_{I}\right|_{1, \Omega}$. As a result, with Lemma 5.7 and a standard interpolation error estimate, (25) is simplified to

$$
\left|\boldsymbol{u}-\boldsymbol{u}^{h}\right|_{1, \Omega} \leqslant C\left|\boldsymbol{u}-\boldsymbol{u}_{I}\right|_{1, \Omega} \leqslant C\left|\hat{\boldsymbol{u}}-\hat{\mathcal{I}}^{h} \hat{\boldsymbol{u}}\right|_{1, \hat{\Omega}} \leqslant C h^{p}|\hat{\boldsymbol{u}}|_{p+1, \hat{\Omega}}
$$

For $s=0$, we first note that a standard interpolation error estimate yields

$$
\left\|\boldsymbol{u}-\boldsymbol{u}_{I}\right\|_{0, \Omega} \leqslant C h^{p+1}|\hat{\boldsymbol{u}}|_{p+1, \hat{\Omega}}
$$

Hence, it is sufficient to prove

$$
\left\|\boldsymbol{u}_{I}-\boldsymbol{u}^{h}\right\|_{0, \Omega} \leqslant C h^{p+1}|\hat{\boldsymbol{u}}|_{p+1, \hat{\Omega}}
$$

To this end, we employ the standard argument by considering the solution $\boldsymbol{w} \in \mathcal{W}$ of the problem

$$
a(\boldsymbol{v}, \boldsymbol{w})=\left(\boldsymbol{u}_{I}-\boldsymbol{u}^{h}, \boldsymbol{v}\right), \quad \forall \boldsymbol{v} \in \mathcal{W},
$$

and its MFEM approximation $\boldsymbol{w}^{h} \in \hat{\mathcal{W}}^{h, p} \circ \gamma^{-1}$ such that

$$
a\left(\boldsymbol{v}, \boldsymbol{w}^{h}\right)=\left(\boldsymbol{u}_{I}-\boldsymbol{u}^{h}, \boldsymbol{v}\right), \quad \forall \boldsymbol{v} \in \hat{\mathcal{W}}^{h, p} \circ \gamma^{-1} .
$$

Then applying (26) and Theorem 5.1 to $\hat{w}=\boldsymbol{w} \circ \boldsymbol{\gamma}$ with $k=2$ yields

$$
\left|\boldsymbol{w}-\boldsymbol{w}^{h}\right|_{1, \Omega} \leqslant C h|\hat{\boldsymbol{w}}|_{2, \hat{\Omega}} \leqslant C h\left\|\boldsymbol{u}_{I}-\boldsymbol{u}^{h}\right\|_{0, \Omega} .
$$

To proceed, we note that $\boldsymbol{u}_{I}-\boldsymbol{u}^{h} \in \mathcal{W}$, and hence from (28), the Galerkin orthogonality of $\boldsymbol{w}^{h}$, the continuity of $a(\cdot, \cdot)$, a standard interpolation error estimate, and (26),

$$
\begin{aligned}
\left\|\boldsymbol{u}_{I}-\boldsymbol{u}^{h}\right\|_{0, \Omega}^{2} & =a\left(\boldsymbol{u}_{I}-\boldsymbol{u}^{h}, \boldsymbol{w}\right)=a\left(\boldsymbol{u}_{I}-\boldsymbol{u}^{h}, \boldsymbol{w}-\boldsymbol{w}^{h}\right) \leqslant C\left|\boldsymbol{u}_{I}-\boldsymbol{u}^{h}\right|_{1, \Omega}\left|\boldsymbol{w}-\boldsymbol{w}^{h}\right|_{1, \Omega} \\
& \leqslant C\left(\left|\boldsymbol{u}-\boldsymbol{u}_{I}\right|_{1, \Omega}+\left|\boldsymbol{u}-\boldsymbol{u}^{h}\right|_{1, \Omega}\right) h\left\|\boldsymbol{u}_{I}-\boldsymbol{u}^{h}\right\|_{0, \Omega} \\
& \leqslant C h^{p+1}|\hat{\boldsymbol{u}}|_{p+1, \hat{\Omega}}\left\|\boldsymbol{u}_{I}-\boldsymbol{u}^{h}\right\|_{0, \Omega}
\end{aligned}
$$

and thus (27) holds.

\section{APPENDIX A: GRADIENT OF THE MAPPING}

For convenience, here we provide the expression of the gradient of the mapping, $\nabla \gamma$, and that of its inverse. The gradient of $\gamma$ is given by

$$
\nabla \boldsymbol{\gamma}=\mathbf{1}-\sum_{\tau=1}^{t}\left[\left(1-q\left(\eta_{\tau}\right) / \eta_{\tau}\right) \mathbf{1}+\left(q\left(\eta_{\tau}\right) / \eta_{\tau}-q^{\prime}\left(\eta_{\tau}\right)\right) \mathbf{e}_{\hat{r}, \tau} \otimes \mathbf{e}_{\hat{r}, \tau}\right],
$$

where $\mathbf{e}_{\hat{r}, \tau}:=\left(\hat{\boldsymbol{x}}-\boldsymbol{x}_{\mathrm{T}, \tau}\right) / \hat{r}_{\tau}$ is the unit vector in the direction of $\hat{\boldsymbol{x}}-\boldsymbol{x}_{\mathrm{T}, \tau}$. Because the mapping is local to each singularity, within $B_{\rho_{\tau}}\left(\boldsymbol{x}_{\mathrm{T}, \tau}\right)$, we have

$$
\begin{aligned}
\nabla \boldsymbol{\gamma} & =\mathbf{1}-\left[\left(1-q\left(\eta_{\tau}\right) / \eta_{\tau}\right) \mathbf{1}+\left(q\left(\eta_{\tau}\right) / \eta_{\tau}-q^{\prime}\left(\eta_{\tau}\right)\right) \mathbf{e}_{\hat{r}, \tau} \otimes \mathbf{e}_{\hat{r}, \tau}\right], \\
& =q^{\prime}\left(\eta_{\tau}\right) \mathbf{e}_{\hat{r}, \tau} \otimes \mathbf{e}_{\hat{r}, \tau}+q\left(\eta_{\tau}\right) / \eta_{\tau} \mathbf{e}_{\hat{\theta}, \tau} \otimes \mathbf{e}_{\hat{\theta}, \tau},
\end{aligned}
$$

from which it follows that

$$
(\nabla \gamma)^{-1}= \begin{cases}\frac{1}{q^{\prime}\left(\eta_{\tau}\right)} \mathbf{e}_{\hat{r}, \tau} \otimes \mathbf{e}_{\hat{r}, \tau}+\frac{\eta_{\tau}}{q\left(\eta_{\tau}\right)} \mathbf{e}_{\hat{\theta}, \tau} \otimes \mathbf{e}_{\hat{\theta}, \tau}, & \text { in } B_{\rho_{\tau}}\left(\boldsymbol{x}_{\mathrm{T}, \tau}\right), \\ \mathbf{1}, & \text { otherwise, }\end{cases}
$$

where $\mathbf{e}_{\hat{\theta}, \tau}$ is the basis vector of the $\hat{\theta}$-coordinate of the $\tau$ th singularity. 


\section{APPENDIX B: REMARKS ON THE IMPLEMENTATION}

To expand on the presentation of Section 3.2, we let $\left\{N_{a}\right\}_{a}$ denote the shape functions constructed on the subdivision $\mathcal{T}^{h}$ of $\hat{\Omega}^{h}$ such that

$$
\hat{\boldsymbol{u}}^{h}=N_{a} \boldsymbol{u}_{a}, \quad \hat{\boldsymbol{w}}^{h}=N_{a} \boldsymbol{w}_{a},
$$

where repeated indices imply a summation over them. Here, $\boldsymbol{u}_{a}$ and $\boldsymbol{w}_{a}$ are the nodal values of the displacement field and test functions over spaces of Lagrange finite elements. We remark that the shape functions are constructed over the parametric domain; on the physical domain, they will be given by $n_{a}=N_{a} \circ \gamma^{-1}$. The above implies that, if polynomial shape functions $N_{a}$ are constructed over $\mathcal{T}^{h}$ the corresponding shape functions $n_{a}$ on $\gamma\left(\mathcal{T}^{h}\right)$ may not be polynomials. They are definitively not polynomials when the map defined by $q(\eta)$ in (7) is adopted. Figure B.1 showcases the transformation of the shape functions $N_{a}$ to $n_{a}$ through the action of the map $\gamma(\hat{x})=\hat{x}^{2}$.

Further, it is easy to see that

$$
\boldsymbol{u}^{h}=\hat{\boldsymbol{u}}^{h} \circ \boldsymbol{\gamma}^{-1}=N_{a} \circ \boldsymbol{\gamma}^{-1} \boldsymbol{u}_{a}=n_{a} \boldsymbol{u}_{a},
$$

and similarly $\boldsymbol{w}^{h}=n_{a} \boldsymbol{w}_{a}$.

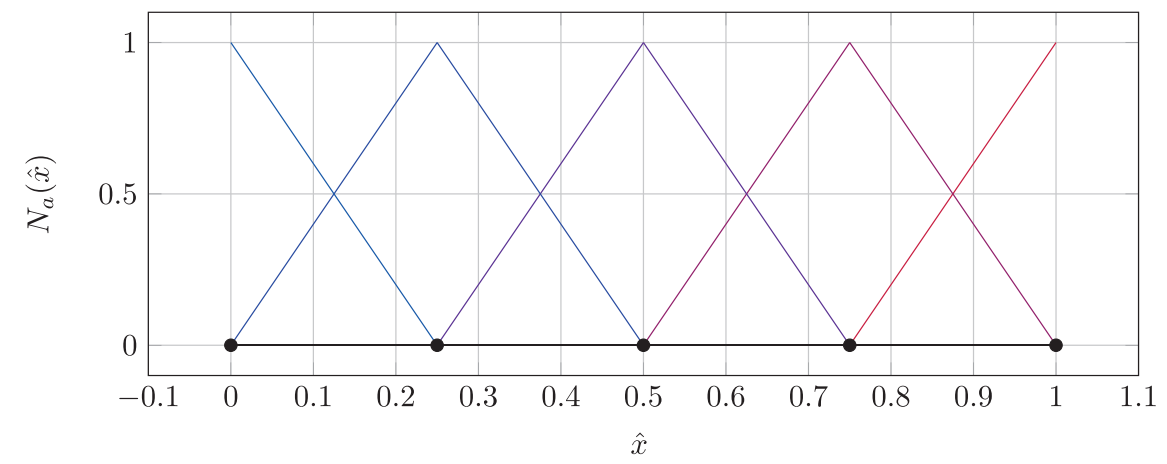

(a) A traditional set of affine shape functions constructed on a uniform discretization of the parametric domain

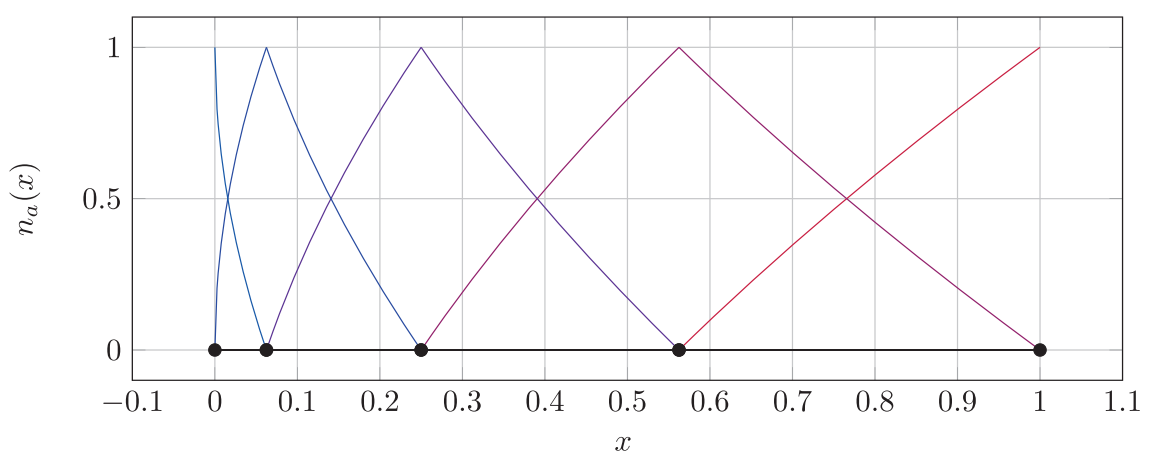

(b) Basis functions on the physical domain obtained by composing the set of affine shape functions in (a) with the mapping $\gamma(\hat{x})=\hat{x}^{2}$.

Figure B.1. Constructing a traditional affine finite element space $\left\{N_{a}\right\}_{a}$ on a uniform discretization $\mathcal{T}^{h}=$ $\bigcup_{i=0}^{4}\{[i / 4,(i+1) / 4]\}$ of the parametric domain $\hat{\Omega}=[0,1]$. Under the mapping $\gamma(\hat{x})=\hat{x}^{2}$ results in a set of shape functions $\left\{n_{a}\right\}_{a}$, no longer affine, on a subdivision of $\Omega$ given by $\gamma\left(\mathcal{T}^{h}\right)=\bigcup_{i=0}^{4}\{[\gamma((i / 4)), \gamma((i+$ 1)/4)]\}. (a) A traditional set of affine shape functions constructed on a uniform discretization of the parametric domain; (b) basis functions on the physical domain obtained by composing the set of affine shape functions in (a) with the mapping $\gamma(\hat{x})=\hat{x}^{2}$. 
Then (13) may be written as follows: find $\boldsymbol{u}_{a}$ such that

$$
\boldsymbol{w}_{a} \cdot \boldsymbol{k}_{a b} \boldsymbol{u}_{b}=\boldsymbol{w}_{a} \cdot \boldsymbol{f}_{a}, \quad \forall \boldsymbol{w}_{a},
$$

or find $\boldsymbol{U}$ such that

$$
\boldsymbol{K} \boldsymbol{U}=\boldsymbol{F}
$$

where

$$
\{\boldsymbol{K}\}_{a b}=\boldsymbol{k}_{a b}, \quad\{\boldsymbol{U}\}_{a}=\boldsymbol{u}_{a}, \quad\{\boldsymbol{F}\}_{a}=\boldsymbol{f}_{a}
$$

and

$$
\begin{aligned}
{\left[\boldsymbol{k}_{a b}\right]_{i s} } & =\sum_{K \in \mathcal{T}^{h}} \int_{K} \frac{\partial N_{a}}{\partial \hat{x}_{s}}[\mathbb{M}]_{i k s l} \frac{\partial N_{b}}{\partial \hat{x}_{l}} d K, \\
{\left[\boldsymbol{f}_{a}\right]_{i} } & =\sum_{K \in \mathcal{T}^{h}} \int_{K}[\hat{\boldsymbol{b}}]_{i} N_{a} j d K+\sum_{E \in \mathcal{E}_{\tau}} \int_{E}\left([\hat{\boldsymbol{t}}]_{i}\left\|(\nabla \boldsymbol{\gamma})^{-\top} \cdot \boldsymbol{n}\right\| j\right) \circ \mathfrak{p} d \hat{\Gamma}
\end{aligned}
$$

where $\mathcal{E}_{\tau}$ denotes the set of element edges on $\partial_{\tau} \Omega^{h},[\bullet]_{a b}$ and $[\bullet]_{a}$ denote the sub-tensors and vectors, respectively, associated with the degree of freedom $a$ and $b$, and $[\bullet]_{i s}=\bullet: \mathbf{e}_{i} \otimes \mathbf{e}_{s}$ and similarly $[\bullet]_{i s k l}=\bullet: \mathbf{e}_{i} \otimes \mathbf{e}_{s} \otimes \mathbf{e}_{k} \otimes \mathbf{e}_{l}$ denote the tensor components in the orthonormal basis associated with the Cartesian coordinates $x_{i}, \hat{x}_{i}$ (cf. Figure 3 ). The aforementioned integrals can then be approximated over each element $K$ using standard quadrature rules as commonly carried out in FEM.

Effectively, the only pecularities of this approach are that the coefficients of $\mathbb{M}$ will depend on space and further that $\mathbb{M}$ may not necessarily be symmetric. These peculiarities are easily handled in any commercial or open-source finite element code that allows for user-defined coefficients. Thus, the method is easy to implement, without the need to introduce special shape functions, additional quadrature rules, or particular mesh gradations.

\section{APPENDIX C: FORMULATION OF POISSON'S EQUATION OVER THE PARAMETRIC DOMAIN}

We construct here the weak form over the parametric domain of Poisson's equation for a scalar field, in a similar manner as presented in Section 3.

Formulation in the physical domain. We consider the problem of finding $u: \Omega \rightarrow \mathbb{R}$ such that

$$
\begin{aligned}
\Delta u+b & =0, & & \text { in } \Omega, \\
u & =g, & & \text { on } \partial_{d} \Omega, \\
\nabla u \cdot \boldsymbol{n} & =t, & & \text { on } \partial_{\tau} \Omega .
\end{aligned}
$$

Here, $b, g$, and $t$ are smooth enough scalar fields over the domains where they are evaluated.

The weak form of this problem reads: Find $u \in \mathcal{V}$ such that

$$
a(u, v)=F(w), \quad \forall w \in \mathcal{W},
$$

where

$$
\begin{aligned}
a(u, w) & :=\int_{\Omega} \nabla u \cdot \nabla w d \Omega, \\
F(w) & :=\int_{\Omega} b w d \Omega+\int_{\partial_{\tau} \Omega} t w d \Gamma
\end{aligned}
$$

and

$$
\begin{gathered}
\mathcal{V}=\left\{u \in H^{1}(\Omega) \mid u=g \text { on } \partial_{d} \Omega\right\}, \\
\mathcal{W}=\left\{w \in H^{1}(\Omega) \mid w=0 \text { on } \partial_{d} \Omega\right\} .
\end{gathered}
$$


Weak form over the parametric domain. With $\hat{\mathcal{V}}=\mathcal{V} \circ \gamma$ and $\hat{\mathcal{W}}=\mathcal{W} \circ \gamma$, the equivalent formulation is given by the following: Find $\hat{u} \in \hat{\mathcal{V}}$ such that

$$
a_{\gamma}(\hat{u}, \hat{w})=\hat{F}(\hat{w}), \quad \forall \hat{w} \in \hat{\mathcal{W}},
$$

where

$$
\begin{aligned}
& a_{\gamma}(\hat{u}, \hat{w}):=\int_{\hat{\Omega}} \nabla \hat{u} \cdot \boldsymbol{M} \cdot \nabla \hat{w} d \hat{\Omega},
\end{aligned}
$$

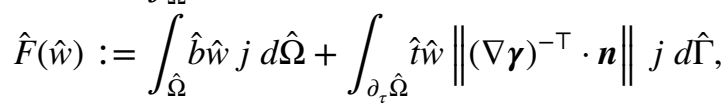

and

$$
\boldsymbol{M}=j(\nabla \boldsymbol{\gamma})^{-1} \cdot(\nabla \boldsymbol{\gamma})^{-\top} .
$$

Galerkin's approximation over the parametric domain. Let $\mathcal{T}^{h}$ denote a subdivision of $\hat{\Omega}$ as stated in Section 3.2. Consider finite dimensional approximations of $\hat{\mathcal{V}}$ and $\hat{\mathcal{W}}$ given by

$$
\begin{aligned}
& \hat{\mathcal{V}}^{h, p}=\left\{\hat{u}^{h} \in H^{1}(\hat{\Omega})\left|\hat{u}^{h}\right|_{K} \in P^{p}(K), \hat{u}^{h}=\hat{\mathcal{I}}^{h}(g \circ \gamma) \text { on } \partial_{d} \hat{\Omega}\right\}, \\
& \hat{\mathcal{W}}^{h, p}=\left\{\hat{w}^{h} \in H^{1}(\hat{\Omega})\left|\hat{w}^{h}\right|_{K} \in P^{p}(K), \hat{w}^{h}=0 \text { on } \partial_{d} \hat{\Omega}^{h}\right\},
\end{aligned}
$$

respectively. The Galerkin form of the problem statement then reads: Find $\hat{u}^{h} \in \hat{\mathcal{V}}^{h, p}$ such that

$$
a_{\gamma}\left(\hat{u}^{h}, \hat{w}^{h}\right)=\hat{F}\left(\hat{w}^{h}\right), \quad \forall \hat{w}^{h} \in \hat{\mathcal{W}}^{h, p} .
$$

\section{ACKNOWLEDGEMENTS}

This work was supported by the National Natural Science Foundation of China (11402146), Young 1000 Talent Program of China (Y.S.), National Science Foundation (CMMI-1301396), and Army Research Grant (W911NF-07-2-0027).

\section{REFERENCES}

1. Fix GJ, Gulati S, Wakoff GI. On the use of singular functions with finite element approximations. Journal of Computational Physics 1973; 13(2):209-228.

2. Belytschko T, Black T. Elastic crack growth in finite elements with minimal remeshing. International Journal for Numerical Methods in Engineering 1999; 45(5):601-620.

3. Moës N, Dolbow J, Belytschko T. A finite element method for crack growth without remeshing. International Journal for Numerical Methods in Engineering 1999; 46(1):131-150.

4. Melenk JM, Babuška I. The partition of unity finite element method: Basic theory and applications. Computer Methods in Applied Mechanics and Engineering 1996; 139(1-4):289-314.

5. Duarte CA, Oden JT. An h-p adaptive method using clouds. Computer Methods in Applied Mechanics and Engineering 1996; 139(1-4):237-262.

6. Liu XY, Xiao QZ, Karihaloo BL. XFEM for direct evaluation of mixed mode SIFs in homogeneous and bi materials. International Journal for Numerical Methods in Engineering 2004; 59(8):1103-1118.

7. Laborde P, Pommier J, Renard Y, Salaün M. High-order extended finite element method for cracked domains. International Journal for Numerical Methods in Engineering 2005; 64(3):354-381.

8. Béchet E, Minnebo H, Moës N, Burgardt B. Improved implementation and robustness study of the x-fem for stress analysis around cracks. International Journal for Numerical Methods in Engineering 2005; 64(8):1033-1056.

9. Shen Y, Lew AJ. An optimally convergent discontinuous Galerkin-based extended finite element method for fracture mechanics. International Journal for Numerical Methods in Engineering 2010; 82(6): 716-755.

10. Belytschko T, Gracie R, Ventura G. A review of extended/generalized finite element methods for material modeling. Modelling and Simulation in Materials Science and Engineering 2009; 17(4):043001, 24.

11. Yazid A, Abdelkader N, Abdelmadjid H. A state-of-the-art review of the x-fem for computational fracture mechanics. Applied Mathematical Modelling 2009; 33(12):4269-4282.

12. Henshell RD, Shaw KG. Crack tip finite elements are unnecessary. International Journal for Numerical Methods in Engineering 1975; 9(3):495-507. 
13. Barsoum RS. On the use of isoparametric finite elements in linear fracture mechanics. International Journal for Numerical Methods in Engineering 1976; 10(1):25-37.

14. Barsoum RS. Triangular quarter-point elements as elastic and perfectly-plastic crack tip elements. International Journal for Numerical Methods in Engineering 1977; 11(1):85-98.

15. Tracey DM. Finite elements for determination of crack tip elastic stress intensity factors. Engineering Fracture Mechanics 1971; 3(3):255-265.

16. Babuška I, Kellogg RB, Pitkäranta J, Babuska I, Kellogg RB, Pitkranta J. Direct and inverse error estimates for finite elements with mesh refinements. Numerische Mathematik 1979; 33(4):447-471.

17. Thatcher RW. The use of infinite grid refinements at singularities in the solution of Laplace's equation. Numerische Mathematik 1976; 25(2):163-178.

18. Karihaloo BL, Xiao QZ. Accurate determination of the coefficients of elastic crack tip asymptotic field by a hybrid crack element with p-adaptivity. Engineering Fracture Mechanics 2001; 68(15):1609-1630.

19. Tsamasphyros G, Giannakopoulos AE. The mapped elements for the solution of cracked bodies. Computer Methods in Applied Mechanics and Engineering 1985; 49(3):331-342.

20. Tsamasphyros G. Singular element construction using a mapping technique. International Journal for Numerical Methods in Engineering 1987; 24(7):1305-1316.

21. Babuška I, Oh H-S, Babugka I, Oh H-S. The p-version of the finite element method for domains with corners and for infinite domains. Numerical Methods for Partial Differential Equations 1990; 6(4):371-392.

22. Babuska I, Szabo BA, Katz IN. The p-version of the finite element method. SIAM Journal on Numerical Analysis 1981; 18(3):515-545.

23. Gordon W, Hall C. Construction of curvilinear co-ordinate systems and applications to mesh generation. International Journal for Numerical Methods in Engineering 1973; 7:461-477.

24. Lucas T, Oh H. The method of auxiliary mapping for the finite element solutions of elliptic problems containing singularities. Journal of Computational Physics 1993; 108(2):327-342.

25. Oh H-S, Babuška I, Babu ska I. The method of auxiliary mapping for the finite element solutions of elasticity problems containing singularities. Journal of Computational Physics 1995; 121(2):193-212.

26. Lee S-J, Oh H-S, Yun J-H. Extension of the method of auxiliary mapping for three-dimensional elliptic boundary value problems. International Journal for Numerical Methods in Engineering 2001; 50(5):1103-1129.

27. Jeong JW, Oh H-S, Kang S, Kim H. Mapping techniques for isogeometric analysis of elliptic boundary value problems containing singuliesarit. Computer Methods in Applied Mechanics and Engineering 2013; 254:334-352.

28. Grisvard P. Elliptic problems in nonsmooth domains. Society for Industrial and Applied Mathematics, 2011.

29. Grisvard P. Singularités en Élasticité. Archive for Rational Mechanics and Analysis 1989; 107:157-180.

30. Williams ML. Stress singularities resulting from various boundary conditions in angular corners of plate in extension. Journal of Applied Mechanics 1952; 19:526-528.

31. Williams ML. On the stress distribution at the base of a stationary crack. Journal of Applied Mechanics 1957; 24:109-158.

32. Brenner SC, Scott LR. The mathematical theory of finite element methods (2nd edn.) Springer: New York, 2002.

33. Muskhelishvili NI (ed.) Some Basic Problems of the Mathematical Theory of Elasticity: Fundamental Equations, Plane Theory of Elasticity, Torsion, and Bending (Translated from Russian) (2nd edn.) Noordhoff International Publishing: Leyden, The Netherlands, 1977.

34. Rossum G. Python reference manual, CWI (Centre for Mathematics and Computer Science), Amsterdam, The Netherlands, The Netherlands, 1995.

35. Rangarajan R, Chiaramonte MM, Hunsweck MJ, Shen Y, Lew AJ. Simulating curvilinear crack propagation in two dimensions with universal meshes. International Journal for Numerical Methods in Engineering 2015; 102(3-4):632-670.

36. Chiaramonte MM, Shen Y, Keer LM, Lew AJ. Computing stress intensity factors for curvilinear cracks. International Journal for Numerical Methods in Engineering 2015; 104(4):260-296.

37. Strang G, Fix GJ. An Analysis of the Finite Element Method. Prentice-Hall: Englewood Cliffs, New Jersey, 1973.

38. Suo Z. Mechanics of interface fracture. Ph.D. Thesis, 1989.

39. Sukumar N, Huang ZY, Prévost J-H, Suo Z. Partition of unity enrichment for bimaterial interface cracks. International Journal for Numerical Methods in Engineering 2004; 59(8):1075-1102.

40. Wloka J. Partial Differential Equations. Cambridge University Press: Cambridge, 1987.

41. Constantine GM, Savits TH. A multivariate Faa di Bruno formula with applications. Transactions of the American Mathematical Society 1996; 348(2):503-520.

42. Adams RA, Fournier JJF. Sovolev Spaces (2nd edn.) Academic Press: Amsterdam, 2003. 